\title{
Dinâmica de vórtices em superfícies com aplicações ao problema de dois vórtices no toro plano
}

\author{
Humberto Henrique de Barros Viglioni
}

TESE APRESENTADA

$\mathrm{AO}$

Instituto De Matemática e Estatística

DA

Universidade DE SÃo PaUlo

PARA

OBTENÇÃO DO TÍTULO

$\mathrm{DE}$

DOUTOR EM CIÊNCIAS

\author{
Programa: Matemática Aplicada \\ Orientador: Prof. Dr. Clodoaldo Grotta Ragazzo
}

Durante o desenvolvimento deste trabalho o autor recebeu auxílio financeiro da CNPq

São Paulo, Maio de 2013 


\section{Dinâmica de vórtices em superfícies com aplicações ao problema de dois vórtices no toro plano}

Esta versão da tese contém as correções e alterações sugeridas pela Comissão Julgadora durante a defesa da versão original do trabalho, realizada em 15/05/2013. Uma cópia da versão original está disponível no Instituto de Matemática e Estatística da Universidade de São Paulo.

Comissão Julgadora:

- Prof. Dr. Clodoaldo Grotta Ragazzo (orientador) - IME-USP

- Profa. Dra. Helena Maria Ávila de Castro - IME-USP

- Prof. Dr. Hildeberto Cabral - UFPE

- Prof. Dr. Jair Koiller - FGV

- Profa. Dra. Stefanella Boatto - UFRJ 


\section{Dedicatória}

Dedico este trabalho à minha mãe Tereza Longine de Barros Viglioni (in memorian) e à minha avó Maria José Guglielmelli Viglioni (in memorian). 


\section{Agradecimentos}

Quero registrar meu grande agradecimento a meu orientador, professor Clodoaldo Grotta Ragazzo, cujo inestimável apoio e incentivo tornou possível a conclusão desta tese. Em nossas longas conversas sobre o projeto, pude entender a importância da compreensão física do modelo de vórtices, sobretudo no que diz respeito à abordagem adequada para deduzir as equações de movimento. Seus conselhos, sugestões e ideias, adicionados de um fino senso crítico, foram de grande valor para o desenvolvimento e conclusão deste trabalho. Aos professores Jair koiller e Stefanella Boatto, agradeço muitíssimo pelas sugestões de problemas interessantes e pelas informações que auxiliaram de forma decisiva a elaboração desta tese. De fato, não posso deixar de registrar a importância do artigo Vortices on Closed Manifolds[BK08], cujas ideias e técnicas forneceram elementos de grande relevância para a compreensão e formulação geral do problema da dinâmica de vórtices. Ao professor Hildeberto Cabral, meus agradecimentos pelas sugestões e minuciosas observações que muito contribuíram para a qualidade do presente texto.

Agradeço à professora Helena de Castro e ao professor Gláucio Terra pelo apoio na difícil etapa de adaptação no início de meu doutorado. Também registro um especial agradecimento ao professor Jorge Sotomayor Tello pelo apoio e pelo conhecimento transmitido de forma sempre inteligente e estimulante, o que bastante contribuiu para minha motivação em estudar matemática.

Agradeço também a todos meus colegas e amigos, especialmente a meu amigo Michel Henrique Pereira que me recebeu em minha chegada a São Paulo, a Getúlio Bulhões e aos demais colegas do IME, bem como a todo pessoal do Departamento de Matemática da Universidade Federal de Sergipe.

Em especial, agradeço a meus pais Gorki e Rosa, pelo carinho, pela amizade e pelo inestimável apoio, que durante uma longa e boa convivência me auxiliaram a trilhar meu caminho sempre enfrentando os desafios necessários. A meus irmãos Leonardo, Alexei, George, Renata e Amanda, agradeço pela presença de vocês em minha vida. A Débora Lopes, mais que uma amiga, agradeço muito pelo apoio e compreensão, fundamentais para que eu pudesse conciliar minhas responsabilidades como pai, meus compromissos familiares e o desenvolvimento e conclusão desta tese. À minha pequena flor, Ana Rosa, fonte de alegria e ânimo, obrigado por ter me mostrado, no silêncio de sua inocência, que, na vida, é dividindo que se multiplica. 


\section{Resumo}

VIGLIONI, H. H. B. Dinâmica de vórtices em superfícies com aplicações ao problema de dois vórtices no toro plano. 2013. 75 f. Tese (Doutorado) - Instituto de Matemática e Estatística, Universidade de São Paulo, São Paulo, 2013.

Este trabalho apresenta uma dedução das equações para a dinâmica de vórtices em superfícies utilizando argumentos físicos e balanço de momento, obtendo o resultado já conhecido devido a Boatto \& Koiller [BK08] e Hally [Hal]. Na primeira parte, elaboramos uma releitura da contribuição de diversos pesquisadores incluindo, além dos já citados, o trabalho de Marchioro e Pulvirenti[MP93] sobre a propriedade de localização para a equação de Euler e também a importante contribuição de Flucher e Gustaffson[FG97, Gus79] no que diz respeito à determinação da função de Green e função de Robin hidrodinâmicas em domínios do plano. Na segunda parte revisamos o problema da dinâmica de um traçador passivo induzida por um vórtice no disco unitário e estendemos para o caso com vorticidade de fundo constante. Por fim, analisamos a dinâmica de dois vórtices no toro plano, a qual se reduz ao estudo da dinâmica do centro de vorticidade com hamiltoniana dada pela função de Green. É feita uma descrição das bifurcações das curvas de níveis desta hamiltoniana com respeito a variações do parâmetro modular. Mostramos que o campo hamiltoniano em questão é preservado por biholomorfismos e, portanto, o espaço dos parâmetros pode ser reduzido ao espaço de Moduli do toro plano. Mudanças dentro de uma mesma classe de equivalência por biholomorfismos podem alterar apenas a classe de homotopia das curvas de nível.

Palavras-chave: vórtices, superfícies, função de Green, função de Robin, potencial complexo, potencial velocidade, função de corrente, equação de Euler, toro, disco unitário, superfície de Riemann, Moduli, Teichmüller. 


\section{Abstract}

VIGLIONI, H. H. B. Vortex dynamics on surfaces with applications to the problem of two vortices in a flat torus. 2013. 75 f. Tese (Doutorado) - Instituto de Matemática e Estatística, Universidade de São Paulo, São Paulo, 2013.

In this thesis the equations for the motion of vortices on Riemannian surfaces is studied. Using conservation of momentum and physical arguments, the classical equations of Hally[Hal] and Boatto \& Koiller[BK08] are recovered. Then the localization result for the Euler's equation with flat metric (Marchioro \& Pulvirenti[MP93]) and the determination of the Green's and Robin's functions on plane domains are revisited in the context of Riemannian surfaces. On a second part of the thesis two examples are analyzed. At first the dynamics of a passive tracer in the unit disk on the flat plane with constant background vorticity. At second the dynamics of two vortices on flat tori. This last system is integrable. The dynamics is determined by the level sets of the Green's function which depends on the modular parameter of the torus. The full bifurcation diagram of the system as a function of the module parameter is determined.

Keywords: vortex, surfaces, Green's function, Robin's function, complex potential, velocity potential, stream function, Euler's equation, torus, unit disc, Riemann surfaces, Moduli, Teichmüller. 


\section{Sumário}

Lista de Símbolos $\quad$ xi

Lista de Figuras $\quad$ xiii

1 Introdução $\quad 1$

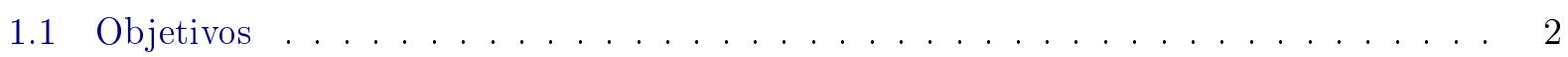

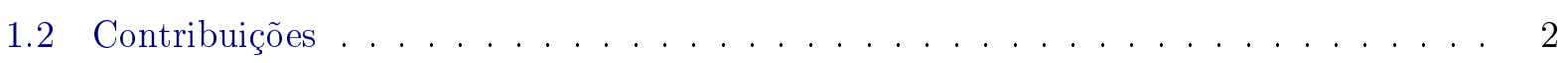

1.3 Organização do Trabalho . . . . . . . . . . . . . . . . . . . . . 3

2 Abordagem de Friedrichs $\quad 5$

2.1 Determinação da força exercida por um fluido . . . . . . . . . . . . . . 5

2.2 Equações de Movimento . . . . . . . . . . . . . . . . . . . . . . 6

3 Dinâmica de Vórtices: Abordagem Alternativa $\quad 11$

3.1 Vórtices em domínios do plano . . . . . . . . . . . . . . . . 12

3.2 Vórtices em domínios do plano munidos de uma métrica conforme . . . . . . . . . . . 15

3.3 Dinâmica de vórtices em superfícies fechadas . . . . . . . . . . . . . . . . 25

4 Considerações Adicionais Sobre Dinâmica de Vórtices $\quad 29$

4.1 Determinação da Função de Corrente . . . . . . . . . . . . . . . . . . . . . . . . 29

4.2 Propriedade de Localização . . . . . . . . . . . . . . . . . . . . 31

4.2.1 Sobre a construção de uma sequência delta preservada pela equação de Euler 34

$\begin{array}{lll}5 & \text { Exemplos } & \mathbf{3 7}\end{array}$

5.1 Disco Unitário . . . . . . . . . . . . . . . . . . . . . . 37

5.1 .1 Determinação da função de corrente . . . . . . . . . . . . . . . . 37

5.1 .2 Equações de Movimento . . . . . . . . . . . . . . . . . . . . . . 38

5.1 .3 Dinâmica do traçador passivo . . . . . . . . . . . . . . . . 40

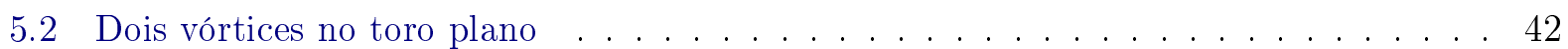

5.2 .1 Determinação da função de corrente no toro plano . . . . . . . . . . . . . . 42

5.2.2 Bifurcações das curvas de nível da função de Green no toro plano . . . . . . . . 44

5.2.3 Ação do grupo modular na estrutura das curvas de nível da função de Green do toro plano . . . . . . . . . . . . . . . . . . . . . 59

6 Conclusões $\quad 71$

6.1 Considerações Finais . . . . . . . . . . . . . . . . . . . . 71 
6.2 Sugestões para Pesquisas Futuras . . . . . . . . . . . . . . . . . . . 72

Referências Bibliográficas 73

Índice Remissivo 


\section{Lista de Símbolos}

v Campo de velocidades

$\omega$ Vorticidade

$\Gamma \quad$ Intensidade de um vórtice

$J$ Matriz Simplética

$\psi$ Função de corrente

$\phi$ Potencial de velocidade

$F \quad$ Potencial complexo

$\mathcal{F}$ Força 


\section{Lista de Figuras}

5.1 As curvas em vermelho (versão eletrônica) nos gráficos dos níveis de $H^{\prime \prime}$ representam as separatrizes heteroclínicas conectando as singularidades hiperbólicas localizados no bordo do disco. Nos gráficos das trajetórias, a do vórtice é representada pelo círculo azul (versão eletrônica) e a do traçador passivo pela curva vermelha (versão eletrônica), sendo a posição inicial do vórtice dada por $\left(\rho_{0}, 0\right)$ enquanto que a do traçador passivo é a mesma em todos os gráficos: $(0.3,-0.5) . \ldots \ldots$. . . . . . 40

5.2 Trajetórias do traçador passivo com variação negativa do parâmetro $\lambda$. Posição inicial do vórtice: $(0.5,0)$; posição inicial do traçador passivo: $(0.3,-0.5)$. . . . . . . . . . 41

5.3 Trajetórias do traçador passivo com variação positiva do parâmetro $\lambda$. Posição inicial do vórtice: $(0.5,0)$; posição inicial do traçador passivo: $(0.3,-0.5)$. . . . . . . . . 41

5.4 Trajetórias do traçador passivo em uma vizinhança do valor crítico. Posição inicial do vórtice: $(0.5,0)$; posição inicial do traçador passivo: $(0.3,-0.5)$. . . . . . . . . 41

5.5 O domínio fundamental para um toro plano é um paralelogramo $\mathcal{P}$ cujos lados opostos são identificados preservando a orientação. . . . . . . . . . . . . . . . . 42

5.6 a) conjunto de degenerescência de $\left.w_{1} ; b\right)$ conjunto de degenerecência de $w_{2} ; c$ ) conjunto de degenerescência de $\left.w_{3} ; d\right)$ representação do diagrama de bifurcação dos

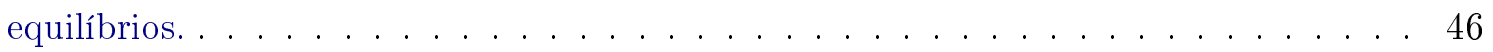

5.7 Retratos de fase correspondentes aos pontos $R 1, B 1, R 2, B 2, R 3$ e $R 5 \ldots \ldots$. . . . 47

5.8 Retratos de fase correspondentes aos pontos $B 4, R 4, B 6, R 6, B 5$ e $R 7$. . . . . . 48

5.9 Representação dos pontos para os quais ocorre conexão entre: $a) w_{1}$ e $\left.w_{2} ; b\right) w_{1}$ e $w_{3}$. 49

5.10 a) gráfico do conjunto $C_{23}$, o qual representa os pontos para os quais ocorre conexão entre os meio-períodos $w_{2}$ e $\left.w_{3} ; b\right)$ representação do diagrama de bifurcação das conexões, onde assinala-se as regiões selecionadas para análise do retrato de fase. . . 50

5.11 Retratos de fase correspondentes aos pontos $1,11,112,12,123$ e 13 . . . . . . . . . 51

5.12 Retratos de fase dos pontos $134,14,145,15,156,16$ e $161 \ldots \ldots \ldots$. . . . . . . . 52

5.13 Retratos de fase dos pontos $2,21,212$ e $22 \ldots \ldots \ldots \ldots \ldots \ldots$

5.14 Retratos de fase dos pontos $223,23,234,24,245,25,256,26$ e 261 . . . . . . . . . 54

5.15 Retratos de fase dos pontos $3,361,31,312$ e $32 \ldots \ldots \ldots \ldots \ldots$

5.16 Retratos de fase dos pontos $323,33,334,34,345,35,356$ e $36 \ldots \ldots$. . . . . . . 56

5.17 Retratos de fase dos pontos $4,461,41,412$ e $42 \ldots \ldots \ldots \ldots$. . . . . . . 57

5.18 Retratos de fase dos pontos $423,43,434,44,445,45,456$ e $46 \ldots \ldots$. . . . . . . . 58

5.19 Reticulado no plano . . . . . . . . . . . . . . . . . . . . . 59

5.20 Ilustração de duas bases distintas para um mesmo reticulado, apresentando os domínios fundamentais correspondentes. . . . . . . . . . . . . . . . 6 60 
5.21 Domínio fundamental para o parâmetro modular $\tau$. . . . . . . . . . . . . 60

5.22 Toros biholomorficamente equivalentes . . . . . . . . . . . . . . . . . . 61

5.23 a) retrato de fase do sistema correspondente ao ponto 234 e $b$ ) representação de suas conexões homoclínicas e heteroclínicas . . . . . . . . . . . . . . . . . . 63

5.24 Representação das separatrizes de $G(z ; \tau)$ e do reticulado $\Lambda(\tau)$ com geradores $g_{1}=1$ e $g_{2}=\tau$ onde: $\left.a\right) \tau=0.52+0.25 i$ e $\left.b\right) \tau=\tau_{0}-1=-0.48+0.25 i \quad$. . . . . . . . . 64

5.25 Representação das separatrizes de $G(z ; \tau)$ e do reticulado $\Lambda(\tau)$ com geradores $g_{1}=1$ e $g_{2}=\tau$ onde: $\left.a\right) \tau=\tau_{2}$ e $\left.b\right) \tau=\tau_{3}$. . . . . . . . . . . . . . . . . . . 64

5.26 a) representação das separatrizes de $G\left(z ; w_{1}, w_{2}\right)$ e do reticulado $\Lambda\left(g_{1}, g_{2}\right)$ gerado pela base inicial $g_{1}=1=2 w_{1}$ e $\left.g_{2}=\tau_{0}=2 w_{2} ; b\right)$ representação das separatrizes de $G\left(z ; w_{1}, w_{2}\right)$ e do reticulado $\Lambda\left(g_{1}, g_{2}\right)$ correspondentes à base $B_{1}$ obtida a partir da base inicial pela ação de $M_{1}$. . . . . . . . . . . . . . . . . . . . . . . 65

$5.27 a)$ representação das separatrizes de $G\left(z ; w_{1}, w_{2}\right)$ e do reticulado $\Lambda\left(g_{1}, g_{2}\right)$ correspondentes à base $B_{2}$ obtida a partir da base $B_{1}$ pela ação de $\left.M_{2} ; b\right)$ representação das separatrizes de $G\left(z ; w_{1}, w_{2}\right)$ e do reticulado $\Lambda\left(g_{1}, g_{2}\right)$ correspondentes à base $B_{3}$ obtida a partir da base $B_{2}$ pela ação de $M_{3}$. . . . . . . . . . . . . . . . . . . . 65

5.28 Imagens de $\tau=\tau_{0}$ pela composição das ações de $M_{1}, M_{2}$ e $M_{3}$, nesta ordem. . . . . . 66

5.29 Diagramas de bifurcação dos equilíbrios (à esquerda) e das conexões (à direita), representados no domínio fundamental D . . . . . . . . . . . . . . . . . . . . 66

5.30 Retratos de fase correspondentes aos pontos de $P_{1}$ a $P_{8}$. . . . . . . . . . . . . . 67

5.31 Retratos de fase correspondentes aos pontos de $P_{9}$ a $P_{18}$. . . . . . . . . . . . . . . 68

5.32 Retratos de fase correspondentes aos pontos de $P_{19}$ a $P_{29}$. . . . . . . . . . . . . . 69

5.33 Gráfico da função $F_{12}$ em uma vizinhaça de $a=0.335$ para valores pequenos de $b$ : da esquerda para a direita, temos $b=0.01, b=0.001$ e $b=0.0001$. . . . . . . . . . 70

5.34 Curvas de nível da função $F_{12}$ na vizinhança de $(0.36,0)$. A figura à direita é uma ampliação da região em destaque na primeira figura. . . . . . . . . . . . . . . . . . 70 


\section{Capítulo 1}

\section{Introdução}

Vórtices hidrodinâmicos são singularidades do campo de velocidades de um fluido que surgem em virtude da concentração da vorticidade em pontos. Fisicamente, trata-se de um modelo ideal tal como a massa pontual. Como apresentado no texto de Marchioro \& Pulvirenti ([MP94]), um sistema de vórtices em um domínio $D \subset \mathbb{R}^{2}$ é matematicamente representado por um perfil de vorticidade inicial dado pela medida

$$
\omega(x) d x=\sum_{k=1}^{N} \Gamma_{k} \delta_{x_{k}}(d x),
$$

onde $\delta_{x_{k}}$ é a medida de Dirac concentrada no ponto $x_{k}$. Cada componente $\Gamma_{k} \delta_{x_{k}}(d x)$ é denominada um vórtice de intensidade $\Gamma_{k}$ localizado em $x_{k}$. Surge então uma questão interessante: considerando este perfil inicial de vorticidade, é possível definir uma dinâmica dos vórtices $\Gamma_{k} \delta_{x_{k}}(d x)$ de modo que a evolução temporal da vorticidade $\omega(x ; t) d x=\sum_{k=1}^{N} \Gamma_{k} \delta_{x_{k}(t)}(d x)$ seja matematicamente bem definida e deduzida a partir dos princípios físicos da mecânica dos fluidos ? Uma resposta a esta pergunta são as equações de Helmholtz-Kirchhoff que definem a dinâmica de vórtices no plano, cujo estudo remonta aos trabalhos pioneiros de Helmholtz (1858), Lord Kelvin (Sir William Thomson - 1867), Kirchhoff (1876) e J.J. Thompson (1884). As referências clássicas no assunto estabelecem o seguinte procedimento a fim de obter de equações de Helmholtz-Kirchhoff: cada vórtice no plano desloca-se com a velocidade induzida pelos demais. No caso de domínios do plano com bordo, a presença do obstáculo físico interfere na dinâmica dos vórtices de uma maneira natural, considerando que o campo de velocidades num tal domínio deve ser a sobreposição do campo gerado pelos vórtices com uma componente regular que é o gradiente simplético de uma função analítica no domínio de escoamento, cujo papel é corrigir a velocidade gerada pelos vórtices de modo que a resultante seja tangente ao bordo e satisfaça as prescrições das circulações em suas diversas componentes conexas [Lin41a, Lin41b, FG97]. Assim sendo, num domínio com bordo cada vórtice é transportado pelo campo de velocidades dos demais somado ao campo de velocidades induzido pela presença do bordo. Portanto, definindo a regularização do campo de velocidades na posição de cada vórtice removendo a componente singular devida ao próprio vórtice, temos que a evolução temporal do sistema de vórtices é definida de modo que, em um dado instante de tempo, a velocidade de cada vórtice é definida pela regularização do campo de velocidades em sua posição. No entanto, em casos mais gerais a situação é diferente, como nos mostra o resultado obtido por Hally [Hal] e Boatto \& Koiller [BK08] do qual conclui-se que, em superfícies fechadas, um único vórtice pode desenvolver uma dinâmica não trivial. Mais precisamente, em [BK08] demonstra-se que a hamiltoniana para a dinâmica de um vórtice em uma superfície fechada é dada em termos da função de Robin, definida como a regularização, na diagonal, da função de Green para o laplaciano na superfície. Veremos como este processo de regularização pode ser deduzido a partir do balanço de momento em uma vizinhaça do vórtice. Inicialmente, apresentamos uma abordagem deste problema devida a Friedrichs ([Fri66]), que utiliza-se de um referencial inercial adequado a fim de efetuar o balanço de momento linear em 
uma vizinhança do vórtice, possibilitando a determinação de sua equação de movimento. Embora esta abordagem nos permita determinar a dinâmica de vórtices livres e com massa no plano, ela apresenta duas grandes limitações. A primeira é que depende de uma mudança de referencial que não pode ser realizada em superfícies de maneira simples, dificultando a determinação da dinâmica em contextos mais gerais. Além disso, ela só é aplicavel a sistemas de vórtices com vorticidade de fundo nula, o que novamente restringe bastante a aplicabilidade do método. A fim de contornar estas duas limitações, apresentamos uma abordagem alternativa que permite a determinação das equações de movimento de um sistema de vórtices em contextos que envolvam curvatura e vorticidade de fundo constante não nula.

A concepção deste trabalho sofreu grande influência de diversos autores em diversas áreas, dentre os quais destacam-se:

- Dinâmica de vórtices: Hildeberto Cabral[CS00], Hally[Hal], Friedrichs[Fri66], Flucher e Gustaffson[FG97, Gus79], Marchioro e Pulvirenti[MP82, MP93], Boatto e Koiller[BK08], Crowdy[Cro10], P. Newton[New01]

- Equações diferenciais e Sistemas Hamiltonianos: Sotomayor[Sot79], Arnold[Arn92, Arn89]

- Mecânica dos fluidos: Marchioro e Pulvirenti[MP94], Majda e Bertozzi[MB02], Arnold e Khesin[AK98]

- Geometria: Struik[Str88], Manfredo[dC88]

- Teoria de Potencial, Função de Green e Superfícies de Riemann: Crowdy, Gustafsson, F. Klein[Kle63], Farkas e Kra[FK92], T. Aubin[Aub98], P. Li e L-F Tam[PL87], Lin e Wang [LW10]

\subsection{Objetivos}

Um dos objetivos deste trabalho é apresentar uma dedução das equações de movimento para um sistema de vórtices a partir dos princípios físicos da mecânica dos fluidos, elaborando um texto elementar e auto-contido que sirva como uma referência auxiliar ao estudo do tema. Outro objetivo é ilustrar o método com uma aplicação simples ao estudo da dinâmica de um traçador passivo induzido por um vórtice no disco unitário com vorticidade de fundo constante não nula. Por fim, visando abordar um caso de superfície fechada, descrevemos e analisamos a dinâmica de dois vórtices no toro plano e sua dependência do parâmetro modular.

\subsection{Contribuições}

As principais contribuições deste trabalho são as seguintes:

- Item 1 - Apresentar uma justificativa física razoável para o processo de regularização que resulta nas equações da dinâmica de vórtices, desenvolvendo uma abordagem que é aplicável a domínios com curvatura e com vorticidade de fundo constante não nula.

- Item 2 - Reunir, em um texto simples, as contribuições mais relevantes de diversos pesquisadores e que sejam importantes para uma boa compreensão do problema de vórtices em situações mais gerais.

- Item 3 - Analisar as bifurcações da dinâmica de dois vórtices no toro plano considerando variações no parâmetro modular.

- Item 4 - Identificar problemas matemáticos interessantes para a elaboração de temas para projetos de pesquisa na área. 


\subsection{Organização do Trabalho}

No Capítulo 2, apresentamos as ideias desenvolvidas por Friedrichs a fim de deduzir as equações da dinâmica de um sistema de vórtices no plano. Esta foi a principal referência na elaboração deste trabalho. De fato, na tentativa de contornar as obstruções matemáticas que impossibilitavam uma aplicação direta da abordagem de Friedrichs a domínios com curvatura e vorticidade de fundo constante não nula, desenvolvemos o método apresentado no Capítulo 3, cujo principal resultado são as equações da dinâmica de vórtices em superfícies. No Capítulo 4 abordamos o problema da determinação da função de corrente para um sistema de vórtices em um domínio do plano, bem como apresentamos o problema de valor inicial para a equação de Euler e os resultados de Marchioro e Pulvirenti sobre a propriedade de localização, utilizada na dedução das equações de movimento de um sistema de vórtices. No Capítulo 5, aplicamos a teoria desenvolvida ao estudo da dinâmica de um traçador passivo induzida por um vórtice no disco unitário, bem como ao estudo da dinâmica de dois vórtices no toro plano, finalizando com um estudo das bifurcações do problema de dois vórtices no toro plano com respeito a variações do parâmetro modular. Finalmente, no Capítulo 6 discutimos algumas conclusões obtidas neste trabalho, analisamos as vantagens e desvantagens do método proposto e apresentamos algumas perpectivas de pesquisa. 


\section{Capítulo 2}

\section{Abordagem de Friedrichs}

Neste capítulo apresentamos a dedução devida a Friedrichs das equações de Helmholtz-Kirchhoff que regem a dinâmica de um sistema de vórtices no plano. A principal ideia utilizada é observar o fluido a partir de um sistema de coordenadas que desloca-se com o vórtice, para então calcular o balanço de momento numa vizinhança da singularidade de modo a determinar a força que o fluido exerce sobre ela.

\subsection{Determinação da força exercida por um fluido}

Seja $D$ um domínio regular de $\mathbb{R}^{2}$ e $\mathbf{v}$ um campo de velocidades de classe $C^{2}$ definido em $D$ e que satisfaz a equação de Euler

$$
\begin{cases}\partial_{t} \mathbf{v}+(\mathbf{v} \cdot \nabla) \mathbf{v}=-\nabla p, & \text { em } D \times I \\ \operatorname{div}(\mathbf{v})=0, & \text { em } D \times I \\ \mathbf{v}(x, t) \cdot \hat{n}=0, & \text { em } \partial D\end{cases}
$$

onde $p=p(x, t)$ é a pressão escalar, $I$ é o intervalo $(-\epsilon, \epsilon) \operatorname{com} \epsilon>0$ e $\hat{n}$ é a normal exterior a $D$. Ao longo de todo texto, consideramos a densidade do fluido constante igual a 1 , de modo que a condição de divergente nulo para o campo de velocidades é a expressão para a equação da continuidade. A equação

$$
\partial_{t} \mathbf{v}+(\mathbf{v} \cdot \nabla) \mathbf{v}=-\nabla p
$$

é deduzida a partir da conservação do momento linear. De fato, como bem apresentado em diversos textos sobre o assunto ([MP94], [MB02]), o momento linear do fluido que, no instante $t$, ocupa a região $U_{t}=\Phi_{t}(U)$, onde $U \subset D$ é um aberto regular e $\Phi_{t}$ é o fluxo correspondente à formulação lagrangiana do problema em questão, é dado por

$$
\iint_{U_{t}} \mathbf{v}(x, t) d \mu .
$$

Pela segunda lei de Newton, a derivada da expressão acima é igual à força total agindo sobre $U_{t}$ e portanto, considerando a inexistência da ação de forças externas, obtemos

$$
\iint_{U_{t}} \partial_{t} \mathbf{v}+(\mathbf{v} \cdot \nabla) \mathbf{v} d \mu=-\int_{\partial U_{t}} p \hat{n} d s .
$$

Como o domínio $U_{t}$ é regular e o campo $\mathbf{v}$ de classe $C^{2}$, podemos aplicar o teorema de Gauss e reescrever o termo $\iint_{U_{t}}(\mathbf{v} \cdot \nabla) \mathbf{v} d \mu$ da equação $(2.2)$ como uma integral no bordo. De fato, a $i-$ ésima 
componente de $(\mathbf{v} \cdot \nabla) \mathbf{v}$ é $(\mathbf{v} \cdot \nabla) v^{i}$ e portanto, como div $(\mathbf{v})=0$, temos

$$
\iint_{U_{t}}(\mathbf{v} \cdot \nabla) v^{i} d \mu=\iint_{U_{t}}(\mathbf{v} \cdot \nabla) v^{i}+v^{i} d i v(\mathbf{v}) d \mu=\iint_{U_{t}} \operatorname{div}\left(v^{i} \mathbf{v}\right) d \mu=\iint_{\partial U_{t}} v^{i}(\mathbf{v}, \hat{n}) d s,
$$

donde segue-se

$$
\iint_{U_{t}}(\mathbf{v} \cdot \nabla) \mathbf{v} d \mu=\int_{\partial U_{t}} \mathbf{v}(\mathbf{v}, \hat{n}) d s
$$

onde a integral no bordo representa o fluxo de momento através da fronteira da região $U_{t}$. Com isto, temos a seguinte expressão para a conservação de momento:

$$
\iint_{U_{t}} \partial_{t} \mathbf{v} d \mu+\int_{\partial U_{t}} \mathbf{v}(\mathbf{v}, \hat{n}) d s=-\int_{\partial U_{t}} p \hat{n} d s
$$

Vejamos como esta expressão pode ser utilizada para determinar a força $\mathcal{F}$ que o fluxo de um fluido no plano exerce sobre o perfil de um objeto bidimensional estático, representado por um domínio regular $B \subset \mathbb{R}^{2}$. Sabe-se que

$$
\mathcal{F}=-\int_{\partial B} p \hat{n} d s
$$

e portanto, dado um aberto $U$ de bordo regular e que contenha o objeto $B$, fazendo $U_{t}=U \backslash B$ na equação (2.4) e considerando que $\int_{\partial B} \mathbf{v}(\mathbf{v}, \hat{n}) d s=0$ obtemos

$$
\mathcal{F}=-\iint_{U \backslash B} \partial_{t} \mathbf{v} d \mu-\int_{\partial U} \mathbf{v}(\mathbf{v}, \hat{n}) d s-\int_{\partial U} p \hat{n} d s,
$$

isto é, a força que o fluido exerce sobre o objeto $B$ dentro do domínio $U$ é a força total que o fluido exerce sobre $U$ menos a variação do momento em $U \backslash B$ somada com o fluxo de momento na fronteira de $U$. A equação (2.5) é o ponto de partida para o resultado apresentado por Friedrichs, que será desenvolvido na próxima seção.

\subsection{Equações de Movimento}

Utilizaremos os resultados obtidos na seção anterior para determinar a força que o fluido exerce sobre um vórtice. Consideramos o objeto $B \subset U$ degenerando-se em um ponto $\mathfrak{p}_{k}=\left(x_{k}, y_{k}\right) \in$ $U \subset \mathbb{R}^{2}$, em torno do qual o campo $\mathbf{v}$ tem circulação $\Gamma_{k}:=\int_{\partial U}(\mathbf{v}, \hat{t}) d s$. Introduzindo a função de corrente $\psi(\mathfrak{p}, t)$ como sendo a função que satisfaz

$$
\mathbf{v}=\nabla^{\perp} \psi=-J \nabla \psi
$$

onde $J=\left(\begin{array}{cc}0 & -1 \\ 1 & 0\end{array}\right)$ é a matriz simplética, temos que a vorticidade de $\mathbf{v}$ é a função

$$
\omega:=\nabla \times \mathbf{v}=\partial_{1} v_{2}-\partial_{2} v_{1}=-\Delta \psi
$$

A fim de apresentarmos a segunda lei de Bernoulli, consideremos a identidade

$$
(\mathbf{v} \cdot \nabla) \mathbf{v}=\frac{1}{2} \nabla\left(|\mathbf{v}|^{2}\right)+\omega \nabla \psi
$$

Nesta primeira parte do trabalho consideramos apenas fluidos irrotacionais, isto é, $\omega \equiv 0$. 
Portanto, a equação (2.6) escreve-se

$$
(\mathbf{v} \cdot \nabla) \mathbf{v}=\frac{1}{2} \nabla\left(|\mathbf{v}|^{2}\right)
$$

Além disso, o fato do fluido ser irrotacional implica que $0=\omega=-\Delta \psi$, isto é, $\psi$ é uma função harmônica e portanto existe uma função multivalorada $\phi(\mathfrak{p})$ denominada potencial de velocidade, definida como sendo a função harmônica conjugada de $\psi$, ou seja, a função complexa $F(z)=$ $\phi(z)+i \psi(z)$, denominada potencial complexo, é analítica. Assim sendo, verifica-se que $\mathbf{v}=\nabla \phi \mathrm{e}$ portanto, substituindo a relação (2.7) na equação de Euler, obtemos

$$
0=\partial_{t} \mathbf{v}+(\mathbf{v} \cdot \nabla) \mathbf{v}+\nabla p=\partial_{t} \nabla \phi+\frac{1}{2} \nabla\left(|\mathbf{v}|^{2}\right)+\nabla p=\nabla\left(\dot{\phi}+\frac{1}{2}|\mathbf{v}|^{2}+p\right),
$$

isto é, para fluidos irrotacionais vale que

$$
\dot{\phi}+\frac{1}{2}|\mathbf{v}|^{2}+p=\text { constante. }
$$

Esta é a segunda lei de Bernoulli. Decomponhamos o potencial de velocidade $\phi$ e o campo de velocidades $\mathbf{v}=\nabla \phi$ como soma de suas componentes singulares e regulares:

$$
\phi(\mathfrak{p})=\phi_{S}(\mathfrak{p})+\phi_{R}(\mathfrak{p}) \text { e } \mathbf{v}(\mathfrak{p})=\mathbf{v}_{S}(\mathfrak{p})+\mathbf{v}_{R}(\mathfrak{p}) .
$$

Os subíndices $S_{R}$ e ${ }_{R}$ denotam, respectivamente, as partes singular e regular do potencial de velocidade e do campo na vizinhança aberta $U$ do vórtice $\mathfrak{p}_{k}$. Explicitamente temos

$\phi_{S}(x, y)=\frac{\Gamma_{k}}{2 \pi} \operatorname{Arctan}\left(\frac{y-y_{k}}{x-x_{k}}\right)$ e $\mathbf{v}_{S}(x, y)=\frac{\Gamma_{k}}{2 \pi}\left(\frac{-y+y_{k}}{\left(x-x_{k}\right)^{2}+\left(y-y_{k}\right)^{2}}, \frac{x-x_{k}}{\left(x-x_{k}\right)^{2}+\left(y-y_{k}\right)^{2}}\right)$.

Note que como o fluido é irrotacional em $U \backslash\left\{\mathfrak{p}_{k}\right\}$, a parte singular do potencial de velocidade é uma função (multivalorada) harmônica em $U \backslash\left\{\mathfrak{p}_{k}\right\}$ e sua parte regular $\phi_{R}$ é harmônica em $U$, isto é

$$
\Delta \phi_{S}(x, y)=0 \text { em } U \backslash\left\{p_{k}\right\} \text { e } \Delta \phi_{R}(x, y)=0 \text { em } U
$$

Assim, utilizando os resultados apresentados na seção anterior temos que a força que o fluido exerce sobre o vórtice em $\mathfrak{p}_{k} \in U$ é

$$
\mathcal{F}=-\iint_{U \backslash\left\{\mathfrak{p}_{k}\right\}} \partial_{t} \mathbf{v} d \mu-\int_{\partial U} \mathbf{v}(\mathbf{v}, \hat{n}) d s-\int_{\partial U} p \hat{n} d s,
$$

Substituindo na equação acima a expressão da pressão dada pela segunda lei de Bernoulli (equação 2.8), encontramos

$$
\mathcal{F}\left(\mathfrak{p}_{k}\right)=-\iint_{U \backslash\left\{p_{k}\right\}} \partial_{t} \mathbf{v} d \mu+\frac{1}{2} \int_{\partial U}|\mathbf{v}|^{2} \hat{n}-2 \mathbf{v}(\mathbf{v}, \hat{n}) d s+\int_{\partial U} \dot{\phi} \hat{n} d s .
$$

A fim de calcularmos a expressão acima, observa-se o fluido de um referencial inercial ${ }^{1}$ que move-se com uma velocidade tal que o vórtice $p_{k}$ está parado no tempo inicial em relação a este sistema de referência. Desta forma, como $\dot{x}_{k}(0)=0$ e $\dot{y}_{k}(0)=0$ temos que $\partial_{t} \mathbf{v}_{S}=\mathbf{0}$ e $\dot{\phi}_{S}=0$ em $t=0$ e portanto, no instante inicial, vale $\partial_{t} \mathbf{v}=\partial_{t} \mathbf{v}_{R}$ e $\dot{\phi}=\dot{\phi}_{R}$. Então, aplicando o teorema de Gauss à

\footnotetext{
${ }^{1}$ Se o referencial não for inercial, devemos corrigir a equação de Euler de modo a considerar as forças fictícias que surgem no sistema. Esta é uma das dificuldades em utilizar esta abordagem para determinar a dinâmica de vórtices em superfícies.
} 
parte regular no instante $t=0$ encontramos

$$
-\iint_{U \backslash\left\{\mathfrak{p}_{k}\right\}} \partial_{t} \mathbf{v} d \mu+\int_{\partial U} \dot{\phi} \hat{n} d s=-\iint_{U \backslash\left\{\mathfrak{p}_{k}\right\}} \partial_{t} \mathbf{v}_{R} d \mu+\int_{\partial U} \dot{\phi}_{R} \hat{n} d s=-\iint_{U \backslash\left\{\mathfrak{p}_{k}\right\}} \nabla \dot{\phi}_{R} d \mu+\int_{\partial U} \dot{\phi}_{R} \hat{n} d s=0 .
$$

Substituindo este resultado na equação (2.9) obtemos que

$$
\mathcal{F}_{t=0}\left(\mathfrak{p}_{k}\right)=\frac{1}{2} \int_{\partial U}|\mathbf{v}|^{2} \hat{n}-2 \mathbf{v}(\mathbf{v}, \hat{n}) d s
$$

Utilizaremos notação complexa para identificar campos de vetores no plano com funções complexas e transformar a integral acima em uma integral complexa ${ }^{2}$. Para tanto, convém apresentar alguns simples resultados que nos permitirão traduzir nossas equações para notação complexa (Friedrichs [Fri66], pg 21). Inicialmente, dada uma curva parametrizada por comprimento de arco $\lambda(s)=\left(x_{1}(s), x_{2}(s)\right)$, seu vetor tangente é $\hat{t}:=\lambda^{\prime}=\left(\frac{d x_{1}}{d s}, \frac{d x_{2}}{d s}\right)$, que em notação complexa escrevese $\hat{t}=\frac{d x_{1}}{d s}+i \frac{d x_{2}}{d s}$. Considerando a convenção usual para escolha do vetor normal a uma curva, temos $\hat{n}:=-i \hat{t}=\frac{d x_{2}}{d s}-i \frac{d x_{1}}{d s}$, isto é,

$$
n_{1} d s=d x_{2}, \quad n_{2} d s=-d x_{1}
$$

e portanto $\hat{n} d s=-i d z$ e $\left(n_{1}-i n_{2}\right) d s=i d \bar{z}$. Além disso, temos

$$
(\mathbf{u}, \hat{n}) d s=u_{1} d x_{2}-u_{2} d x_{1}=\operatorname{Im}(\mathbf{u} d z)=\frac{i}{2}(\mathbf{u} d \bar{z}-\overline{\mathbf{u}} d z) .
$$

Utilizando estes fatos e reescrevendo a expressão da força sobre o vórtice em notação complexa obtemos

$$
\mathcal{F}_{t=0}=\frac{1}{2} \int_{\partial U}|\mathbf{v}|^{2}(-i) d z-\int_{\partial U} \mathbf{v} \frac{i}{2}(\mathbf{v} d \bar{z}-\overline{\mathbf{v}} d z)=-\frac{i}{2} \int_{\partial U} \mathbf{v}^{2} d \bar{z} .
$$

A expressão em notação complexa para o campo de velocidades é

$$
\mathbf{v}(z, t)=\frac{\mathbf{i} \Gamma_{k}}{2 \pi} \frac{\mathbf{z}-\mathbf{z}_{k}}{\left|z-z_{k}\right|^{2}}+\mathbf{v}_{R}(z, t),
$$

onde $\overline{\mathbf{v}}_{R}$ é uma função analítica numa vizinhança do $k$-ésimo vórtice. Sendo assim, temos

$$
\mathbf{v}^{2}=-\frac{\Gamma_{k}^{2}}{4 \pi^{2}} \frac{\left(z-z_{k}\right)^{2}}{\left|z-z_{k}\right|^{4}}+\mathbf{v}_{R}^{2}+\frac{i \Gamma_{k} \mathbf{v}_{R}}{\pi} \frac{z-z_{k}}{\left|z-z_{k}\right|^{2}}
$$

e portanto

$$
\overline{\mathbf{v}}^{2}=-\frac{\Gamma_{k}^{2}}{4 \pi^{2}} \frac{1}{\left(z-z_{k}\right)^{2}}+\overline{\mathbf{v}}_{R}^{2}-\frac{i \Gamma_{k} \overline{\mathbf{v}}_{R}}{\pi} \frac{1}{z-z_{k}} .
$$

Tomando o complexo conjugado da equação (2.11) e substituindo a identidade (2.12) na equação obtida encontramos

$$
\overline{\mathcal{F}}_{t=0}=\frac{i}{2} \int_{\partial U} \overline{\mathbf{v}}^{2} d z=-\frac{i}{2} \frac{i \Gamma_{k} \overline{\mathbf{v}}_{R}}{\pi} 2 \pi i=i \Gamma_{k} \overline{\mathbf{v}}_{R}\left(z_{k}\right) .
$$

Como o resultado acima foi escrito em termos de um referencial inercial que no instante inicial desloca-se com a velocidade $\dot{\mathbf{z}}_{k}$ do vórtice, no referencial original o resultado correto é

$$
\mathcal{F}_{t=0}=-i \Gamma_{k}\left(\mathbf{v}_{R}\left(z_{k}\right)-\dot{\mathbf{z}}_{k}\right) .
$$

\footnotetext{
${ }^{2}$ Por abuso de notação, utilizaremos indiscriminadamente o mesmo símbolo para representar um campo vetorial no plano e a função complexa correspondente a tal campo.
} 
Portanto, a equação de movimento para um vórtice livre, isto é, um vórtice sem massa, é

$$
\dot{\mathbf{z}}_{k}=\mathbf{v}_{R}\left(z_{k}\right),
$$

enquanto que um vórtice $\mathbf{z}_{k}$ com massa $m_{k}$ evolui segundo a equação

$$
m_{k} \ddot{\mathbf{z}}_{k}=-i \Gamma_{k}\left(\mathbf{v}_{R}\left(z_{k}\right)-\dot{\mathbf{z}}_{k}\right)
$$

Para o caso de um sistema de $n$-vórtices livres no plano temos que $\mathbf{v}_{R}=\sum_{l \neq k} \frac{i \Gamma_{l}}{2 \pi} \frac{\mathbf{z}-\mathbf{z}_{l}}{\left|z-z_{l}\right|^{2}}$ e portanto obtemos a EDO que rege a dinâmica de $n$-vórtices, conhecidas como equações de HelmholtzKirchhoff ${ }^{3}$ :

$$
\dot{\mathbf{z}}_{k}=\sum_{l \neq k} \frac{i \Gamma_{l}}{2 \pi} \frac{\mathbf{z}_{k}-\mathbf{z}_{l}}{\left|z_{k}-z_{l}\right|^{2}} .
$$

É importante observar que o fato de termos considerado o sistema num referencial em relação ao qual a velocidade inicial do vórtice é nula foi o que nos permitiu eliminar as componentes singulares de $\partial_{t} \mathbf{v}$ e de $\dot{\phi}$ na equação (2.10). Do contrário, não conseguiríamos determinar a equação da dinâmica do vórtice, uma vez que não seria possível trabalhar satisfatoriamente estas componentes singulares. Este fato impõe uma séria restrição à tentativa de generalizar o resultado obtido para superfícies. Outra limitação da abordagem acima apresentada é que ela não nos permite tratar a dinâmica de vórtices com vorticidade de fundo não nula. Tais situações podem surgir de modelos interessantes em domínios do plano bem como em superfícies compactas. A fim de contornar estas dificuldades e formular a dinâmica de vórtices num contexto mais geral, apresentamos, na próxima seção, um tratamento alternativo a este problema, que nos permite estender o resultado aqui apresentado.

\footnotetext{
${ }^{3}$ Esta é a versão complexa das equações de Helmholtz-Kirchhoff.
} 


\section{Capítulo 3}

\section{Dinâmica de Vórtices: Abordagem Alternativa}

No capítulo anterior apresentamos a seguinte expressão para a força que um fluido exerce sobre um corpo rígido $B$ nele mergulhado

$$
\mathcal{F}(B)=-\iint_{U \backslash B} \partial_{t} \mathbf{v} d \mu-\int_{\partial U} \mathbf{v}(\mathbf{v}, \hat{n}) d s-\int_{\partial U} p \hat{n} d s+\int_{\partial B} \mathbf{v}(\mathbf{v}, \hat{n}) d s,
$$

onde $U$ é uma vizinhança aberta de $B$, o qual, para facilitar os cálculos, assumimos ter o formato de um disco. Considerando a equação acima num instante de tempo no qual o objeto está parado, obtem-se que $\mathbf{v} \cdot \hat{n}=0$ em $\partial B$ e portanto a expressão para a força simplifica-se

$$
\mathcal{F}(B)=-\iint_{U \backslash B} \partial_{t} \mathbf{v} d \mu-\int_{\partial U} \mathbf{v}(\mathbf{v}, \hat{n}) d s-\int_{\partial U} p \hat{n} d s .
$$

No presente Capítulo, estamos interessados em determinar a força sobre um objeto que desloca-se com velocidade não nula na situação limite em que o mesmo degenera-se num ponto de massa $m$. Considerando que o deslocamento é rígido, a velocidade $\mathbf{v}$ do fluido em $\partial B$ pode ser decomposta como a soma de uma componente tangente ao bordo com a velocidade do objeto que, por sua vez, instantaneamente escreve-se como composição de uma rotação com velocidade angular $\Omega_{0} \mathrm{em}$ relação ao centro de massa com uma translação uniforme $\mathbf{u}_{0}$. Portanto, temos

$$
\lim _{B \rightarrow \mathfrak{p}} \int_{\partial B} \mathbf{v}(\mathbf{v}, \hat{n}) d s=\mathbf{0},
$$

donde segue-se que, considerando o limite $B \rightarrow \mathfrak{p}$, obtemos a seguinte expressão para a força

$$
\mathcal{F}(\mathfrak{p})=-\iint_{U \backslash \mathfrak{p}} \partial_{t} \mathbf{v} d \mu-\int_{\partial U} \mathbf{v}(\mathbf{v}, \hat{n}) d s-\int_{\partial U} p \hat{n} d s .
$$

Mostremos que a expressão acima não pode ser diretamente aplicada para determinar a força que age sobre um vórtice de intensidade $\Gamma_{k}$, massa $m$ e localizado no ponto $\mathfrak{p}_{k}=\left(x_{k}, y_{k}\right)$. Escolhendo $U$ como sendo o disco de raio $r$ e centrado em $\mathfrak{p}_{k}$ e considerando que a trajetória $\left(x_{k}(t), y_{k}(t)\right), k=1, \cdots, n$, de cada vórtice é uma curva regular, decompondo $\mathbf{v}=\mathbf{v}_{S}+\mathbf{v}_{R}$ onde a componente singular $\mathbf{v}_{s}$ é

$$
\mathbf{v}_{S}:=\frac{\mathbf{i} \Gamma_{k}}{2 \pi} \frac{\mathbf{z}-\mathbf{z}_{k}}{\left|z-z_{k}\right|^{2}}
$$

temos que, a princípio, o termo $-\iint_{U \backslash B} \partial_{t} \mathbf{v} d \mu$ pode ser calculado de três formas diferentes: 
i) Diretamente: $\iint_{U \backslash \mathfrak{p}_{k}} \partial_{t} \mathbf{v}_{S} d \mu=\left.\Gamma_{k}\left(-\dot{y}_{k}, \dot{x}_{k}\right) \ln s\right|_{0} ^{r}+o(r)$.

ii) Considerando que $\mathbf{v}_{S}=\nabla \phi_{S}$ tem-se

$$
\iint_{U \backslash \mathfrak{p}_{k}} \partial_{t} \mathbf{v} d \mu \underset{T e o . D i v .}{\stackrel{?}{=}} \int_{\partial U} \dot{\phi}_{s} \hat{n} d s+o(r)=\frac{\Gamma_{k}}{2}\left(-\dot{y}_{k}, \dot{x}_{k}\right)+o(r) .
$$

iii) Considerando que $\mathbf{v}_{S}=-J \nabla \psi_{S}$ tem-se

$$
\iint_{U \backslash \mathfrak{p}_{k}} \partial_{t} \mathbf{v} d \mu \underset{T e o . D i v .}{\stackrel{?}{=}}-\int_{\partial U} \dot{\psi}_{s} \hat{t} d s+o(r)=\frac{\Gamma_{k}}{2}\left(-\dot{x}_{k}, \dot{y}_{k}\right)+o(r) .
$$

Note que enquanto o primeiro caso não fornece nenhuma informação, os dois últimos são incompatíveis entre si, o que já era esperado pois, em virtude da singularidade do campo de velocidades, o teorema do divergente é aplicado de maneira equivocada. Na próxima seção descrevemos uma maneira alternativa de definir a força que um fluido exerce sobre um vórtice.

\subsection{Vórtices em domínios do plano}

Seja $D$ um domínio regular do plano, no qual considera-se, como hipótese, que têm-se uma distribuição inicial de vorticidade representada pela medida

$$
\omega=\sum_{k=1}^{N} \Gamma_{k} \delta_{\mathfrak{p}_{k}}+\omega_{c}
$$

onde $\omega_{c}$ é constante e $\delta_{\mathfrak{p}_{k}}$ é a medida de Dirac centrada no ponto $\mathfrak{p}_{k}$. Denotamos por $\mathbf{v}$ o campo de velocidades associado a $\omega$ e por $\psi$ a função de corrente correspondente, cuja determinação será descrita no próximo Capítulo. Dada uma sequência $\omega_{l}$ de vorticidades iniciais regulares satisfazendo $\omega_{l} \rightarrow \omega$, existe $T>0$ tal que para todo $t \in[0, T]$ temos

$$
\omega_{l}(t) \rightarrow \omega(t)=\sum_{k=1}^{N} \Gamma_{k} \delta_{\mathfrak{p}_{k}(t)}+\omega_{c}
$$

onde $\mathfrak{p}_{k}(t)$ são curvas regulares e $\omega_{l}(t)$ é a evolução temporal da vorticidade inicial $\omega_{l}$ segundo a equação de Euler. Conforme será apresentado no Capítulo 4, a equação 3.2 é uma consequência imediata da propriedade de localização demonstrada por Marchioro \& Pulvirenti[MP93], cuja formulação mais geral garante que dada uma vorticidade inicial $\omega$ tal que o suporte de $\omega-\omega_{c}$ esteja contido em pequenos discos de raio $\epsilon$, num tempo futuro a evolução $\omega(t)$ de $\omega$ pela equação de Euler é tal que a vorticidade $\omega(t)-\omega_{c}$ permanecerá concentrada em pequenas regiões, isto é, o suporte desta vorticidade estará contido em discos de raio $d_{\epsilon}$ e $\lim _{\epsilon \rightarrow 0} d_{\epsilon} \rightarrow 0$. Este foi o primeiro resultado que fornece uma justificativa rigorosa para as equações da dinâmica de vórtices. Considerando este fato, encontraremos um sistema de equações diferenciais para as curvas $\mathfrak{p}_{k}(t)$ que definem as trajetórias dos vórtices $\Gamma_{k} \delta_{\mathfrak{p}_{k}(t)}$.

Inicialmente, faz-se necessário determinar a força que o fluido exerce sobre um vórtice. Em virtude da impossibilidade de determinar diretamente esta força, definimo-la pelo limite:

$$
\mathcal{F}\left(\mathfrak{p}_{k}\right)=\lim _{l \rightarrow \infty} \mathcal{F}_{l}\left(\mathfrak{p}_{k}\right)
$$

onde $\mathcal{F}_{l}\left(\mathfrak{p}_{k}\right)$ é a força que o fluido correspondente ao campo de velocidades $\mathbf{v}_{l}$ exerce sobre a partícula localizada em $\mathfrak{p}_{k}$ e de massa $m_{k}$ onde a vorticidade concentra-se. Tal como apresentado no início 
deste Capítulo, temos

$$
\mathcal{F}_{l}\left(\mathfrak{p}_{k}\right)=-\iint_{U \backslash\left\{\mathfrak{p}_{k}\right\}} \partial_{t} \mathbf{v}_{l} d \mu-\int_{\partial U} \mathbf{v}_{l}\left(\mathbf{v}_{l}, \hat{n}\right) d s-\int_{\partial U} p_{l} \hat{n} d s,
$$

onde $U$ é uma vizinhança aberta de $\mathfrak{p}_{k}$. Como $\mathbf{v}_{l}=-J \nabla \psi_{l}$, a equação acima escreve-se

$$
\mathcal{F}_{l}\left(\mathfrak{p}_{k}\right)=\iint_{U \backslash\left\{\mathfrak{p}_{k}\right\}} J \nabla \dot{\psi}_{l} d \mu-\int_{\partial U} \mathbf{v}_{l}\left(\mathbf{v}_{l}, \hat{n}\right) d s-\int_{\partial U} p_{l} \hat{n} d s=\int_{\partial U} \dot{\psi}_{l} J \hat{n} d s-\int_{\partial U} \mathbf{v}_{l}\left(\mathbf{v}_{l}, \hat{n}\right) d s-\int_{\partial U} p_{l} \hat{n} d s .
$$

Portanto, para todo $l \in \mathbb{N}$ vale que

$$
\mathcal{F}_{l}\left(\mathfrak{p}_{k}\right)=\int_{\partial U} \dot{\psi}_{l} J \hat{n} d s-\int_{\partial U} \mathbf{v}_{l}\left(\mathbf{v}_{l}, \hat{n}\right) d s-\int_{\partial U} p_{l} \hat{n} d s .
$$

Daí, para determinarmos a força que o $k$-ésimo vórtice de intensidade $\Gamma_{k}$ e massa $m_{k}$ sofre num determinado instante $t \in(-\epsilon, \epsilon)$, consideramos o limite $l \rightarrow \infty$ na equação 3.4 , o que corresponde à concentração de vorticidade $\omega_{l} \rightarrow \sum_{k=1}^{N} \Gamma_{k} \delta_{x_{k}}+\omega_{c}=\omega$, resultando na seguinte expressão para a força sobre o $k$-ésimo vórtice

$$
\mathcal{F}\left(\mathfrak{p}_{k}\right)=\int_{\partial U} \dot{\psi} J \hat{n} d s-\int_{\partial U} \mathbf{v}(\mathbf{v}, \hat{n}) d s-\int_{\partial U} p \hat{n} d s .
$$

Note que na equação acima a função de corrente $\psi$, o campo de velocidades $\mathbf{v}$ e a pressão $p$ são definidos pelo sistema de vórtices com vorticidade de fundo $\omega_{c}$. Além disso, como os integrandos na equação (3.4) são regulares em $\partial U$ e convergem pontualmente para os respectivos integrandos na equação (3.5), o limite considerado está bem definido e portanto a definição 3.3 está bem formulada.

Determinemos, agora, qual deve ser a equação de evolução para os vórtices, isto é, encontremos as equações diferenciais ordinárias que definem as trajetórias $\mathfrak{p}_{k}(t)$ de cada vórtice no intervalo de tempo $(0, \epsilon)$, cuja existência é garantida pela propriedade de localização representada pela equação 3.2. No Capítulo 4 mostra-se que o campo de velocidades correspondente ao escoamento pode ser escrito como a soma de uma componente devida aos vórtices e outra devida à vorticidade de fundo, ou seja,

$$
\mathbf{v}=\mathbf{v}_{v}+\mathbf{v}_{\omega_{c}}
$$

onde $\mathbf{v}_{v}=-J \nabla \psi_{v}$ e $\mathbf{v}_{\omega_{c}}=-J \nabla \psi_{\omega_{c}}$, cujas vorticidades são

$$
\nabla \times \mathbf{v}_{v}=\sum_{k=1}^{n} \Gamma_{k} \text { e } \nabla \times \mathbf{v}_{\omega_{c}}=\omega_{c}
$$

Portanto, considerando que a vorticidade de fundo é constante, temos que

$$
\dot{\psi}=\dot{\psi}_{v} \text { e } \partial_{t} \mathbf{v}=\partial_{t} \mathbf{v}_{v}=-J \nabla \dot{\psi}_{v} .
$$

Além disso, dada uma vizinhança aberta $U$ de $\mathfrak{p}_{k}$, o campo de velocidades devido aos vórtices é irrotacional em $U \backslash\left\{\mathfrak{p}_{k}\right\}$ e portanto existe uma função multivalorada (potencial de velocidade) $\phi_{v}$ tal que em $U \backslash\left\{\mathfrak{p}_{k}\right\}$ vale $\nabla \phi_{v}=\mathbf{v}_{v}$, donde segue-se

$$
\partial_{t} \mathbf{v}_{v}=\nabla \dot{\phi}_{v}
$$

Como apresentado na equação 2.6 da seção 1 , temos que para o campo de velocidades $\mathbf{v}$ vale a 
seguinte identidade

$$
(\mathbf{v} \cdot \nabla) \mathbf{v}=\frac{1}{2} \nabla\left(|\mathbf{v}|^{2}\right)+\omega_{c} \nabla \psi=\nabla\left(\frac{1}{2}|\mathbf{v}|^{2}+\omega_{c} \psi\right) .
$$

Substituindo estes dois últimos resultados na equação de Euler obtemos

$$
\dot{\phi}_{v}+\frac{1}{2}|\mathbf{v}|^{2}+\omega_{c} \psi+p=c t e .
$$

Introduzindo notação complexa, considerando que $\mathbf{v}=\mathbf{v}_{v}+\mathbf{v}_{\omega_{c}}$, definindo o potencial complexo $F=\phi_{v}+i \psi_{v}$ e substituindo os resultados acima na equação 3.5 encontramos que, no aberto $U$, vale a seguinte expressão

$$
\begin{aligned}
\mathcal{F}\left(p_{k}\right) & =\int_{\partial U} \dot{\psi} J \hat{n} d s-\int_{\partial U} \mathbf{v}(\mathbf{v}, \hat{n}) d s-\int_{\partial U} p \hat{n} d s \\
& =\int_{\partial U} \dot{\psi}_{v} i(-i) d z-\int_{\partial U} \mathbf{v} \frac{i}{2}(\mathbf{v} d \bar{z}-\overline{\mathbf{v}} d z)-\int_{\partial U}\left(\dot{\phi}_{v}+\frac{1}{2}|\mathbf{v}|^{2}+\omega_{c} \psi\right) i d z \\
& =-i \int_{\partial U}\left(\dot{\phi}_{v}+i \dot{\psi}_{v}\right) d z-\frac{i}{2} \int_{\partial U} \mathbf{v}^{2} d \bar{z}-\omega_{c} i \int_{\partial U} \psi d z \\
& =-i \int_{\partial U} \dot{F} d z-\frac{i}{2} \int_{\partial U} \mathbf{v}_{v}^{2} d \bar{z}+\left[-\frac{i}{2} \int_{\partial U} 2 \mathbf{v}_{v} \mathbf{v}_{\omega_{c}}+\mathbf{v}_{\omega_{c}}^{2} d \bar{z}-\omega_{c} i \int_{\partial U} \psi d z\right] \\
& =\mathcal{F}_{1}\left(z_{k}\right)+\mathcal{F}_{2}\left(z_{k}\right),
\end{aligned}
$$

onde

$$
\mathcal{F}_{1}\left(z_{k}\right)=-i \int_{\partial U} \dot{F} d z-\frac{i}{2} \int_{\partial U} \mathbf{v}_{v}^{2} d \bar{z} \text { e } \mathcal{F}_{2}\left(z_{k}\right)=-\frac{i}{2} \int_{\partial U} 2 \mathbf{v}_{v} \mathbf{v}_{\omega_{c}}+\mathbf{v}_{\omega_{c}}^{2} d \bar{z}-\omega_{c} i \int_{\partial U} \psi d z \text {. }
$$

Observamos que o cálculo de $\mathcal{F}_{1}\left(z_{k}\right)$ é desenvolvido utilizando apenas teoria de resíduos, enquanto que o cálculo de $\mathcal{F}_{2}\left(z_{k}\right)$ será feito por aproximação.

Façamos o cálculo de $\mathcal{F}_{1}\left(z_{k}\right)$. Considerando que $F(z)=-\frac{i \Gamma_{k}}{2 \pi} \log \left(z-z_{k}\right)+F^{\prime}$, onde $F^{\prime}$ é uma função analítica em $U$, temos

$$
\dot{F}=\frac{i \Gamma_{k} \dot{z}_{k}}{2 \pi} \frac{1}{z-z_{k}}+\dot{F}^{\prime}
$$

onde $\dot{F}^{\prime}$ também é uma função analítica em $U$. Além disso, temos que

$$
\mathbf{v}_{v}=\frac{\mathbf{i} \Gamma_{k}}{2 \pi} \frac{\mathbf{z}-\mathbf{z}_{k}}{\left|z-z_{k}\right|^{2}}+\mathbf{v}_{v}^{\prime}(z, t)
$$

onde $\mathbf{v}_{v}^{\prime}(z, t)$ é tal que seu conjugado complexo resulta numa função analítica em $U$. Portanto, temos

$$
\mathbf{v}^{2}=-\frac{\Gamma_{k}^{2}}{4 \pi^{2}} \frac{\left(z-z_{k}\right)^{2}}{\left|z-z_{k}\right|^{4}}+\mathbf{v}_{v}^{\prime 2}+\frac{i \Gamma_{k} \mathbf{v}_{v}^{\prime}}{\pi} \frac{z-z_{k}}{\left|z-z_{k}\right|^{2}} .
$$

Desta forma, pelo teorema dos resíduos segue-se

$$
\mathcal{F}_{1}\left(z_{k}\right)=-i \frac{i \Gamma_{k} \dot{z}_{k}}{2 \pi} 2 \pi i-\frac{i}{2} \frac{i \Gamma_{k} \mathbf{v}^{\prime}{ }_{v}\left(z_{k}\right)}{\pi}(-2 \pi i)=i \Gamma_{k}\left(\dot{z}_{k}-\mathbf{v}^{\prime}{ }_{v}\left(z_{k}\right)\right),
$$

que é exatamente o resultado de Friedrichs, uma vez que $\mathcal{F}_{1}\left(z_{k}\right)$ é a componente da força correspondente a vorticidade de fundo nula.

Para calcularmos $\mathcal{F}_{2}$, observemos que a força que atua sobre o vórtice em $z_{k}$ independe da vizinhança aberta $U$ considerada, desde que $z_{k}$ seja o único vórtice em $U$. Portanto, desenvolveremos os cálculos tomando como vizinhanças discos de raio $r$ e depois tomaremos o limite $r \rightarrow 0$. Por esta razão, é fácil notar que as componentes regulares em $U$ de $\mathbf{v}_{v}$ e de $\psi$ não darão contribuição alguma no limite, de modo que podemos considerar apenas as partes singulares destes termos, isto 
é, efetivamente temos

$$
\mathbf{v}_{v} \simeq \frac{\mathbf{i} \Gamma_{k}}{2 \pi} \frac{1}{\bar{z}-\bar{z}_{k}} \text { e } \psi \simeq-\frac{\Gamma_{k}}{2 \pi} \ln \left(\left|z-z_{k}\right|\right) .
$$

Para simplificar o cálculo, adotaremos coordenadas polares $(r, \theta)$ e faremos a mudança de coordenadas $z-z_{k} \mapsto w$. Além disso, como já foi dito, consideraremos o aberto $U$ como sendo o disco centrado em $\mathbf{0}$ de raio $r$, de modo que seu bordo é a curva $r(\theta)=r e^{i \theta}, \theta \in(0,2 \pi)$ e portanto temos $d w=i w d \theta$ e $d \bar{w}=-i \bar{w} d \theta$. Com isto, segue-se o desenvolvimento:

$$
\begin{aligned}
\mathcal{F}_{2}\left(z_{k}\right) & =\lim _{r \rightarrow 0}\left[-i \int_{0}^{2 \pi} \mathbf{v}_{\omega_{c}} \frac{\mathrm{i} \Gamma_{k}}{2 \pi} \frac{1}{\bar{w}}(-i \bar{w}) d \theta-\omega_{c} i \int_{0}^{2 \pi}-\frac{\Gamma_{k}}{2 \pi} \ln (r) i r e^{i \theta} d \theta\right] \\
& =-i \Gamma_{k} \mathbf{v}_{\omega_{c}}\left(z_{k}\right)
\end{aligned}
$$

onde observamos, claramente, que a integral envolvendo os termos de ordem superior de $\mathbf{v}_{\omega_{c}}$ tendem a zero quando $r \rightarrow 0$. Com isto, concluímos o resultado desejado:

$$
\mathcal{F}\left(z_{k}\right)=-i \Gamma_{k}\left(\dot{z}_{k}-\left(\mathbf{v}^{\prime}{ }_{v}+\mathbf{v}_{\omega_{c}}\right)\right)=-i \Gamma_{k}\left(\dot{z}_{k}-\mathbf{v}_{R}\right),
$$

onde

$$
\mathbf{v}_{R}=\mathbf{v}^{\prime}{ }_{v}+\mathbf{v}_{\omega_{c}}=\mathbf{v}-\frac{i \Gamma_{k}}{2 \pi} \frac{z-z_{k}}{\left|z-z_{k}\right|^{2}}
$$

é a regularização do campo de velocidades $\mathbf{v}$ obtida removendo a contribuição do $k$-ésimo vórtice.

Para vórtice livre, isto é, se $m_{k}=0$, temos

$$
\dot{z}_{k}=\mathbf{v}_{R}\left(z_{k}\right)
$$

Como será apresentado na próxima seção, esta abordagem torna possível a dedução das equações de movimento para um sistema de vórtices em superfícies.

\subsection{Vórtices em domínios do plano munidos de uma métrica con- forme}

Trataremos, nesta seção, uma situação um pouco mais geral, onde consideramos no domínio $D \subset \mathbb{R}^{2}$ uma variação conforme $g=\lambda^{2} g_{0}$ da métrica euclidiana. Note que esta é a situação encontrada ao utilizarmos coordenadas isotérmicas para representarmos uma superfície. Portanto, o estudo desenvolvido nesta seção aplica-se à representação da dinâmica de vórtice em superfícies utilizando-se coordenadas isotérmicas. Todas as considerações feitas com respeito ao domínio $D$ e às condições de contorno e assintóticas aplicam-se ao caso aqui estudado, lembrando que em domínios ilimitados a vorticidade de fundo $\omega_{c}$ é identicamente nula. Considerando que todas as propriedades que caracterizam a função de Green hidrodinâmica, descritas em Flucher \& Gustafsson ([FG97]), são invariantes por variação conforme da métrica, adotaremos a decomposição $\psi=\psi_{v}+\psi_{\omega_{c}}$ tal como feito na seção anterior. A fim de determinarmos a expressão para a força exercida sobre um vórtice, desenvolveremos os cálculos utilizando coordenadas isotérmicas, o que nos permite adotar notação complexa e aplicar as propriedades de funções complexas, lembrando que os operadores envolvidos deverão ser considerados com respeito à métrica $g=\lambda^{2} g_{0}$. No que diz respeito à vorticidade de um campo de velocidades $\mathbf{v}$, convém apresentar sua definição mais geral para campos em uma superfície riemanniana $(\mathcal{S}, g)$ com forma de volume $\mu$. Conforme apresentado em [AK98], a vorticidade de um campo de velocidades $\mathbf{v}$ em uma variedade de dimensão dois é definida como sendo a função $\omega$ que satisfaz

$$
d\left(\iota_{\mathbf{v}} g\right)=\omega \mu,
$$

onde $d$ representa a derivada exterior. No que segue, utilizamos as mesmas notações bem como consideramos a validade da propriedade de localização, de modo que boa parte do desenvolvimento que se segue será análogo ao que foi feito na seção anterior. 
O seguinte Lema permite-nos escrever alguns operadores e elementos geométricos definidos em $\left(D, \lambda^{2} g_{0}\right)$ em termos dos mesmos operadores e elementos na métrica euclidiana $g_{0}$.

Lema 1 Seja $g=\lambda^{2} g_{0}$. Então $o^{1}$

1) Comprimento de arco: $d s=\lambda d s_{0}$.

2) Elemento de volume: $d \mu=\lambda^{2} d \mu_{0}$

3) Gradiente: $\nabla=\frac{1}{\lambda^{2}} \nabla_{0}$

4) Estrutura complexa: $J=J_{0}=\left(\begin{array}{cc}0 & -1 \\ 1 & 0\end{array}\right)$

5) Derivada covariante: $\nabla_{X} Y=\nabla_{0} X+\frac{1}{\lambda}(X \cdot \lambda) Y+\frac{1}{\lambda}(Y \cdot \lambda) X-g_{0}(X, Y) \frac{1}{\lambda} \nabla_{0} \lambda$,

6) Laplaciano: $\Delta_{g}=\frac{1}{\lambda^{2}} \Delta_{0}$,

7) Função de corrente correspondente à distribuição dos vórtices: A função de corrente $\psi_{v}$ correspondente a um sistema de $n$-vórtices em $D$ de intensidades $\Gamma_{k}, k=1, \cdots, n$, e com circulaçôes prescritas em $\partial D=\sum_{j=1}^{m} C_{j}$ dadas por $c_{v}(j)$ é invariante por variaçấo conforme da métrica. Basta observar que as propriedades que definem a funçâo de Green hidrodinâmica em um domínio do plano são invariantes por variação conforme da métrica (vide Flucher $\mathfrak{G}$ Gustafsson [FG97]).

8) Função de corrente correspondente à vorticidade de fundo constante não nula: Esta componente da função de corrente não é um invariante conforme. De fato, a função de corrente correspondente à vorticidade de fundo $\omega_{c}$ em $\left(D, \lambda^{2} g_{0}\right)$ satisfaz $\Delta \tilde{\psi}_{\omega_{c}}=-\omega_{c}$ e portanto, considerando o resultado do item 6 ) devemos resolver o seguinte problema:

$$
\Delta_{0} \tilde{\psi}_{\omega_{c}}=-\omega_{c} \lambda^{2} \text { em }\left(D, g_{0}\right) \text { e } g\left(\nabla \tilde{\psi}_{\omega_{c}}, \hat{n}\right)=0 \text { em } \partial D,
$$

impondo ainda as prescriçôes das circulaçôes $c_{\omega_{c}}(j)$ de $\tilde{\mathbf{v}}_{\omega_{c}}=-J \nabla \tilde{\psi}_{\omega_{c}}$ ao longo de cada componente $C_{j}$ de $\partial D$.

Demonstração 1 Os itens de 1) a 5) são fatos elementares de geometria cujas demonstraçôes seguem diretamente de cálculos em coordenadas isotérmicas. Uma boa referência para auxilio no desenvolvimento destes cálculos é o livro Riemannian Geometry[dC88]. Os itens 7) e 8), conforme mencionado no enunciado, são decorrência direta do trabalho desenvolvido em [FG9"7].

Um ponto chave no desenvolvimento elaborado na seção anterior é reescrever o termo $\nabla_{\mathbf{v}_{l}} \mathbf{v}_{l}$ de duas maneiras diferentes: a primeira como um gradiente, permitindo eliminar o termo de pressão, enquanto que a segunda é como um divergente de um tensor, o que permite aplicar o teorema de Gauss. Encontremos, portanto, tais expressões utilizando cartas isotérmicas.

\footnotetext{
${ }^{1} \mathrm{O}$ subíndice ${ }_{0}$ sempre representa operadores e elementos geométricos tomados com respeito à métrica euclidiana.
} 
Lema 2 Dada uma função suave $\psi$ definida em um aberto de D, considerando uma parametrização isotérmica com fator conforme $\lambda^{2}$ e definindo $\mathbf{v}=-J \nabla \psi$ e $\mathbf{v}_{0}=-J \nabla_{0} \psi$, de modo que no domínio da parametrização tenhamos $\mathbf{v}=\frac{1}{\lambda^{2}} \mathbf{v}_{0}$, vale que

a) $\nabla_{\mathbf{v}} \mathbf{v}=\nabla\left(\frac{1}{2} g(\mathbf{v}, \mathbf{v})-\omega_{c} \psi\right)$,

b) $\nabla_{\mathbf{v}} \mathbf{v}=\left(\operatorname{div}\left(v^{1} \mathbf{v}\right), \operatorname{div}\left(v^{2} \mathbf{v}\right)\right) \mathbf{v}+g(\mathbf{v}, \nabla \ln \lambda)-g(J \mathbf{v}, \nabla \ln \lambda) J \mathbf{v}$.

Demonstração $\mathbf{2}$ a) Utilizando a identidade 5) do Lema 1 para desenvolver a expressâo $\nabla_{\mathbf{v}} \mathbf{v}$, onde $\mathbf{v}=\frac{1}{\lambda^{2}} \mathbf{v}_{0}$, e aplicando as demais relaçôes do referido Lema, obtemos

$$
\begin{aligned}
\nabla_{\mathbf{v}} \mathbf{v} & =\nabla_{0} \frac{\mathbf{v}_{0}}{\lambda^{2}}\left(\lambda^{-2} \mathbf{v}_{0}\right)+\frac{2}{\lambda}(\mathbf{v} \cdot \lambda) \mathbf{v}-g_{0}\left(\frac{1}{\lambda^{2}} \mathbf{v}_{0}, \frac{1}{\lambda^{2}} \mathbf{v}_{0}\right) \frac{1}{\lambda} \nabla_{0} \lambda \\
& =\frac{1}{\lambda^{2}} \nabla_{0_{\mathbf{v}_{0}}}\left(\frac{1}{\lambda^{2}} \mathbf{v}_{0}\right)+\frac{2}{\lambda^{5}}\left(\mathbf{v}_{0} \cdot \lambda\right) \mathbf{v}_{0}-\frac{1}{\lambda^{5}} g_{0}\left(\mathbf{v}_{0}, \mathbf{v}_{0}\right) \nabla_{0} \lambda \\
& =\frac{2}{\lambda^{5}}\left(\mathbf{v}_{0} \cdot \lambda\right) \mathbf{v}_{0}+\frac{1}{\lambda^{4}} \nabla_{0_{\mathbf{v}_{0}}} \mathbf{v}_{0}+\frac{2}{\lambda^{5}}\left(\mathbf{v}_{0} \cdot \lambda\right) \mathbf{v}_{0}-\frac{1}{\lambda^{5}} g_{0}\left(\mathbf{v}_{0}, \mathbf{v}_{0}\right) \nabla_{0} \lambda \\
& =\frac{1}{\lambda^{4}} \nabla_{0_{\mathbf{v}_{0}}} \mathbf{v}_{0}-\frac{1}{\lambda^{4}} g_{0}\left(\mathbf{v}_{0}, \mathbf{v}_{0}\right) \nabla_{0} \ln \lambda
\end{aligned}
$$

Substituindo a expressão $\nabla_{0_{\mathbf{v}_{\mathbf{0}}}} \mathbf{v}_{\mathbf{0}}=\frac{1}{2} \nabla_{0}\left|\mathbf{v}_{\mathbf{0}}\right|^{2}-\Delta_{0} \psi \nabla_{0} \psi$, apresentada na equação 2.6, encontramos

$$
\begin{aligned}
\nabla_{\mathbf{v}} \mathbf{v} & =\frac{1}{\lambda^{2}}\left[\frac{1}{2 \lambda^{2}} \nabla_{0}\left|\mathbf{v}_{\mathbf{0}}\right|^{2}-\frac{1}{\lambda^{2}} \Delta_{0} \psi \nabla_{0} \psi-\frac{1}{\lambda^{2}}\left|\mathbf{v}_{0}\right|^{2} \nabla_{0} \ln \lambda\right] \\
& =\frac{1}{\lambda^{2}}\left[\frac{1}{2} \nabla_{0}\left(\lambda^{-2}\left|\mathbf{v}_{0}\right|^{2}\right)-\frac{1}{\lambda^{2}} \Delta_{0} \psi \nabla_{0} \psi\right] \\
& =\nabla\left(\frac{1}{2} g(\mathbf{v}, \mathbf{v})-\omega_{c} \psi\right) .
\end{aligned}
$$

b) Considerando $\mathbf{v}=v^{i} \partial_{i}$ temos

$$
\nabla_{\mathbf{v}} \mathbf{v}=v^{i} \nabla_{\partial_{i}}\left(v^{j} \partial_{j}\right)=v^{i} \partial_{i} v^{j} \partial_{j}+v^{i} v^{j} \Gamma_{i j}^{k} \partial_{k}
$$

Como div $\mathbf{v}=0$ vale que

$$
\operatorname{div}\left(v^{j} \mathbf{v}\right)=g\left(\nabla v^{j}, \mathbf{v}\right)=v^{i} \nabla_{\partial_{i}} v^{j}=v^{i} \partial_{i} v^{j}
$$

e portanto a equação (3.13) escreve-se

$$
\nabla_{\mathbf{v}} \mathbf{v}=\left(\operatorname{div}\left(v^{1} \mathbf{v}, \operatorname{div}\left(v^{2} \mathbf{v}\right)\right)+v^{i} v^{j} \Gamma_{i j}^{k} \partial_{k},\right.
$$

onde

$v^{i} v^{j} \Gamma_{i j}^{k} \partial_{k}=\left[\left(v^{1}\right)^{2} \partial_{1} \ln \lambda+2 v^{1} v^{2} \partial_{2} \ln \lambda-\partial_{1} \ln \lambda\left(v^{2}\right)^{2}\right] \partial_{1}+\left[\left(v^{1}\right)^{2} \partial_{2} \ln \lambda+2 v^{1} v^{2} \partial_{1} \ln \lambda-\left(v^{2}\right)^{2} \partial_{2} \ln \lambda\right] \partial_{2}$, pois

$\Gamma_{11}^{1}=\partial_{1} \ln \lambda, \quad \Gamma_{12}^{2}=\partial_{1} \ln \lambda, \quad \Gamma_{21}^{1}=\partial_{2} \ln \lambda, \quad \Gamma_{22}^{2}=\partial_{2} \ln \lambda, \quad \Gamma_{22}^{1}=-\partial_{1} \ln \lambda, \quad \Gamma_{11}^{2}=-\partial_{2} \ln \lambda$.

Com uma simples manipulação algébrica mostra-se que

$$
v^{i} v^{j} \Gamma_{i j}^{k} \partial_{k}=g(\mathbf{v}, \nabla \ln \lambda) \mathbf{v}-g(J \mathbf{v}, \nabla \ln \lambda) J \mathbf{v}
$$

e portanto

$$
\nabla_{\mathbf{v}} \mathbf{v}=\left(\operatorname{div}\left(v^{1} \mathbf{v}\right), \operatorname{div}\left(v^{2} \mathbf{v}\right)\right)+g(\mathbf{v}, \nabla \ln \lambda) \mathbf{v}-g(J \mathbf{v}, \nabla \ln \lambda) J \mathbf{v}
$$

2

O seguinte corolário é imediato:

Corolário 1 Para cada $\mathbf{v}_{l}=-J \nabla \psi_{l}$ vale a seguinte identidade

$\iint_{U} \partial_{t} \mathbf{v}_{l}+\nabla_{\mathbf{v}_{l}} \mathbf{v}_{l} d \mu=\oint_{\partial U}-\dot{\psi}_{l} J \hat{n} d s+\oint_{\partial U} g\left(\mathbf{v}_{l}, \hat{n}\right) \mathbf{v}_{l} d s+\iint_{U}[g(\mathbf{v}, \nabla \ln \lambda) \mathbf{v}-g(J \mathbf{v}, \nabla \ln \lambda) J \mathbf{v}] d \mu$.

\footnotetext{
${ }^{2}$ Como curiosidade, vale notar que $\operatorname{div} \mathbf{v}=d i v_{0} \mathbf{v}+2 g(\mathbf{v}, \nabla \ln \lambda)$ e $\nabla \times \mathbf{v}=\nabla_{0} \times \mathbf{v}-2 g(J \mathbf{v}, \nabla \ln \lambda)$, onde $\nabla \times \mathbf{v}$ é a função vorticidade associada ao campo $\mathbf{v}$.
} 
Considerando a identidade acima e a expressão para a força sobre o vórtice apresentada no início deste trabalho, temos que a força que o fluxo do campo $\mathbf{v}_{l}$ exerce sobre o vórtice de massa $m_{k}$ localizada em $\mathfrak{p}_{k}$ é

$$
\begin{aligned}
\mathcal{F}_{l}\left(\mathfrak{p}_{k}\right)= & -\iint_{U \backslash\left\{\mathfrak{p}_{k}\right\}} \partial_{t} \mathbf{v}_{l}+\nabla_{\mathbf{v}_{l}} \mathbf{v}_{l} d \mu-\int_{\partial U} p_{l} \hat{n} d s \\
= & \oint_{\partial U} \dot{\psi}_{l} J \hat{n} d s-\oint_{\partial U} g\left(\mathbf{v}_{l}, \hat{n}\right) \mathbf{v}_{l} d s-\int_{\partial U} p_{l} \hat{n} d s+ \\
& +\iint_{U}\left[g\left(J \mathbf{v}_{l}, \nabla \ln \lambda\right) J \mathbf{v}_{l}-g\left(\mathbf{v}_{l}, \nabla \ln \lambda\right) \mathbf{v}_{l}\right] d \mu .
\end{aligned}
$$

Posto que a expressão acima vale para todo campo $\mathbf{v}_{l}$, tomando o limite $l \rightarrow \infty$ obtemos a expressão para a força que o fluido correspondente à vorticidade $\omega=\sum_{k=1}^{N} \Gamma_{k} \delta_{\mathfrak{p}_{k}}+\omega_{c}$ exerce sobre o $k$-ésimo vórtice

$$
\begin{aligned}
\mathcal{F}\left(\mathfrak{p}_{k}\right)= & \oint_{\partial U} \dot{\psi} J \hat{n} d s-\oint_{\partial U} g(\mathbf{v}, \hat{n}) \mathbf{v} d s-\int p \hat{n} d s+ \\
& +\iint_{U}[g(J \mathbf{v}, \nabla \ln \lambda) J \mathbf{v}-g(\mathbf{v}, \nabla \ln \lambda) \mathbf{v}] d \mu .
\end{aligned}
$$

Note que utilizando a equação (3.12) e desenvolvendo a mesma argumentação feita na seção anterior, temos

$$
\partial_{t} \mathbf{v}=\nabla \dot{\phi}_{v} \text { e } \dot{\psi}=\dot{\psi}_{v}
$$

Portanto, segue-se

$-\oint_{\partial U} p \hat{n} d s=\iint_{U} \partial_{t} \mathbf{v}+\nabla_{\mathbf{v}} \mathbf{v} d \mu=\iint_{U} \nabla\left(\dot{\phi}_{v}+\frac{1}{2} g(\mathbf{v}, \mathbf{v})-\omega_{c} \psi\right) d \mu=\oint_{\partial U}\left(\dot{\phi}_{v}+\frac{1}{2} g(\mathbf{v}, \mathbf{v})-\omega_{c} \psi\right) \hat{n} d s$.

Substituindo este resultado na equação (3.15) encontramos

$$
\begin{aligned}
\mathcal{F}\left(\mathfrak{p}_{k}\right)= & \oint_{\partial U}\left(\dot{\psi}_{v} J \hat{n}+\dot{\phi}_{v} \hat{n}\right) d s+\frac{1}{2} \oint_{\partial U} g(\mathbf{v}, \mathbf{v}) \hat{n} d s+\oint_{\partial U} g(\mathbf{v}, \hat{n}) \mathbf{v} d s-\int_{\partial U} \omega_{c} \psi \hat{n} d s+ \\
& +\iint_{U \backslash\left\{\mathfrak{p}_{k}\right\}}[g(J \mathbf{v}, \nabla \ln \lambda) J \mathbf{v}-g(\mathbf{v}, \nabla \ln \lambda) \mathbf{v}] d \mu .
\end{aligned}
$$

Considerando que a força que o fluido exerce sobre o vórtice não depende da vizinhança $U$ que utilizamos na equação acima, para efeito de cálculos consideraremos $U$ como sendo o disco geodésico de raio $r$ centrado em $\mathfrak{p}_{k}$ e tomaremos o limite $r \rightarrow 0$.

Agora estamos aptos a apresentar o nosso principal resultado desta seção:

Teorema 1 Sejam $\left(D, \lambda^{2} g_{0}\right)$ e $\psi$ a função de corrente correspondente a um sistema de $n$-vórtices em $D$ com vorticdade de fundo constante e igual a $\omega_{c}$. Então, a força que o fluido exerce sobre o $k$-ésimo vórtice é

$$
\mathcal{F}\left(z_{k}\right)=i \Gamma_{k}\left(\dot{z}_{k}-\mathbf{v}_{R}\right)+\frac{\Gamma_{k}^{2}}{4 \pi} \partial_{\bar{z}} \lambda^{-2}\left(z_{k}\right)
$$

Note que, no dominio de parametrização consideramos a seguinte representação da função de corrente e do campo de velocidades $s^{3}$

$$
\psi(z, t)=-\frac{\Gamma_{k}}{4 \pi} \ln \left(\left|z-z_{k}(t)\right|^{2}\right)+\psi_{R}(z, t) \text { e } \mathbf{v}(z, t)=\frac{i \Gamma_{k}}{2 \pi \lambda^{2}} \frac{z-z_{k}(t)}{\left|z-z_{k}(t)\right|^{2}}+\mathbf{v}_{R}(z, t),
$$

onde $\mathbf{v}(z, t)=-J \nabla \psi(z, t)$ e $\mathbf{v}_{R}(z, t)=-J \nabla \psi_{R}(z, t)$. Naturalmente, como utilizamos notação complexa, vale lembrar que $|\cdot|$ denota o módulo de uma função complexa que, por sua vez, corresponde à norma euclidiana do vetor associado a esta função.

Demonstração 3 Seja $U=B_{r}\left(z_{k}\right)$ o disco geodésico centrado em $z_{k}$ e de raio $r$. Definimos as seguintes funçôes

\footnotetext{
${ }^{3}$ Num domínio do plano, a função de corrente associada a um sistema de $n$-vórtices sempre tem a forma aqui apresentada.
} 
- $\operatorname{Int}_{1}(r):=\oint_{\partial B_{r}\left(z_{k}\right)}\left(\dot{\psi}_{v} J \hat{n}+\dot{\phi}_{v} \hat{n}\right) d s$,

- $\operatorname{Int}_{2}(r):=\underset{\partial B_{r}\left(z_{k}\right)}{\oint} g(\mathbf{v}, \hat{n}) \mathbf{v} d s-\underset{\partial B_{r}\left(z_{k}\right)}{\oint}\left(\omega_{c} \psi-\frac{1}{2} g(\mathbf{v}, \mathbf{v})\right) \hat{n} d s$,

- $\operatorname{Int}_{3}(r):=\underset{B_{r}\left(z_{k}\right) \backslash\left\{\mathfrak{p}_{k}\right\}}{ }[g(J \mathbf{v}, \nabla \ln \lambda) J \mathbf{v}-g(\mathbf{v}, \nabla \ln \lambda) \mathbf{v}] d \mu$.

de modo que

$$
\mathcal{F}\left(\mathfrak{p}_{k}\right)=\lim _{r \rightarrow 0}\left(\operatorname{Int}_{1}(r)+\operatorname{Int}_{2}(r)+\operatorname{Int}_{3}(r)\right) .
$$

Antes de prosseguir com a demonstração, convém lembrar alguns resultados e fazer algumas considerações. Tal como feito na seção anterior, a fim de simplificar alguns cálculos introduziremos notação complexa quando conveniente. Desta forma, temos

a) $\psi(z, \bar{z} ; t)=\frac{-\Gamma_{k}}{4 \pi} \ln \left(\left|z-z_{k}\right|^{2}\right)+\psi_{R}(z, \bar{z} ; t)$

b) $\mathbf{v}_{0}(z, \bar{z} ; t)=\frac{i \Gamma_{k}}{2 \pi} \frac{z-z_{k}}{\left|z-z_{k}\right|^{2}}+\mathbf{v}_{0} R(z, \bar{z} ; t)$

c) $\dot{\psi}=\frac{\Gamma_{k}}{4 \pi}\left(\frac{\dot{z}_{k}}{z-z_{k}}+\frac{\dot{\bar{z}}_{k}}{\bar{z}-\bar{z}_{k}}\right)+\dot{\psi}_{R}$

d) $\left|\mathbf{v}_{0}\right|^{2}=\left|\mathbf{v}_{0} R\right|^{2}+\frac{\Gamma_{k}^{2}}{4 \pi^{2}} \frac{1}{\left|z-z_{k}\right|^{2}}+\frac{\Gamma_{k}}{2 \pi i}\left(\frac{\mathbf{v}_{0} R}{z-z_{k}}-\frac{\overline{\mathbf{v}}_{R}}{\bar{z}-\bar{z}_{k}}\right)$

e) $\lambda^{-2}(z, \bar{z})=\lambda^{-2}\left(z_{k}\right)+\partial_{z} \lambda^{-2}\left(z-z_{k}\right)+\partial_{\bar{z}} \lambda^{-2}\left(\bar{z}-\bar{z}_{k}\right)+\tilde{\lambda}$

onde $\psi_{R}, \tilde{\lambda}$ e $\mathbf{v}_{0} R$ são suaves em $z_{k}$ e a função $\tilde{\lambda}$ satisfaz: $\tilde{\lambda}\left(x_{k}, y_{k}\right)=0, \partial_{x} \tilde{\lambda}\left(x_{k}, y_{k}\right)=0$ e $\partial_{y} \tilde{\lambda}\left(x_{k}, y_{k}\right)=$ 0 .

Observe que a componente singular do campo $\mathbf{v}_{0}$ resulta no surgimento das seguintes singularidades nos integrandos de $I n t_{2}(r)$ e $I n t_{3}(r)$ :

$$
\frac{x}{r^{2}}, \frac{y}{r^{2}}, \frac{1}{r^{2}}, \frac{x^{2}}{r^{4}}, \frac{y^{2}}{r^{4}} \text { e } \frac{x y}{r^{4}},
$$

sendo $r^{2}=x^{2}+y^{2}$. Em virtude disto, apenas o jato de primeira ordem dos termos regulares destes integrandos são relevantes quando tomamos o limite de $r$ tendendo a zero ${ }^{4}$. De fato, se $R(x, y)$ é uma função diferenciável na origem satisfazendo $R(0,0)=0, \partial_{x} R(0,0)=0$ e $\partial_{y} R(0,0)=0$, então é imediato verificar que

$$
\begin{gathered}
\lim _{r \rightarrow 0} \int_{\partial B_{r}(\mathbf{0})} \frac{x}{r^{2}} R(x, y) d s_{0}=0, \lim _{r \rightarrow 0} \int_{\partial B_{r}(\mathbf{0})} \frac{y}{r^{2}} R(x, y) d s_{0}=0, \lim _{r \rightarrow 0} \int_{\partial B_{r}(\mathbf{0})} \frac{1}{r^{2}} R(x, y) d s_{0}=0, \\
\lim _{r \rightarrow 0} \int_{\partial B_{r}(\mathbf{0})} \frac{x^{2}}{r^{4}} R(x, y) d s_{0}=0, \lim _{r \rightarrow 0} \int_{\partial B_{r}(\mathbf{0})} \frac{y^{2}}{r^{4}} R(x, y) d s_{0}=0, \lim _{r \rightarrow 0} \int_{\partial B_{r}(\mathbf{0})} \frac{x y}{r^{4}} R(x, y) d s_{0}=0 . \\
\lim _{r \rightarrow 0} \iint_{B_{r}(\mathbf{0})} \frac{x}{r^{2}} R(x, y) d \mu_{0}=0, \lim _{r \rightarrow 0} \iint_{B_{r}(\mathbf{0})} \frac{y}{r^{2}} R(x, y) d \mu_{0}=0, \lim _{r \rightarrow 0} \iint_{B_{r}(\mathbf{0})} \frac{1}{r^{2}} R(x, y) d \mu_{0}=0, \\
\lim _{r \rightarrow 0} \iint_{B_{r}(\mathbf{0})} \frac{x^{2}}{r^{4}} R(x, y) d \mu_{0}=0, \lim _{r \rightarrow 0} \iint_{B_{r}(\mathbf{0})} \frac{y^{2}}{r^{4}} R(x, y) d \mu_{0}=0, \lim _{r \rightarrow 0} \iint_{B_{r}(\mathbf{0})} \frac{x y}{r^{4}} R(x, y) d \mu_{0}=0 .
\end{gathered}
$$

\footnotetext{
${ }^{4}$ Como visto na seção anterior, a integral $I n t_{1}(r)$ é desenvolvida utilizando teoria de resíduos.
} 
Para verificar estes limites basta utilizar coordenadas polares, reescrevendo a função $R(x, y)$ como $r^{2} \tilde{R}(r, \theta)$. Desta forma, podemos considerar apenas o jato de primeira ordem de todas as funções regulares envolvidas. Isto nos permite, sempre que for conveniente, reescrever os termos regulares relevantes em termos de variáves complexas, fazendo a substituição $x=\frac{1}{2}(z+\bar{z})$ e $y=\frac{1}{2 i}(z-$ $\bar{z})$. Além disso, o fato de tomarmos o limite de $r$ tendendo a zero nos permite considerar outra simplificação útil. Embora as integrais devam todas ser feitas em discos geodesicos centrados na posição do vórtice, na prática podemos calcular as integrais em discos euclidianos centrados no vórtice. Para justificarmos isto, consideremos coordenadas polares geodésicas $(u, v)$ com polo na posição do vórtice. Aqui a coordenada $v$ define uma direção e a coordenada $u$ é o comprimento de arco dos raios geodésicos com origem no polo e direção $v$. Com isto, temos que a curva $u=u_{0}=c t e$ aproxima-se do círculo de raio $u_{0}$ quando $u_{0}$ é suficientemente pequeno. Temos também as seguintes expressões para o comprimento de arco e forma de área nestas coordenadas

$$
d s^{2}=d u^{2}+G(u, v) d v^{2}, \quad d \mu=\sqrt{G} d u d v,
$$

onde $G=u^{2}-\frac{1}{3} K_{0} u^{4}+R_{2}(u, v)$ e $\sqrt{G}=u-\frac{1}{6} K_{0} u^{3}+R_{1}(u, u)$, com $R_{2}(u, v)$ de ordem $n>4$ e $R_{1}(u, u)$ de ordem $n>3$ em $u$, sendo $K_{0}$ a curvatura gaussiana no polo (vide Struik - [Str88]). Estas expressões nos mostram que o erro cometido pela simplificação adotada tende a zero quando $u_{0} \rightarrow 0 .^{5}$

Desenvolvendo a expressão para $\operatorname{Int}_{1}(r)$ obtemos

$$
\operatorname{Int}_{1}(r)=\int_{\partial B_{r}\left(z_{k}\right)}\left(\dot{\psi}_{v} J \hat{n}+\dot{\phi}_{v} \hat{n}\right) d s=-i \underset{\partial B_{r}\left(z_{k}\right)}{\oint} \dot{F} d z
$$

onde $F=\phi_{v}+i \psi_{v}$ é o potencial complexo associado aos vórtices. Considerando que $F(z)=$ $-\frac{i \Gamma_{k}}{2 \pi} \log \left(z-z_{k}\right)+F^{\prime}$, onde $F^{\prime}$ é a contribuição dos demais vórtices, temos

$$
\dot{F}=\frac{i \Gamma_{k} \dot{z}_{k}}{2 \pi} \frac{1}{z-z_{k}}+\dot{F}^{\prime}
$$

onde $\dot{F}^{\prime}$ é uma função analítica em $U$. Portanto, temos

$$
\operatorname{Int}_{1}(r)=i \Gamma_{k} \dot{z}_{k}
$$

Para o cálculo de $\operatorname{Int}_{2}(r)$, observemos que, tal como demonstrado na seção anterior, temos

$$
\lim _{r \rightarrow 0} \oint_{B_{r}\left(z_{k}\right)} \omega_{c} \psi \hat{n} d s=0
$$

isto é, para efeito de limite basta considerarmos

$$
\operatorname{Int}_{2}(r) \simeq \oint_{\partial B_{r}\left(z_{k}\right)}\left(\frac{1}{2} g(\mathbf{v}, \mathbf{v})\right) \hat{n}+g(\mathbf{v}, \hat{n}) \mathbf{v} d s .
$$

Desenvolvendo o integrando e utilizando notação complexa obtemos

$$
\begin{aligned}
\operatorname{Int}_{2}(r) & \simeq \underset{\partial B_{r}\left(z_{k}\right)}{\oint} \frac{1}{2} \lambda^{-2} g_{0}\left(\mathbf{v}_{0}, \mathbf{v}_{0}\right) \hat{n}_{0} d s_{0}+\underset{\partial B_{r}\left(z_{k}\right)}{\oint} \lambda^{-2} g_{0}\left(\mathbf{v}_{0}, \hat{n}_{0}\right) \mathbf{v}_{0} d s_{0} \\
& \simeq-\oint_{\partial B_{r}\left(z_{k}\right)} \frac{i}{2} \lambda^{-2}\left|\mathbf{v}_{0}\right|^{2} d z-\frac{i}{2} \oint_{\partial B_{r}\left(z_{k}\right)} \lambda^{-2}\left(\mathbf{v}_{0}^{2} d \bar{z}-\left|\mathbf{v}_{0}\right|^{2} d z\right) \\
& \simeq-\frac{i}{2} \oint_{\partial B_{r}\left(z_{k}\right)} \lambda^{-2} \mathbf{v}_{0}^{2} d \bar{z} .
\end{aligned}
$$

${ }^{5}$ Isto pode ser formalizado em um Lema. 
Considerando que apenas o jato de primeira ordem dos termos regulares que surgem no integrando de $\operatorname{Int}_{1}(r)$ são relevantes, encontramos o seguinte resultado:

$$
\lim _{r \rightarrow 0} \operatorname{Int}_{2}(r)=\frac{\Gamma_{k}^{2}}{4 \pi} \partial_{\bar{z}} \lambda^{-2}-\lambda^{-2} \Gamma_{k} i \mathbf{v}_{\mathbf{0} R}
$$

onde utilizamos as seguintes integrais complexas

$$
I_{1}:=\oint_{B_{r}(0)} \frac{1}{\bar{z}^{2}} d \bar{z}=0, \quad I_{2}:=\oint_{B_{r}(0)} \frac{z}{\bar{z}^{2}} d \bar{z}=0, \quad I_{3}:=\oint_{B_{r}(0)} \frac{z}{\bar{z}} d \bar{z}=2 \pi i, \quad I_{4}:=\oint_{B_{r}(0)} \frac{1}{\bar{z}} d \bar{z}=-2 \pi i .
$$

Para finalizar, verifiquemos que

$$
\lim _{r \rightarrow 0} \operatorname{Int}_{3}(r)=\mathbf{0} .
$$

De fato, dada uma função suave $f$, se denotarmos por $\mathcal{J}_{1}(f)$ o jato de primeira ordem de $f$, temos:

$$
\begin{aligned}
& \tilde{I}_{1}:=\iint_{B_{r}(0)} \frac{1}{r^{2}} \mathcal{J}_{1}(f) d \mu_{0}=2 \pi \ln r f(\mathbf{0}), \quad \tilde{I}_{2}:=\iint_{B_{r}(0)} \frac{x}{r^{2}} \mathcal{J}_{1}(f) d \mu_{0}=0, \\
& \tilde{I}_{3}:=\iint_{B_{r}(0)} \frac{y}{r^{2}} \mathcal{J}_{1}(f) d \mu_{0}=0, \quad \tilde{I}_{4}:=\iint_{B_{r}(0)} \frac{x^{2}}{r^{4}} \mathcal{J}_{1}(f) d \mu_{0}=f(\mathbf{0}) \pi \ln r \\
& \tilde{I}_{5}:=\iint_{B_{r}(0)} \frac{y^{2}}{r^{4}} \mathcal{J}_{1}(f) d \mu_{0}=f(\mathbf{0}) \pi \ln r, \quad \tilde{I}_{6}:=\iint_{B_{r}(0)} \frac{x y}{r^{4}} \mathcal{J}_{1}(f) d \mu_{0}=0 .
\end{aligned}
$$

Considerando estes resultados e utilizando as expressões de $\lambda^{-2}$ e $\mathbf{v}_{0}$ em notação complexa, concluímos que

$$
\operatorname{Int}_{3,1}(r):=\iint_{B_{r}(\mathbf{0})} g(\mathbf{v}, \nabla \ln \lambda) \mathbf{v} d \mu=\frac{\Gamma_{k}^{2}}{4 \pi} \frac{\ln r}{\lambda^{2}\left(z_{k}\right)} \nabla_{0} \ln \lambda\left(z_{k}\right)+f_{2}(r),
$$

$e^{6}$

$$
\operatorname{Int}_{3,2}(r):=\iint_{B_{r}(\mathbf{0})} g(J \mathbf{v}, \nabla \ln \lambda) J \mathbf{v} d \mu=\frac{\Gamma_{k}^{2}}{4 \pi} \frac{\ln r}{\lambda^{2}\left(z_{k}\right)} \nabla_{0} \ln \lambda\left(z_{k}\right)+f_{1}(r)
$$

onde $\lim _{r \rightarrow 0} f_{1}(r)=0$ e $\lim _{r \rightarrow 0} f_{2}(r)=0$. Portanto, segue-se

$$
\lim _{r \rightarrow 0} \operatorname{Int}_{3}(r)=\lim _{r \rightarrow 0}\left(\operatorname{Int}_{3,2}(r)-\operatorname{Int}_{3,1}(r)\right)=-\lim _{r \rightarrow 0}\left(f_{1}(r)-f_{2}(r)\right)=0 .
$$

Daí, obtemos a expressão para a força sobre o vórtice $z_{k}$

$$
\mathcal{F}\left(z_{k}\right)=\lim _{r \rightarrow 0}\left(\operatorname{Int}_{1}(r)+\operatorname{Int}_{2}(r)+\operatorname{Int}_{3}(r)\right)=i \Gamma_{k} \dot{z}_{k}+\frac{\Gamma_{k}^{2}}{4 \pi} \partial_{\bar{z}} \lambda^{-2}-\lambda^{-2} \Gamma_{k} i \mathbf{v}_{\mathbf{0} R},
$$

donde segue que a equação da dinâmica de um vórtice de intensidade $\Gamma_{k}$ e massa $m_{k}$ é

$$
m_{k} \frac{D}{D t} \dot{z}_{k}=i \Gamma_{k} \dot{z}_{k}+\frac{\Gamma_{k}^{2}}{4 \pi} \partial_{\bar{z}} \lambda^{-2}-\lambda^{-2} \Gamma_{k} i \mathbf{v}_{\mathbf{0} R}
$$

pois, pela segunda lei de Newton, $\mathcal{F}\left(z_{k}\right)=m_{k} \frac{D}{D t} \dot{z}_{k}$. Para o caso em que $m_{k}=0$ temos

$$
\dot{z}_{k}=\lambda^{-2} \mathbf{v}_{\mathbf{0} R}+\frac{\Gamma_{k}}{4 \pi} i \partial_{\bar{z}} \lambda^{-2}=\mathbf{v}_{R}+\frac{\Gamma_{k}}{4 \pi} i \partial_{\bar{z}} \lambda^{-2} .
$$

\footnotetext{
${ }^{6}$ Note que a integral $\operatorname{Int}_{3,1}(r)$ pode ser escrita na forma $\iint_{B_{r}(\mathbf{0})} g(\mathbf{v}, \mathbf{u}) \mathbf{v} d \mu \operatorname{com} \mathbf{u}=\nabla \ln \lambda$ enquanto que a integral $\operatorname{Int}_{3,2}(r)$ pode ser escrita como $J \iint_{B_{r}(\mathbf{0})} g(\mathbf{v}, \mathbf{u}) \mathbf{v} d \mu$ fazendo $\mathbf{u}=-J \nabla \ln \lambda$.
} 
ou ainda, considerando que $\nabla_{0} \equiv 2 \partial_{\bar{z}}$ e $J \equiv i$ obtemos

$$
\dot{\mathfrak{p}}_{k}=\mathbf{v}_{R}\left(\mathfrak{p}_{k}\right)-\frac{\Gamma_{k}}{4 \pi} J \nabla \ln \lambda\left(\mathfrak{p}_{k}\right) .
$$

Este resultado está de acordo com aquele obtido no trabalho clássico de Hally ([Hal]). Como a equação 3.19 faz uso de um sistema de coordenadas particular, convém fazer um teste bastante esclarecedor que consiste em verificar que a equação 3.19 está bem definida, ou seja, mostraremos sua covariância com respeito à mudança conforme de coordenadas. Além de elucidar o resultado obtido, este teste nos permite estabelecer uma comparação entre o problema da representação da dinâmica dos vórtices em coordenadas e a conhecida regra de Routh ([New01] pág. 130), a qual descreve como as equações da dinâmica de vórtices com vorticidade de fundo nula mudam via biholomorfismos de domínios do plano.

Sejam $\Phi_{1}: \mathcal{U} \rightarrow D_{1}$ e $\Phi_{2}: \mathcal{U} \rightarrow D_{2}$ cartas isotérmicas definidas em um mesmo aberto $\mathcal{U} \subset \mathcal{S}$ da superfície Riemanniana $(\mathcal{S}, g)$, de modo que a função de transição $f=\Phi_{1} \circ \Phi_{2}^{-1}: D_{2} \rightarrow D_{1}$ é um biholomorfismo. Assim, dadas as expressões $g_{2}=\lambda_{2}^{2}\left(d x^{2}+d y^{2}\right)$ e $g_{1}=\lambda_{1}^{2}\left(d u^{2}+d v^{2}\right)$ da métrica $g$ nas respectivas coordenadas, então $\lambda_{2}^{2}(z)=\lambda_{1}^{2}(f(z))\left|\partial_{z} f\right|^{2}$. Definindo as componentes $\mathbf{v}_{\lambda_{k}^{2}}=\frac{-i \Gamma_{j}}{4 \pi \lambda_{k}^{2}} \partial_{\bar{p}} \ln \lambda_{k}^{2} \operatorname{com} k=1,2$ e $p=w, z$ respectivamente, temos que

$$
\dot{p}_{j}=\mathbf{v}_{k}\left(p_{j}\right):=\mathbf{v}_{k R}\left(p_{j}\right)+\mathbf{v}_{\lambda_{k}^{2}}\left(p_{j}\right),
$$

onde $p_{j}=w_{j}, z_{j}$ denota a posição do $j$-ésimo vórtice representada nos respectivos sistemas de coordenadas, de modo que $w_{j}=f\left(z_{j}\right)$. Analisaremos como as componentes $\mathbf{v}_{\lambda_{k}^{2}}\left(p_{j}\right)$ e $\mathbf{v}_{k R}\left(p_{j}\right)$ transformam-se por mudança de coordenadas. Inicialmente, vê-se que ${ }^{7}$

$$
D f^{-1} \cdot \mathbf{v}_{\lambda_{1}^{2}}=-\frac{i \Gamma_{j}}{4 \pi \lambda_{1}^{2}(f(z))\left|\partial_{z} f\right|^{2}} \partial_{\bar{z}} \ln \left(\lambda_{1}^{2}(f(z))\right),
$$

enquanto que a componente $\mathbf{v}_{\lambda_{2}^{2}}$ pode ser escrita da seguinte forma

$$
\mathbf{v}_{\lambda_{2}^{2}}(z)=-\frac{i \Gamma_{j}}{4 \pi \lambda_{1}^{2}(f(z))\left|\partial_{z} f\right|^{2}} \partial_{\bar{z}} \ln \left(\lambda_{1}^{2}(f(z))\left|\partial_{z} f\right|^{2}\right),
$$

isto é,

$$
\mathbf{v}_{\lambda_{2}^{2}}=-\frac{i \Gamma_{j}}{4 \pi \lambda_{1}^{2}(f(z))\left|\partial_{z} f\right|^{2}} \partial_{\bar{z}} \ln \left(\lambda_{1}^{2}(f(z))\right)-\frac{\Gamma_{j} i}{4 \pi \lambda_{1}^{2}\left|\partial_{z} f\right|^{2}} \overline{\left(\frac{f^{\prime \prime}}{f^{\prime}}\right)}=D f^{-1} \cdot \mathbf{v}_{\lambda_{1}^{2}}-\frac{\Gamma_{j} i}{4 \pi \lambda_{2}^{2}(z)} \overline{\left(\frac{f^{\prime \prime}}{f^{\prime}}\right)} .
$$

Observe que

$$
\frac{1}{4\left(\frac{f^{\prime \prime}}{f^{\prime}}\right)}=\partial_{\bar{z}} \ln \sqrt{\left|\partial_{z} f\right|}
$$

e portanto, definindo a função harmônica $\phi=\ln \left(\sqrt{\left|\partial_{z} f\right|}\right)$, temos

$$
\mathbf{v}_{\lambda_{2}^{2}}=D f^{-1} \cdot \mathbf{v}_{\lambda_{1}^{2}}-\frac{\Gamma_{j} i}{\pi \lambda_{2}^{2}(z)} \partial_{\bar{z}} \phi
$$

Vejamos agora como a componente regular $\mathbf{v}_{R}$ transforma-se pela mudança de coordenadas. Convém lembrar as expressões (equação 3.17) para a função de corrente

$$
\psi(z, \bar{z} ; t)=-\frac{\Gamma_{j}}{4 \pi} \ln \left(\left|z-z_{j}\right|^{2}\right)+\psi_{R}(z, \bar{z} ; t)
$$

\footnotetext{
${ }^{7}$ Note que se $f(z)=w$ é uma transformação holomorfa então $D f \cdot \mathbf{v}$ é equivalente ao produto de números complexos $\left(\partial_{z} f\right) \mathbf{v}$.
} 
e para o campo de velocidade induzido pelo sistema de vórtices

$$
\mathbf{v}(z ; t)=\frac{i \Gamma_{j}}{2 \pi \mu} \frac{z-z_{j}(t)}{\left|z-z_{j}(t)\right|^{2}}+\mathbf{v}_{R}(z ; t)
$$

onde $\mathbf{v}=-J \nabla \psi$ e $\mathbf{v}_{R}=-J \nabla \psi_{R}$. Desta forma, se $\psi_{1}(w)$ e $\psi_{2}(z)$ são representações da função de corrente nestas coordenadas, temos

$$
\psi_{2}(z)=\psi_{1}(f(z))
$$

e portanto

$$
-\frac{\Gamma_{j}}{4 \pi} \ln \left(\left|z-z_{j}\right|^{2}\right)+\psi_{2 R}(z ; t)=-\frac{\Gamma_{j}}{4 \pi} \ln \left(\left|f(z)-f\left(z_{j}\right)\right|^{2}\right)+\psi_{1 R}(w ; t)
$$

onde $w=f(z)$ e, em particular, $f\left(z_{j}\right)=w_{j}$, donde segue-se

$$
\psi_{2 R}(z ; t)=\psi_{1 R}(f(z) ; t)-\frac{\Gamma_{j}}{4 \pi} \ln \left(\frac{\left|f(z)-w_{j}\right|^{2}}{\left|z-z_{j}\right|^{2}}\right) .
$$

Desenvolvendo $f(z)$ em série numa vizinhaça de $z=z_{j}$ temos

$$
f(z)=f\left(z_{j}\right)+\left(z-z_{j}\right) f^{\prime}\left(z_{j}\right)+\frac{1}{2}\left(z-z_{j}\right)^{2} f^{\prime \prime}\left(z_{j}\right)+O\left(z-z_{j}\right)^{3},
$$

o que nos permite escrever

$$
\left|\frac{f(z)-w_{j}}{z-z_{j}}\right|^{2}=\left|\frac{f(z)-f\left(z_{j}\right)}{z-z_{j}}\right|^{2}=\left|f^{\prime}\left(z_{j}\right)+\frac{1}{2}\left(z-z_{j}\right) f^{\prime \prime}\left(z_{j}\right)+O\left(z-z_{j}\right)^{2}\right|^{2} .
$$

Substituindo o resultado acima na expressão de $\psi_{2 R}$ e derivando com respeito a $\bar{z}$ encontramos a seguinte expressão

$$
\partial_{\bar{z}} \psi_{2 R}=\partial_{\bar{w}} \psi_{1 R}(w) \frac{\partial \bar{w}}{\partial \bar{z}}-\frac{\Gamma_{j}}{4 \pi} \frac{\left(f^{\prime}\left(z_{j}\right)+\frac{1}{2}\left(z-z_{j}\right) f^{\prime \prime}\left(z_{j}\right)+O\left(z-z_{j}\right)^{2}\right)\left(\frac{1}{2} \bar{f}^{\prime \prime}+O\left(\bar{z}-\bar{z}_{j}\right)\right)}{\left|f^{\prime}\left(z_{j}\right)+\frac{1}{2}\left(z-z_{j}\right) f^{\prime \prime}\left(z_{j}\right)+O\left(\bar{z}-\bar{z}_{j}\right)\right|^{2}},
$$

de forma que avaliando a equação $3.24 \mathrm{em} z=z_{j}$ obtem-se

$$
\left.\partial_{\bar{z}} \psi_{2 R}\right|_{z=z_{j}}=\left.\left.\partial_{\bar{w}} \psi_{1 R}\right|_{w=w_{j}} \overline{\partial_{z} f}\right|_{z=z_{j}}-\frac{\Gamma_{j}}{8 \pi} \overline{\left(\frac{f^{\prime \prime}}{f^{\prime}}\right)}\left(z_{j}\right)
$$

donde segue o seguinte resultado

$$
\begin{aligned}
-\left.J \nabla \psi_{2 R}\right|_{z=z_{j}} & \equiv-\left.\left.\frac{2 i}{\lambda_{1}^{2}\left|\partial_{z} f\right|^{2}\left(z_{j}\right)} \partial_{\bar{w}} \psi_{1 R}\right|_{w=w_{j}} \overline{\partial_{z} f}\right|_{z=z_{j}}+\frac{\Gamma_{j} i}{4 \pi \lambda_{2}^{2}} \overline{\left(\frac{f^{\prime \prime}}{f^{\prime}}\right)}\left(z_{j}\right) \\
& =-\left.\frac{2 i}{\lambda_{1}^{2}(w) \partial_{z} f\left(z_{j}\right)} \partial_{\bar{w}} \psi_{1 R}\right|_{w=w_{j}}+\frac{\Gamma_{j} i}{4 \pi \lambda_{2}^{2}} \overline{\left(\frac{f^{\prime \prime}}{f^{\prime}}\right)}\left(z_{j}\right) \\
& =-\left.\frac{2 i}{\lambda_{1}^{2}(w)} \partial_{w} f^{-1} \partial_{\bar{w}} \psi_{1 R}\right|_{w=w_{j}}+\frac{\Gamma_{j} i}{4 \pi \lambda_{2}^{2}} \overline{\left(\frac{f^{\prime \prime}}{f^{\prime}}\right)}\left(z_{j}\right)
\end{aligned}
$$

ou seja,

$$
\mathbf{v}_{2 R}\left(z_{j}\right)=\left.D f^{-1} \cdot \mathbf{v}_{1 R}\right|_{w=w_{j}}+\frac{\Gamma_{j} i}{4 \pi \lambda_{2}^{2}} \overline{\left(\frac{f^{\prime \prime}}{f^{\prime}}\right)}\left(z_{j}\right)=\left.D f^{-1} \cdot \mathbf{v}_{1 R}\right|_{w=w_{j}}+\frac{\Gamma_{j} i}{\pi \lambda_{2}^{2}(z)} \partial_{\bar{z}} \phi .
$$

Desta forma, considerando as equações 3.20 e 3.24 obtemos o resultado desejado

$$
\mathbf{v}_{2}\left(z_{j}\right)=D f^{-1} \cdot \mathbf{v}_{1}\left(w_{j}\right) .
$$

Interessante observar que a equação 3.24 é análoga à equação que relaciona as velocidades de vórtices que estejam em correspondência por biholomorfismos do plano, conforme estabelecido pela regra de 
Routh (equação 3.3.2, página 130 de [New01]).

Os fatos acima relacionados nos possibilita desenvolver uma outra maneira para determinar a dinâmica de um vórtice em um domínio do plano com métrica conforme à euclidiana e com vorticidade de fundo possivelmente não nula. De fato, nota-se que o campo de velocidades u que define a dinâmica de um vórtice em tais condições pode ser escrito na forma

$$
\mathbf{u}=\mathbf{u}_{\omega_{c}}+\mathbf{u}_{\lambda^{2}}+\mathbf{u}_{\phi}
$$

onde $\nabla \times \mathbf{u}_{\omega_{c}}=\omega_{c}, \nabla \times \mathbf{u}_{\lambda^{2}}=\frac{\Gamma}{4 \pi} K$, pois em coordenadas isotérmicas a curvatura gaussiana é dada por

$$
K=-\Delta \ln \lambda,
$$

sendo o termo $\mathbf{u}_{\phi}=\frac{\Gamma_{j} i}{\pi \lambda_{2}^{2}(z)} \partial_{\bar{z}} \phi$ determinado pela escolha de uma função harmônica $\phi$ adequada, de modo que as condições de contorno e assintóticas sejam satisfeitas. Conforme mostrado em [FG97], a dinâmica de um vórtice em um domínio com bordo não admite colisão com o mesmo, de modo que o campo u que define a dinâmica de 1 -vórtice de intensidade $\Gamma$ em $\left(D, \lambda^{2} g_{0}\right)$ pela equação $\dot{z}=\mathbf{u}(z)$ deve ser solução da seguinte equação diferencial parcial:

$$
\left\{\begin{array}{l}
\nabla \times \mathbf{u}=\omega_{c}+\frac{\Gamma}{4 \pi} K, \\
\operatorname{div} \mathbf{u}=0, \\
g(\mathbf{u}, \hat{n})=0 \text { em } \partial D, \\
\text { Prescrição das circulações no bordo }
\end{array}\right.
$$

se $D$ é um domínio limitado do plano, ou

$$
\left\{\begin{array}{l}
\nabla \times \mathbf{u}=\frac{\Gamma}{4 \pi} K \\
\operatorname{div} \mathbf{u}=0 \\
\lim _{|z| \rightarrow \infty}\|\mathbf{u}(z)\|=0 \\
\text { Prescrição das circulações no bordo }
\end{array}\right.
$$

se $D$ é ilimitado. Isto é, o problema de determinar o campo que define a dinâmica de um vórtice com vorticidade de fundo $\omega_{c}$ em $\left(D, \lambda^{2} g_{0}\right)$ é equivalente ao problema de determinar um campo de velocidades livre de divergente, que satisfaz as condições de contorno e assintótica adequadas e cuja vorticidade seja $\left(\omega_{c}+\frac{\Gamma}{4 \pi} K\right)$. Isto mostra que a curvatura gaussiana é uma "fonte" de movimento para os vórtices em domínios com curvatura, tal como afirmado em [BK08]. Note que a formulação do problema definido em $(3.26,3.27)$ não faz uso de nenhum sistema de coordenadas. Para resolve-lo num sistema de coordenadas arbitrário necessitamos aplicar a Lei de Biot-Savart no domínio em questão considerando a representação da métrica $g$ no sistema de coordenadas escolhido. No caso de termos uma parametrização isotérmica com fator conforme $\lambda^{2}$, com base nas observações acima notamos que o problema (3.26) reduz-se ao problema de determinar uma função harmônica $\psi_{0} \mathrm{em}$ $D$ tal que a função $H=\psi_{R}+\frac{\Gamma}{4 \pi} \ln \lambda+\psi_{0}$ satisfaça a condição $\partial_{J \hat{n}} H=0 \mathrm{em} \partial D$, isto é, a função $\psi_{0}$ deve ser solução do seguinte problema

$$
\Delta \psi_{0}=0 \text { em } D \text { e } \partial_{J \hat{n}} \psi_{0}(z)=f(z) \forall z \in \partial D,
$$

onde $f(z)=-\partial_{J \hat{n}}\left(\psi_{R}+\frac{\Gamma}{4 \pi} \ln \lambda\right)$ e $\hat{n}$ é o vetor normal, de modo que a solução de (3.26) é dada por $\mathbf{u}=-J \nabla H$. 


\subsection{Dinâmica de vórtices em superfícies fechadas}

Seja $(\mathcal{M}, g)$ uma superfície compacta sem bordo (fechada). Então a função de Green para o Laplaciano é a função $G(p, q)$ definida em $(\mathcal{M} \times \mathcal{M}) \backslash \mathcal{D}$, onde $\mathcal{D}=\{(x, y) \in \mathcal{M} \times \mathcal{M} ; x=y\}$, e que satisfaz as seguintes propriedades ([Aub98]):

0) $G(p, q)$ é $C^{\infty}$ em $(\mathcal{M} \times \mathcal{M}) \backslash \mathcal{D}$,

1)

$$
\Delta_{q} G(p, q)=-\delta_{p}(q)+\frac{1}{\operatorname{Vol}(\mathcal{M})}
$$

onde $\operatorname{Vol}(\mathcal{M})$ é o volume da superfície,

2) $G(p, q)$ é simétrica: $G(p, q)=G(q, p)$,

3) para toda função contínua $\phi$ em $\mathcal{M}$, temos que $\psi(p)=\int_{\mathcal{M}} G(p, q) \phi(q) d \mu(q)$ é $C^{2}$,

4) $\int_{\mathcal{M}} G(p, q) d \mu(p)=0$.

Desta forma, como a função de corrente para um vórtice de intensidade $\Gamma$ localizado em $p \in \mathcal{M}$ é descrita em termos da função de Green pela expressão

$$
\psi(p)=\Gamma G(p, q),
$$

o termo constante em (3.28) é responsável pelo surgimento de uma vorticidade de fundo uniformemente distribuída (vide [BK08]) que interage com o vórtice resultando numa dinâmica não trivial. Cabe investigar a possibilidade de deduzir a dinâmica de um vórtice em $\mathcal{M}$ utilizando as técnicas aqui apresentadas, assumindo, por hipótese, que a vorticidade de fundo não altera-se com a dinâmica bem como a intensidade do vórtice. De fato, podemos utilizar uma parametrização isotérmica de uma vizinhaça aberta do vórtice para desenvolvermos os cálculos necessários a fim de determinar sua dinâmica. Para tanto, é necessário utilizar uma boa representação da função de Green numa vizinhança da diagonal. Em [BK08] a seguinte representação é apresentada:

$$
G(p, q)=-\frac{1}{2 \pi} \ln (d(p, q))+m(p)+O(d(p, q)),
$$

onde $d(p, q)$ é suficientemente pequeno e $m(p)$ é a função suave denominada capacidade de Robin e definida pela regularização da função de Green na diagonal:

$$
m(p):=\lim _{q \rightarrow p}\left(G(q, p)+\frac{\ln d(q, p)}{2 \pi}\right) .
$$

Utilizando a simetria da função de Green, o seguinte Lema expressa $G(p, q)$ em notação complexa numa carta isotérmica:

Lema 3 Seja $U$ um aberto de $\mathbb{C}$, domínio de uma parametrização isotérmica de uma vizinhança aberta de $\mathfrak{p} \in \mathcal{M}$. Para $U$ "suficientemente pequeno", existe uma função $R\left(z, z^{\prime}\right)$ simétrica e suave em $U \times U$ satisfazendo

$$
R(z, z)=0 \text { e } \partial_{z} R\left(z, z^{\prime}\right)_{z^{\prime}=z}=0
$$

e tal que em $U \times U$ vale a seguinte representação de $G$ :

$$
G\left(z, z^{\prime}\right)=-\frac{1}{2 \pi} \ln d_{0}\left(z, z^{\prime}\right)-\frac{1}{4 \pi} \ln \lambda(z)-\frac{1}{4 \pi} \ln \lambda\left(z^{\prime}\right)+\frac{m(z)}{2}+\frac{m\left(z^{\prime}\right)}{2}+R\left(z, z^{\prime}\right) .
$$

Desta forma, a representação em $U$ da função de corrente para um vórtice de intensidade $\Gamma_{k}$ localizado em $z^{\prime}=z_{k}$ é

$$
\psi(z)=-\frac{\Gamma_{k}}{2 \pi} \ln d_{0}\left(z, z_{k}\right)-\frac{\Gamma_{k}}{4 \pi} \ln \lambda(z)-\frac{\Gamma_{k}}{4 \pi} \ln \lambda\left(z_{k}\right)+\Gamma_{k} \frac{m(z)}{2}+\Gamma_{k} \frac{m\left(z_{k}\right)}{2}+\Gamma_{k} R\left(z, z_{k}\right) .
$$


Portanto, considerando a decomposição da função de corrente em suas componentes singular e regular em $U$ temos

$$
\psi(z)=\psi_{S}(z)+\psi_{R}(z)
$$

onde

$\psi_{S}(z)=-\frac{\Gamma_{k}}{2 \pi} \ln d_{0}\left(z, z_{k}\right)$ e $\psi_{R}(z)=-\frac{\Gamma_{k}}{4 \pi} \ln \lambda(z)-\frac{\Gamma_{k}}{4 \pi} \ln \lambda\left(z_{k}\right)+\Gamma_{k} \frac{m(z)}{2}+\Gamma_{k} \frac{m\left(z_{k}\right)}{2}+\Gamma_{k} R\left(z, z_{k}\right)$.

Embora a componente regular $\psi_{R}$ possua uma dependência temporal implícita $\left(z_{k}=z_{k}(t)\right)$, em virtude da regularidade desta dependência o termo $\dot{\psi}_{R}(z)$ não dará contribuição alguma para a expressão da força sobre o vórtice, de modo que, para efeito de cálculos, podemos considerar a seguinte simplificação

$$
\partial_{t} \mathbf{v} \simeq \nabla \dot{\phi}_{S} \text { e } \dot{\psi} \simeq \dot{\psi}_{S}
$$

Desta forma, o resultado da seção anterior pode ser aplicado ao problema aqui posto, bastando substituir na equação (3.17) a seguinte expressão para $\mathbf{v}_{R}$ :

$$
\mathbf{v}_{R}=-2 \lambda^{-2} i \partial_{\bar{z}} \psi_{R} \text { onde } \psi_{R}(z)=-\frac{\Gamma_{k}}{4 \pi} \ln \lambda(z)-\frac{\Gamma_{k}}{4 \pi} \ln \lambda\left(z_{k}\right)+\Gamma_{k} \frac{m(z)}{2}+\Gamma_{k} \frac{m\left(z_{k}\right)}{2}+\Gamma_{k} R\left(z, z_{k}\right) .
$$

Com isto, a força exercida sobre o vórtice $z_{k}$ é

$$
\mathcal{F}\left(z_{k}\right)=i \Gamma_{k} \dot{z}_{k}-\frac{\Gamma_{k}^{2}}{\lambda^{2}} \partial_{\bar{z}} m
$$

É fácil ver que a expressão acima admite uma simples formulação intrínseca. De fato, a força que age sobre o vórtice de massa $m_{k}$ e trajetória $\mathfrak{p}_{k}$ é $\mathcal{F}\left(\mathfrak{p}_{k}\right)=m_{k} \frac{D}{D t} \dot{\mathfrak{p}}_{k}$ e portanto encontramos a seguinte equação

$$
m_{k} \frac{D}{D t} \dot{\mathfrak{p}}_{k}=\Gamma_{k} J \dot{\mathfrak{p}}_{k}-\frac{\Gamma_{k}^{2}}{2} \nabla m
$$

Fazendo $m_{k}=0$ encontramos que a equação para a dinâmica de 1 vórtice livre

$$
\dot{\mathfrak{p}}_{k}=-\frac{\Gamma_{k}}{2} J \nabla m\left(\mathfrak{p}_{k}\right)
$$

Note que a equação (3.29) define a dinâmica de 1-vórtice livre em qualquer domínio ou superfície, bastando obter a função de Robin hidrodinâmica $m$ correspondente ao domínio do problema. Para o caso de um sistema de $n$-vórtices livres em $\mathcal{M}$, é imediato verificar que as equações de movimento são dadas pelo sistema:

$$
\dot{\mathfrak{p}}_{k}=-J \nabla_{\mathfrak{p}_{k}} H_{v s} \text {, onde } H_{v s}:=\frac{\Gamma_{k}}{2} m\left(\mathfrak{p}_{k}\right)+\sum_{j \neq k} \Gamma_{j} G\left(\mathfrak{p}_{j}, \mathfrak{p}_{k}\right) .
$$

Considerando que a função de Green $G$ para o Laplaciano numa superfície compacta deve satisfazer a equação $\Delta_{z} G\left(z_{0}, z\right)=-\delta_{z_{0}}(z)+1 / A$, onde $A$ é a área da superfície, a função de corrente para um sistema de $n$-vórtices em $\mathcal{M}$ é dada por

$$
\psi(z)=\sum_{k=1}^{n} \Gamma_{k} G\left(z_{k}, z\right),
$$

de modo que

$$
\Delta_{z} \psi(z)=-\sum_{k=1}^{n} \Gamma_{k} \delta_{z_{k}}(z)+\frac{\sum_{k=1}^{n} \Gamma_{k}}{A},
$$


isto é, se $\sum_{k=1}^{n} \Gamma_{k}=0$ então $\Delta_{z} \Psi=-\sum_{k=1}^{n} \Gamma_{k} \delta_{z_{k}}(z)$ e portanto, em domínios do plano, o potencial complexo $F=\phi+i \psi$ associado ao sistema de vórtices é uma função tal que $\frac{d}{d z} F$ é meromorfa. Em virtude das várias propriedades das funções meromorfas e dos resultados de teoria de potencial, o problema de um sistema de $n$-vórtices com circulação total nula é consideravelmente mais fácil de ser tratado. Note que, nestes casos, a depender da geometria do domínio, é possível determinar-se o campo de velocidades do sistema sem o conhecimento explícito da função de Green para o Laplaciano, pois considerando que $\overline{\mathbf{v}}(z)=\frac{d}{d z} F(z)$, basta construir uma função meromorfa com o valor correto dos resíduos para determinar-se o campo de velocidades. Em [SA00] esta ideia é utilizada para a construção do campo de velocidades induzido por três vórtices com circulação total nula em um reticulado do plano, caso em que $\frac{d}{d z} F$ é uma função elíptica.

Outra contribuição do trabalho de Boatto \& Koiller é a formulação hamiltoniana da dinâmica de $n$-vórtices em superfícies, a qual reproduzimos abaixo para o caso de vórtices livres.

Seja $\sum_{k=1}^{n} \Gamma_{k} \delta_{z_{k}}+\omega_{c}$ um sistema de $n$ vórtices livres com vorticidade de fundo $\omega_{c}$ definido na superfície $\mathcal{M}$. Dadas a função de Green hidrodinâmica $G(z, w)$, a função de Robin $m(z)$ e a função de corrente da vorticidade de fundo $\psi_{\omega_{c}}$, definimos a função Hamiltoniana

$$
H=\sum_{l<k} \Gamma_{l} \Gamma_{k} G\left(z_{l}, z_{k}\right)+\sum_{k=1}^{n} \Gamma_{k}^{2}\left(\frac{m\left(z_{k}\right)}{2}+\psi_{\omega_{c}}\left(z_{k}\right)\right)
$$

e a forma simplética

$$
\varpi=\sum_{k=1}^{n} \Gamma_{k} d q_{k} \wedge d p_{k},
$$

onde $z_{k}=\left(q_{k}, p_{k}\right)$ são coordenadas locais definidas em abertos de $\mathcal{M}$. Definindo $\mathbf{z}=\left(z_{1}, z_{2}, \cdots, z_{n}\right) \in$ $\mathcal{M} \times \underset{n}{\cdots} \times \mathcal{M}$, temos que as equações de movimento dos vórtices é um sistema hamiltoniano definido pelo campo $X_{H}$ dado pela expressão

$$
\dot{\mathbf{z}}=X_{H}(\mathbf{z}) \text {, onde } \iota_{X_{H}} \varpi=d H \text {. }
$$




\section{Capítulo 4}

\section{Considerações Adicionais Sobre Dinâmica de Vórtices}

Neste capítulo abordaremos dois aspectos importantes do problema da dinâmica de vórtices. O primeiro trata da determinação da função de corrente de um sistema de vórtices, ponto de partida para a determinação das equações que regem sua dinâmica. O segundo ponto a ser tratado é sobre o problema de valor inicial para a equação de Euler e a propriedade de localização, resultado demonstrado por Marchioro e Pulvirenti [MP93] e utilizado no Capítulo anterior na dedução das equações de movimento de um sistema de vórtices. Também propomos a demostração direta desta propriedade para uma sequência de vorticidades iniciais com simetria radial com respeito às posições iniciais dos vórtices e convergindo fracamente para um sistema de vórtices com vorticidade de fundo constante.

\subsection{Determinação da Função de Corrente}

Seja $D$ um domínio regular do plano no qual consideramos um perfil inicial de vorticidade dado por

$$
\omega(\mathfrak{p})=\sum_{k=1}^{N} \Gamma_{k} \delta_{\mathfrak{p}_{k}}+\omega_{c},
$$

onde $\Gamma_{k} \delta_{\mathfrak{p}_{k}}$ representa o vórtice de intensidade $\Gamma_{k}$ posicionado em $\mathfrak{p}_{k}$ e $\omega_{c}$ é a vorticidade de fundo constante. Consideremos, inicialmente, o caso em que $D$ é limitado. Tomemos uma decomposição da função de corrente $\psi$ associada a um sistema de $n$-vórtices com vorticidade de fundo $\omega_{c}$ dada por $\psi=\psi_{v}+\psi_{\omega_{c}}$, onde $\psi_{v}$ é a contribuição dos vórtices cuja construção é descrita abaixo, e $\psi_{\omega_{c}}$ é uma função de classe $C^{2}$ em $D$, localmente constante em $\partial D$ e cujo laplaciano é igual a $-\omega_{c}$. Assim, o campo de velocidades $\mathbf{v}$ tem a decomposição $\mathbf{v}=\mathbf{v}_{v}+\mathbf{v}_{\omega_{c}}$, onde $\mathbf{v}_{v}=-J \nabla \psi_{v}$ e $\mathbf{v}_{\omega_{c}}=-J \nabla \psi_{\omega_{c}}$, cujas vorticidades são

$$
\nabla \times \mathbf{v}_{v}=\sum_{k=1}^{n} \Gamma_{k} \text { e } \nabla \times \mathbf{v}_{\omega_{c}}=\omega_{c}
$$

Para evitar ambiguidades na determinação de $\psi_{v}$ e $\psi_{\omega_{c}}$ devemos fazer algumas considerações sobre a prescrição de $\omega_{c}$ e das circulações do campo ao longo das componentes conexas de $\partial D=\sum_{j=1}^{m} C_{j}$. Definindo as circulações

$$
c_{v}(j):=\oint_{C_{j}} \mathbf{v}_{v} \cdot \hat{t} d s \text { e } c_{\omega_{c}}(j):=\oint_{C_{j}} \mathbf{v}_{\omega_{c}} \cdot \hat{t} d s
$$

temos

$$
c_{j}:=\oint_{C_{j}} \mathbf{v} \cdot \hat{t} d s=c_{v}(j)+c_{\omega_{c}}(j)
$$


e portanto vale o seguinte resultado

$$
\sum_{j=1}^{m} c_{v}(j)+\sum_{j=1}^{m} c_{\omega_{c}}(j)=\sum_{j=1}^{m} c_{j}=\oint_{\partial D} \mathbf{v} \cdot \hat{t} d s=\sum_{k=1}^{n} \Gamma_{k}+\omega_{c} \text { Área }
$$

onde observamos que no caso em que $\omega_{c}=0$ definimos $c_{\omega_{c}}(j)=0 \forall j=1, \cdots, m$, desde que nesta situação o campo de velocidades deve ser completamente determinado pela distribuição dos vórtices. Além disso, como $\nabla \times \mathbf{v}_{\omega_{c}}=\omega_{c}$ temos

$$
\sum_{j=1}^{m} c_{\omega_{c}}(j)=\oint_{\partial D} \mathbf{v}_{\omega_{c}} \cdot \hat{n} d s=\omega_{c} \text { Área e } \sum_{j=1}^{m} c_{v}(j)=\sum_{k=1}^{n} \Gamma_{k} .
$$

Conforme seja a natureza do problema que deseja-se atacar, temos as seguintes possibilidades para prescrever os dados a fim de definir a dinâmica de $n$-vórtices em um domínio limitado com vorticidade de fundo igual a $\omega_{c}$ :

1) Prescreve-se as circulações totais $c_{j}$, as vorticidades $\Gamma_{k}$ e as circulações $c_{v}(j)$ induzidas pelos vórtices e determina-se as circulações $c_{\omega_{c}}(j)=c_{j}-c_{v}(j)$ do campo de vorticidade de fundo $\omega_{c}$.

2) Prescreve-se as circulações totais $c_{j}$, as vorticidades $\Gamma_{k}$ e as circulações $c_{\omega_{c}}(j)$ induzidas pelo campo $\mathbf{v}_{\omega_{c}}$, determinando-se as circulações $c_{v}(j)=c_{j}-c_{\omega_{c}}(j)$.

3) Prescreve-se as circulações $c_{\omega_{c}}(j)$ e $c_{v}(j)$ bem como as vorticidades $\Gamma_{k}$, ficando as circulações totais definidas por $c_{j}=c_{\omega_{c}}(j)+c_{v}(j)$.

Independentemente da situação física em questão, vale notar que o conjunto de dados

$$
\left(\omega, c_{v}(j), c_{\omega_{c}}(j)\right), k=1, \cdots, n \text { e } j=1, \cdots, m \omega=\sum_{k=1}^{N} \Gamma_{k} \delta_{\mathfrak{p}_{k}}+\omega_{c}
$$

satisfazendo as restrições

$$
\sum_{j=1}^{m} c_{\omega_{c}}(j)=\omega_{c} \text { Área e } \sum_{j=1}^{m} c_{v}(j)=\sum_{k=1}^{n} \Gamma_{k},
$$

determina univocamente $\mathbf{v}_{v}$ e $\mathbf{v}_{\omega_{c}}$. Desta forma, para definirmos a evolução da vorticidade inicial $\omega=\sum_{k=1}^{N} \Gamma_{k} \delta_{\mathfrak{p}_{k}}+\omega_{c}$ em um domínio do plano $D$ cujo bordo $\partial D$ possui $m$ componentes conexas $C_{j}$, devemos prescrever o conjunto de dados 4.1 satisfazendo as restrições 4.2. Desta forma definimos o conjunto de condiçôes iniciais e de contorno para a dinâmica de n-vórtices. Vejamos como é feita a construção de $\psi_{v}$ utilizando-se a Função de Green Hidrodinâmica apresentada no trabalho de Flucher \& Gustafsson[FG97], cuja definição é apresentada a seguir:

Definição 1 A função de Green Hidrodinâmica para um vórtice unitário num dominio limitado $D \subset \mathbb{R}^{2}$ com bordo $\partial D=\sum_{j=1}^{m} C_{j}$ de períodos $\gamma_{1}, \cdots, \gamma_{m}$, respectivamente, sujeitos à restrição $\sum_{j=1}^{m} \gamma_{j}=-1$ é definida como a solução $G_{z}$ do problema

$$
\begin{aligned}
-\Delta G_{z} & =\delta_{z} \text { em } \partial D \\
\partial_{J \hat{n}} G_{z} & =0 \text { em } \partial D \\
\int_{C_{j}} \partial_{\hat{n}} G_{z} & =\gamma_{j} \text { para todo } j \\
\int_{\partial D} G_{z} \partial_{\hat{n}} G_{\xi} & =0 \text { para todo } z, \xi \in D .
\end{aligned}
$$


Conforme o referido texto, o procedimento para determinar a função de corrente $\psi_{v}$ de um sistema de $n$-vórtices em $D$ divide-se em dois casos:

- Caso 1: $\sum_{k=1}^{N} \Gamma_{k} \neq 0$

Considerando as prescrições $c_{v}(j)$ das circulações de $\mathbf{v}_{v}$ ao longo de $C_{j} \in \partial D$, definimos cada período pela expressão:

$$
\gamma_{j}:=\frac{c_{v}(j)}{\sum_{k} \Gamma_{k}}
$$

de modo que a função de corrente é dada por

$$
\psi_{v}=\sum_{k} \Gamma_{k} G_{z_{k}}
$$

- Caso 2: $\sum_{k=1}^{N} \Gamma_{k}=0$

Neste caso podemos escolher os períodos $\gamma_{j}$ arbitrariamente, desde que a restrição já mencionada seja satisfeita. Se todas as circulações $c_{v}(j)$ são nulas a função de corrente é definida por $\psi_{v}=\sum_{k} \Gamma_{k} G_{z_{k}}$, tal como no caso anterior. Na situação mais geral em que nem todas as circulações anulam-se, a função de corrente é dada por

$$
\psi_{v}=\sum_{k} \Gamma_{k} G_{z_{k}}+\psi_{0}
$$

onde $\psi_{0}$ é a função de corrente para um fluxo estacionário e com circulação, sendo harmônica em $D$ e constante em cada $C_{j}$, onde estas constantes são determinadas de modo que $\int_{C_{j}} \partial_{\hat{n}} \psi_{0}=$ $c_{j}$. Isto é possível desde que $\sum_{j} c_{j}=0$, como é o caso.

Vale frisar que a função de corrente $\psi_{v}$ para um sistema de $n$-vórtices sempre pode ser escrita na forma

$$
\psi_{v}(z, \bar{z})=\sum_{k=1}^{n}-\frac{\Gamma_{k}}{4 \pi} \ln \left(\left|z-z_{k}\right|^{2}\right)+\tilde{\psi}_{0}(z, \bar{z}),
$$

onde $\tilde{\psi}_{0}$ é uma função harmônica em $D$. No caso em que o domínio é uma região ilimitada do plano, cujo bordo pode ter ou não componentes ilimitadas, a vorticidade de fundo deve ser identicamente nula e portanto temos $\psi=\psi_{v}$. A função de corrente $\psi_{v}$ para um sistema de $n$-vórtices em um tal domínio também é univocamente definida ([Lin41a, Lin41b]), considerando as prescrições pertinentes. No caso de superfícies fechadas ou em superfícies completas mais gerais, a função de Green hidrodinâmica é exatamente a função de Green para o Laplaciano. Em T. Aubin[Aub98], Steiner[Ste05] e P. Li[PL87], o problema da determinação da função de Green em superfícies é analisado em diversos contextos diferentes.

\subsection{Propriedade de Localização}

O problema de valor inicial para a equação de Euler (PVIEE) em um aberto regular $D$ do plano é dado por

$$
\begin{cases}\partial_{t} \mathbf{v}+(\mathbf{v} \cdot \nabla) \mathbf{v}=-\nabla p, & \text { em } D \times I \\ \operatorname{div}(\mathbf{v})=0, & \text { em } D \times I \\ \mathbf{v}(x, t) \cdot \hat{n}=0, & \text { em } \partial D \times I \\ \mathbf{v}(x, 0)=\mathbf{v}_{0} & \text { em } D,\end{cases}
$$


onde $p$ é a pressão escalar, $\hat{n}$ é a normal exterior, $I=(-\epsilon, \epsilon) \operatorname{com} \epsilon>0$ e $\mathbf{v}_{0}$ é o valor inicial do campo de velocidades. Assumindo suficiente regularidade da velocidade inicial $\mathbf{v}_{0}$, garante-se existência e unicidade da solução do problema de valor inicial 4.3, isto é, existe uma função escalar $p(x ; t)$ e um campo de velocidade $\mathbf{v}(x ; t)$ regulares e que satisfazem 4.3 . Associado à solução da equação de Euler tem-se o fluxo $\phi_{t}: D \rightarrow \mathbb{R}^{2}$ que define a trajetória das partículas e que é obtido como solução da equação

$$
\frac{d}{d t} \phi_{t}(x)=\mathbf{v}\left(\phi_{t}(x), t\right), \phi_{0}(x)=x \quad \forall x \in D \text { e } \forall t \in I .
$$

Outra formulação importante da equação de Euler pode ser obtida tomando-se seu rotacional, o que resulta em uma equação de evolução para a vorticidade

$$
\partial_{t} \omega+(\mathbf{v} \cdot \nabla) \omega=0, \omega(x ; 0)=\omega_{0}(x) \text { em } D \times I,
$$

onde $\omega_{0}=\nabla \times \mathbf{v}_{0}$ é a vorticidade inicial. Note que enquanto a equação de evolução da velocidade depende, também, da determinação da evolução da pressão escalar $p(x ; t)$, as equações do fluxo e da vorticidade, tal como apresentadas acima, dependem da solução $\mathbf{v}(x ; t)$ de 4.3. No entanto, como pode ser visto em [MB02] para o caso em que $D=\mathbb{R}^{2}$, a equação de Euler pode ser definida inteiramente em termos apenas da velocidade, do fluxo ou da vorticidade, havendo equivalência entre estas três formulações desde que haja suficiente regularidade dos dados iniciais. A fim de tratar situações mais gerais, define-se a formulação fraca da Equação de Euler pela expressão[MP93]

$$
\frac{d}{d t} \omega[f]=\omega[\mathbf{v} \cdot \nabla f]+\omega\left[\partial_{t} f\right]
$$

onde $f(x ; t)$ é uma função suave e limitada e

$$
\omega[f]:=\int \omega(x ; t) f(x ; t) d \mu_{x} .
$$

Sabe-se que se a vorticidade inicial satisfaz $\omega(x ; 0) \in L_{1} \cap L_{\infty}$, então existe uma única solução para o problema de valor inicial correspondente a 4.5 satisfazendo $\omega(x ; t) \in L_{1} \cap L_{\infty}$. O seguinte teorema é o primeiro resultado matematicamente rigoroso no sentido de estabelecer uma relação entre a dinâmica de vórtices no plano e soluções da equação de Euler:

Teorema 2 (Marchioro/Pulvirenti[MP93]) Considere uma vorticidade inicial da forma

$$
\omega_{\epsilon}(x, 0)=\sum_{i=1}^{n} \omega_{\epsilon ; i}(x, 0),
$$

onde $\omega_{\epsilon ; i}(x, 0)$ é uma função com sinal definido e suportada em uma região $\Lambda_{\epsilon ; i}$ tal que

$$
\Lambda_{\epsilon ; i} \equiv \operatorname{supp} \omega_{\epsilon ; i} \subset B_{\epsilon}\left(z_{i}\right) ; \quad B_{\epsilon}\left(z_{i}\right) \cap B_{\epsilon}\left(z_{j}\right)=\emptyset \text { se } i \neq j
$$

para $\epsilon$ suficientemente pequeno. Denotamos por

$$
\Gamma_{i}=\int \omega_{\epsilon ; i}(x, 0) d x \in \mathbb{R}
$$

a intensidade do vórtice (independente de $\epsilon$ ). Denotemos por $\omega_{\epsilon}(x, t)$ a evolução temporal de $\omega_{\epsilon}(x, 0)$ segundo a equação de Euler. Então, para uma constante $T>0$ fixa arbitrária, temos que para todo $d>0$, existe $\epsilon_{0}(d, T)$ tal que, se $\epsilon \leq \epsilon_{0}(d, T)$ então

$$
\operatorname{supp} \omega_{\epsilon ; i}(x, t) \subset B_{d}\left(z_{i}(t)\right), \text { para todo } t \in[0, T],
$$


onde $z_{i}(t)$ é a solução do sistema de vórtices

$$
\frac{d}{d t} z_{i}(t)=-\nabla_{i}^{\perp} \frac{1}{2 \pi} \sum_{j \neq i} \ln \left|z_{i}(t)-z_{j}(t)\right|,
$$

desde que a solução exista para $t \in[0, T]$. Além disso, $\epsilon \rightarrow 0$ implica $d \rightarrow 0$.

A condição de que $\epsilon \rightarrow 0$ implica $d \rightarrow 0$ significa que no limite $\epsilon \rightarrow 0$ podemos escolher $d$ arbitrariamente pequeno, o que prova a propriedade de localização. Conforme observado em [MP93], a demonstração do teorema 2 pode ser adaptada para um domínio do plano com bordo. No caso mais geral em que um sistema de vórtices interage com uma vorticidade de fundo não nula, também verifica-se a propriedade de localização, conforme mostrado em [MP93]. Neste caso, faz-se necessário definir o sistema onda-vórtice que, em $\mathbb{R}^{2}$, é dado pelo problema de valor inicial

$$
\left\{\begin{array}{l}
\frac{d}{d t} \phi_{t}(x)=\mathbf{v}\left(\phi_{t}(x)\right)+\sum_{i=1}^{N} \Gamma_{i} K\left(\phi_{t}(x)-z_{i}\right), \quad x \neq z_{i}(0), \\
\frac{d}{d t} z_{i}(t)=\mathbf{v}\left(z_{i}(t), t\right)+\sum_{j=1 ; j \neq i}^{N} \Gamma_{j} K\left(z_{i}(t)-z_{j}(t)\right) \\
\mathbf{v}(\cdot, t)=(K * \omega)(\cdot, t), \\
\omega\left(\phi_{t}(x), 0\right)=\omega_{0}(x) \in L_{1} \cap L_{\infty}, \quad \phi_{0}(x)=x, \quad z_{i}(0)=z_{i} .
\end{array}\right.
$$

Considerando a definição do sistema onda-vórtice acima apresentada, o seguinte teorema devido a Marchioro \& Pulvirenti[MP93] demonstra uma versão mais geral da propriedade de localização

Teorema 3 Considere uma vorticidade inicial dada pela medida

$$
\omega_{\epsilon}(x, 0)=\omega_{0}(x)+\sum_{i=1}^{N} \omega_{\epsilon ; i}(x, 0)
$$

onde $\omega_{\epsilon ; i}(x, 0)$ é uma função com sinal definido e cujo suporte está contido em uma região $\Lambda_{\epsilon ; i}$ tal que

$$
\Lambda_{\epsilon ; i} \equiv \operatorname{supp}_{\epsilon ; i} \subset B_{\epsilon}\left(z_{i}\right) ; \quad B_{\epsilon}\left(z_{i}\right) \cap B_{\epsilon}\left(z_{j}\right)=\emptyset \text { se } i \neq j
$$

para $\epsilon$ suficientemente pequeno, onde $B_{r}(z)$ é a bola centrada em z e de raio $r$. Além disso, a distância entre o conjunto $\left\{z_{1}, \cdots, z_{N}\right\}$ e o suporte de $\omega_{0}(x)$ é maior do que uma constante $r_{0}>0$ fixada, sendo que

$$
\int \omega_{\epsilon ; i}(x, 0) d \mu_{x}=\Gamma_{i}
$$

define a intensidade do $i$-ésimo vórtice e também impomos a restrição

$$
\left|\omega_{\epsilon ; i}(x, 0)\right| \leq \operatorname{cte\epsilon }^{-\eta}, \quad \eta<\frac{8}{3} .
$$

Então, fixada a constante positiva $T>0$, para todo $d>0$ com $d<<r_{0}$, existe $\epsilon_{0}(d, T)$ tal que se $\epsilon<\epsilon_{0}(d, T)$ então

$$
\text { supp } \omega_{\epsilon ; i}(x, t) \subset B_{d}\left(z_{i}(t)\right) \text { para todo } t \in[0, T]
$$

e $d \rightarrow 0$ para $\epsilon \rightarrow 0$. Também verifica-se que, para qualquer função contínua e limitada $f(x)$ vale

$$
\lim _{\epsilon \rightarrow 0} \int \omega_{\epsilon}(x, t) f(x) d \mu_{x}=\int \omega\left(\phi_{t}(x), t\right) f(x) d \mu_{x}+\sum_{i=1}^{N} \Gamma_{i} f\left(z_{i}(t)\right),
$$

onde $\omega_{\epsilon}(x, t)$ é a evolução temporal de $\omega_{\epsilon}(x, 0)$ via equação de Euler, sendo $\omega\left(\phi_{t}(x), t\right)$ e $z_{i}(t)$ soluçôes do sistema onda-vórtice 4.6. 
Apesar do teorema acima ser bastante geral, ele não pode ser aplicado ao caso em que a vorticidade inicial é a sobreposição de um sistema de vórtices com uma vorticidade de fundo constante. Para tratar esta situação, consideramos o resultado apresentado em [LM09], no qual demonstra-se a unicidade da solução do sistema onda-vórtice no caso em que a vorticidade inicial é limitada e constante em uma vizinhança das posições iniciais dos vórtices. No caso em que $\omega_{c}=0$, o teorema 2 garante a validade da propriedade de localização que utilizamos no Capítulo anterior a fim de deduzir as equações da dinâmica de vórtices. Observando que para a dedução apresentada das equações da dinâmica de vórtices necessitamos apenas de uma sequência delta preservada pela equação de Euler, isto é, uma sequência de vorticidades iniciais $\omega_{l} \rightarrow \sum_{k=1}^{N} \Gamma_{k} \delta_{\mathfrak{p}_{k}}+\omega_{c}$ tal que a evolução temporal $\omega_{l}(t)$ pela equação de Euler satisfaz $\omega_{l}(t) \rightarrow \omega(t)=\sum_{k=1}^{N} \Gamma_{k} \delta_{\mathfrak{p}_{k}(t)}+\omega_{c} \operatorname{com} t \in[0, T]$ para algum $T>0$, analisaremos a possibilidade de construir uma sequência delta específica de vorticidades iniciais que seja preservada pela equação de Euler. Embora este não seja um problema relevante para a área de equações diferenciais parciais por tratar-se de uma solução muito específica, acreditamos que tal construção seja de interesse no contexto de sistemas dinâmicos e equações diferenciais ordinárias, uma vez que pode desempenhar o papel de um modelo padrão para ilustrar o que entende-se como dinâmica de vórtices. Além deste fato, tal solução específica permite a elaboração de um texto autosuficiente sobre este tema, dispensando a necessidade de abordar aspectos mais gerais normalmente pertinentes aos domínios das equações diferenciais parciais. Note que, no que diz respeito a tal solução, basta que sua existência seja garantida para um tempo $T>0$ arbitrário, pois este fato é suficiente para o propósito ao qual se destina.

\subsubsection{Sobre a construção de uma sequência delta preservada pela equação de Euler}

Inicialmente, determinemos o campo de velocidades correspondente a uma distribuição de vorticidade com simetria radial, isto é, $\omega=\omega(r(x, y))$, onde $r(x, y)=\sqrt{x^{2}+y^{2}}$. Portanto, a função de corrente $\psi$ também é radial, donde segue que o campo de velocidades é dado por

$$
\mathbf{v}=\nabla^{\perp} \psi(r(x, y))=-\frac{\psi^{\prime}(r)}{r}(-\mathbf{y}, \mathbf{x}),
$$

pois $\frac{\partial \psi}{\partial x}=\frac{\psi^{\prime}(r)}{r} x$ e $\frac{\partial \psi}{\partial y}=\frac{\psi^{\prime}(r)}{r} y$. Além disso, verifica-se que

$$
\omega=-\Delta \psi=-\left(\psi^{\prime \prime}+\frac{1}{r} \psi^{\prime}\right)
$$

e portanto

$$
\psi^{\prime}(r)=-\frac{1}{r} \int_{0}^{r} s \omega(s) d s=-\frac{1}{2 \pi r} \int_{B_{r}(0)} \omega d \mu .
$$

Introduzindo notação complexa e definindo a função

$$
\Gamma(z)=\int_{B_{|z|}(0)} \omega d V
$$

temos que o campo de velocidades associado a $\omega$ é dado por

$$
\mathbf{v}(z)=\frac{i \mathbf{z} \Gamma(z)}{2 \pi|z|^{2}}
$$

onde identificamos o campo vetorial com a função complexa correspondente. Escolhendo $\omega=\omega(r)$ analítica real em $r=0$, é fácil verificar que $\lim _{|z| \rightarrow 0}\|\mathbf{v}(z)\|=0$. A função $\Gamma(z)$ tem uma propriedade bastante interessante: dada uma sequência delta $\omega_{k} \rightarrow \Gamma \delta_{\mathbf{0}}(z)$, onde cada função $\omega_{k}$ é suave e radial 
com suporte no disco $B_{r_{k}}(\mathbf{0})$, sendo $r_{i}<r_{j}$ se $i<j$ e $r_{k} \rightarrow 0$, então, dados $i<j$ têm-se

$$
\Gamma_{j}(z)=\int_{B_{|z|}(0)} \omega_{j} d V=\int_{B_{|z|}(0)} \omega_{i} d V=\Gamma_{i}(z), \forall z \in \mathbb{R}^{2} \backslash B_{r_{j}}(\mathbf{0}),
$$

e portanto

$$
\mathbf{v}_{i}(z)=\mathbf{v}_{j}(z) \forall z \in \mathbb{R}^{2} \backslash B_{r_{j}}(\mathbf{0}) .
$$

Isto significa que a concentração de uma vorticidade radial com suporte num disco de raio $r$ não altera o campo de velocidades fora deste disco. Utilizaremos esta propriedade para construir uma sequência delta de vorticidades iniciais concentrando-se em $n$-vórtices, tais que os campos de velocidades correspondentes coincidam fora de discos de raios arbitrariamente pequenos centrados nos vórtices. Para tanto, dados $n$ pontos $z_{1}, \cdots, z_{n}$ em $\mathbb{R}^{2}$, consideramos $n$ sequências delta $\omega_{j, k} \underset{k \rightarrow \infty}{\rightarrow} \Gamma_{j} \delta_{z_{j}}(z), j=1, \cdots, n$ suaves, radiais e com suporte compacto, convergindo fracamente para o vórtice de intensidade $\Gamma_{j}$ localizado em $z_{j}$. Denotando o suporte de $\omega_{j, k}$ por $\Lambda_{j, k}$, exigimos também que para cada $k \in \mathbb{N}$ tenhamos $\Lambda_{j, k+1} \subset \Lambda_{j, k}$ para todo $j=1, \cdots, n$, isto é, os suportes das vorticidades $\omega_{j, k}$ formam uma sequência encaixante de discos centrados na posição dos vórtices, de modo que o limite de seus diâmetros seja nulo, isto é, $\lim _{k \rightarrow \infty} \operatorname{diam}\left(\Lambda_{j, k}\right)=0$. Feitas estas considerações, definimos

$$
\Gamma_{j, k}(z)=\int_{B_{|z|}\left(z_{j}\right)} \omega_{j, k} d V
$$

de modo que o campo de velocidades $\mathbf{v}_{k}$ associado a $\omega_{k}=\sum_{j=1}^{n} \omega_{j, k}$ é dado por

$$
\mathbf{v}_{k}(z)=\sum_{j=1}^{n} \frac{i\left(z-z_{k}\right) \Gamma_{j, k}(z)}{2 \pi\left|z-z_{k}\right|^{2}}, \quad z \in \mathbb{R}^{2} \backslash\left\{z_{1}, \cdots, z_{n}\right\} .
$$

Note que, pela construção da vorticidade $\omega_{k}$, definindo $\Lambda_{k}=\cup_{j=1}^{n} \Lambda_{j, k}$ temos que $\lim _{k \rightarrow \infty} \Lambda_{k}=\bigcup_{j=1}^{n} z_{j}$ e, para cada $k \in \mathbb{N}$ temos

$$
\mathbf{v}_{k}(z)=\mathbf{v}_{k+1}(z) \forall z \in \mathbb{R}^{2} \backslash \Lambda_{k},
$$

isto é, os campos de velocidades $\mathbf{v}_{k}$ coincidem em todo plano a menos de um número finito de discos cuja área total é arbitrariamente pequena. No caso mais geral em que o domínio seja uma região $D$ regular do plano com bordo, dado o PVIEE correspondente a $\omega_{k}$ onde $\Lambda_{k} \subset D$, temos que o campo de velocidades inicial $\mathbf{v}_{k}$ é dado por

$$
\mathbf{v}_{k}(z)=\sum_{j=1}^{n} \frac{i\left(z-z_{k}\right) \Gamma_{j, k}(z)}{2 \pi\left|z-z_{k}\right|^{2}}+\mathbf{v}_{\partial}(z) z \in D \backslash\left\{z_{1}, \cdots, z_{n}\right\},
$$

onde $\mathbf{v}_{\partial}(z)$ é determinado de modo que o campo resultante $\mathbf{v}_{k}(z)$ seja tangente ao bordo e satisfaça as prescrições de circulação em cada uma das componentes conexas de $\partial D$. Se $D$ é um domínio limitado, pode-se também considerar o caso em que $\omega_{k}=\sum_{j=1}^{n} \omega_{j, k}+\omega_{c}$, onde $\omega_{c}$ é uma vorticidade de fundo constante não-nula e $\omega_{j, k}$ são funções regulares satisfazendo $\omega_{k}(z) \rightarrow \sum_{j=1}^{n} \Gamma_{j} \delta_{z_{j}}(z)$. Neste caso obtemos os seguintes campos de velocidades iniciais

$$
\mathbf{v}_{k}(z)=\sum_{j=1}^{n} \frac{i\left(z-z_{j}\right) \Gamma_{j, k}(z)}{2 \pi\left|z-z_{j}\right|^{2}}+\mathbf{v}_{\omega_{c}}+\mathbf{v}_{\partial}(z),
$$

sendo $\mathbf{v}_{\omega_{c}}$ o campo de velocidades correspondente à vorticidade de fundo. Seja $\phi_{k}(\cdot ; t)$ o fluxo associado à solução $\mathbf{v}_{k}(z ; t)$ do PVIEE para $\mathbf{v}_{k}$. Considerando que $\phi_{k}(\cdot ; t)$ preserva volume e que 
a vorticidade é transportada pelo fluxo, queremos mostrar que existe $T>0$ tal que para todo $t \in[0, T]$ temos $\omega_{k}(z ; t)=\sum_{j=1}^{n} \omega_{j, k}(z ; t)+\omega_{c}$ onde $\omega_{j, k}(z ; t) \rightarrow \Gamma_{j} \delta_{z_{j}(t)}(z), \quad z_{j}(t) \in D$. Para tanto devemos verificar:

i) Dado $\delta>0$, existe $T>0$ tal que para todo $t \in[0, T]$ vale $\phi_{k}\left(z_{j} ; t\right) \in B_{\delta}\left(z_{j}\right) \forall k, j$. De fato, verifica-se que ${ }^{1}$

$$
\mathbf{v}_{k}\left(z_{j}\right)=\sum_{i=1, i \neq j}^{n} \frac{i\left(z_{j}-z_{i}\right) \Gamma_{i, k}\left(z_{j}\right)}{2 \pi\left|z_{j}-z_{i}\right|^{2}}+\mathbf{v}_{\omega_{c}}\left(z_{j}\right)+\mathbf{v}_{\partial}\left(z_{j}\right)
$$

independe de $k$ e portanto, definindo $M_{j}=\left\|\mathbf{v}_{k}\left(z_{j}\right)\right\|$ e considerando que $\dot{\phi}_{k}\left(z_{j} ; 0\right)=\mathbf{v}_{k}\left(z_{j}\right)$, existe $\tilde{T}_{j}>0$ tal que $\left\|\dot{\phi}_{k}\left(z_{j} ; t\right)\right\|<2 M_{j}$ para todo $t \in\left[0, \tilde{T}_{j}\right]$; neste ponto carece mostrar que pode-se determinar $\tilde{T}_{j}$ independentemente de $k$. Assim sendo, definindo $T_{j}=\min \left\{\tilde{T}_{j}, \frac{\delta}{2 M}\right\}$, temos que $\phi_{k}\left(z_{j} ; t\right) \in B_{\delta}\left(z_{j}\right) \forall t \in\left[0, T_{j}\right]$. Fazendo $T=\min \left\{T_{1}, \cdots, T_{N}\right\}$ o resultado fica demonstrado.

ii) Mostrar que para cada $t \in[0, T]$ e para cada $j=1, \cdots, n$, a sequência $\phi_{t}\left(z_{j}\right)$ é convergente. Naturalmente, considerando o resultado do ítem $i$ ) têm-se que qualquer ponto de acumulação de tal sequência pertence ao fecho de $B_{\delta}\left(z_{j}\right)$. Precisamos demonstrar que existe um único ponto de acumulação. Tal fato será consequência da unicidade das soluções da equação de Euler com as condições iniciais dadas pelos elementos da sequência delta.

iii) Definindo $\Lambda_{j, k}(t)=\phi_{k}\left(\Lambda_{j, k}, t\right)$, como o volume é preservado pelo fluxo e $\lim _{k \rightarrow \infty} \operatorname{diam}\left(\Lambda_{j, k}\right)=0$, conclui-se que $\lim _{k \rightarrow \infty} \operatorname{diam}\left(\Lambda_{j, k}(t)\right)=0$ para todo $j=1, \cdots, n$ e para todo $t \in[0, T]$. No entanto esta conclusão não é suficiente. Deve-se mostrar que para cada $j=1, \cdots, n$ e para cada $t \in[0, T]$ o limite $\lim _{k \rightarrow \infty} \Lambda_{j, k}(t)$ é um ponto, aqui denotado por $z_{j}(t)$.

Provadas as proposições acima conclui-se que $z_{j}(t):=\lim _{k \rightarrow \infty} \Lambda_{j, k}(t)=\phi_{k}\left(z_{j}, t\right)$ e portanto, como a vorticidade é transportada pelo fluxo, seque-se o resultado desejado:

$$
\omega_{j, k}(z ; t) \rightarrow \Gamma_{j} \delta_{z_{j}(t)}(z), \quad z_{j}(t) \in D .
$$

A conclusão deste estudo define importante projeto de pesquisa futura.

\footnotetext{
${ }^{1}$ Note que este fato, por si só, já é uma boa justificativa para definir a velocidade do $j$-ésimo vórtice como sendo o limite das velocidades induzidas por uma tal sequência delta quando a vorticidade concentra-se. O problema é mostrar que isto está bem definido considerando a evolução das vorticidades iniciais segundo a equação de Euler.
} 


\section{Capítulo 5}

\section{Exemplos}

\subsection{Disco Unitário}

Nesta seção estudamos a influência da vorticidade de fundo na dinâmica de um traçador passivo com movimento induzido por um vórtice no disco unitário. Tal dinâmica representa a trajetória de uma partícula do fluido e portanto seu estudo nos permite identificar regimes de escoamento laminar e caótico, bem como processos de mistura e formação de regiões de isolamento. Embora este seja um modelo de fluido ideal, seu estudo serve como uma ferramenta auxiliar para a compreensão do processo de transporte e mistura de partículas dentro de um tonel cilíndrico onde introduz-se um rotor que gera um vórtice e posteriormente é retirado, permitindo o livre escoamento do fluido. Considera-se que a escala do modelo físico possibilita a redução a um sistema bidimensional e que o escoamento ocorra num regime que seja razoavelmente aproximado por um fluido ideal. Sabe-se que a integrabilidade da dinâmica está associada a um escoamento laminar, onde as trajetórias das partículas são periódicas ou quasi-periódicas e portanto não ha ocorrência de mistura. A não integrabilidade das equações resulta num movimento errático das partículas e no surgimento de caos, o que inicia um processo de mistura mais ou menos eficiente. O artigo de Zannetti e Franzese[ZF93] estuda o movimento das partículas de um fluido induzido por um vórtice em domínios limitados, sendo o problema aqui apresentado uma adaptação do trabalho desenvolvido em [ZF93] ao caso com vorticidade de fundo constante. Considerando que a integrabilidade da dinâmica de um vórtice no disco unitário é devida à simetria de rotação, uma maneira de obtermos não integrabilidade, e portanto iniciar um processo de mistura, é quebrar a simetria de rotação deformando o contorno do recipiente. No referido artigo mostra-se que o resultado obtido é equivalente à dinâmica resultante da perturbação da órbita circular do vórtice no disco unitário. Descrevemos, na próxima seção, como a introdução da vorticidade de fundo constante interfere na dinâmica do traçador passivo observada a partir de um referencial inercial.

\subsubsection{Determinação da função de corrente}

Aplicando a prescrição descrita no capítulo anterior, determinaremos a função de corrente para um vórtice no disco unitário bem como a hamiltoniana correspondente. Seja $\Gamma$ a intensidade do vórtice e $\omega_{c}$ a vorticidade de fundo constante. Representamos a função de corrente por $\psi=\psi_{v}+\psi_{\omega_{c}}$, onde $\psi_{v}$ é a função de corrente correspondente ao vórtice e $\psi_{\omega_{c}}$ é a função de corrente correspondente à vorticidade de fundo. Lembramos que neste caso as restrições para as circulações implicam que

$$
\oint_{\partial D} \mathbf{v}_{v} \cdot d \mathbf{l}=\Gamma \text { e } \oint_{\partial D} \mathbf{v}_{\omega_{c}} \cdot d \mathbf{l}=\iint_{D} \Delta \psi_{\omega_{c}} d \mu=-\omega_{c}(\text { Área do disco }),
$$


onde $\mathbf{v}_{v}=-J \nabla \psi_{v}$ e $\mathbf{v}_{\omega_{c}}=-J \nabla \psi_{\omega_{c}}$. Dado que a função de Green para o problema de Dirichlet no disco unitário é ${ }^{1}$

$$
G_{D}(z, w)=-\frac{1}{2 \pi} \ln \left(\frac{|z-w|}{|z \bar{w}-1|}\right),
$$

temos $\psi_{v}(z)=\Gamma G_{D}\left(z, z_{0}\right)$, onde $z_{0}$ é a posição do vórtice. Considerando que $\psi_{\omega_{c}}=\omega_{c} \psi_{1}$, para determinar a contribuição da vorticidade de fundo basta resolvermos o problema

$$
\Delta \psi_{1}=1 \text { em } D \text { e } \oint_{\partial D}-J \nabla \psi_{1} \cdot d \mathbf{l}=-(\text { Área do disco })=-\pi .
$$

Utilizaremos um procedimento padrão para determinar $\psi_{1}$ : dada a simetria do domínio, busquemos soluções radiais, isto é, $\psi_{1}=\psi_{1}(r)$ onde $r=\sqrt{x^{2}+y^{2}}$. Assim sendo, derivando implicitamente encontramos

$$
\psi_{1 x x}=\frac{\psi_{1}^{\prime}}{r}+\frac{x^{2}\left(r \psi_{1}^{\prime \prime}-\psi_{1}^{\prime}\right)}{r^{3}} \text { e } \psi_{1 y y}=\frac{\psi_{1}^{\prime}}{r}+\frac{y^{2}\left(r \psi_{1}^{\prime \prime}-\psi_{1}^{\prime}\right)}{r^{3}}
$$

e portanto

$$
\nabla \psi_{1}=\frac{r \psi_{1}^{\prime \prime}+\psi_{1}^{\prime}}{r}
$$

isto é

$$
\psi_{1}^{\prime \prime} r+\psi^{\prime}=r \Rightarrow \frac{d}{d r}\left(\psi_{1}^{\prime} r-\frac{r^{2}}{2}\right)=0 \Rightarrow \psi_{1}^{\prime}-\frac{r^{2}}{2}=c_{1} \Rightarrow \psi_{1}^{\prime}=\frac{r}{2}+\frac{c_{1}}{r} \Rightarrow \psi_{1}(r)=\frac{r^{2}}{4}+c_{1} \ln r+c_{2} .
$$

Como a constante $c_{2}$ é irrelevante escolhemos $c_{2}=0$. A constante $c_{1}$ é nula em virtude da restrição imposta sobre a circulação:

$$
-\pi=\oint_{\partial D}-J \nabla \psi_{1} \cdot d \mathbf{d}=-\pi-2 c_{1} \pi
$$

Portanto, temos

$$
\psi_{\omega_{c}}=\frac{\omega_{c}\left(x^{2}+y^{2}\right)}{4} \text { e } \mathbf{v}_{\omega_{c}}=\frac{\omega_{c}}{2}(y,-x)
$$

donde constatamos que o campo de velocidades correspondente à vorticidade de fundo constante $\omega_{c}$ é uma rotação rígida do fluido com velocidade angular igual a $\omega_{c}$.

\subsubsection{Equações de Movimento}

Considerando o resultado da seção anterior, obtemos que a função de corrente para um vórtice de intensidade $\Gamma$ localizado em $z_{0} \in D$ e com vorticidade de fundo igual a $\omega_{c}$ é dada por

$$
\psi(z)=-\frac{\Gamma}{2 \pi} \ln \left(\frac{\left|z-z_{0}\right|}{\left|z \bar{z}_{0}-1\right|}\right)+\frac{\omega_{c}}{2}|z|^{2} .
$$

Conforme apresentado no Capítulo 3, a Hamiltoniana que define a evolução do vórtice é $H\left(z_{0} ; \bar{z}_{0}\right)=$ $\frac{\Gamma}{2} m\left(z_{0} ; \bar{z}_{0}\right)+\psi_{\omega_{c}}\left(z_{0} ; \bar{z}_{0}\right)$, onde a função de Robin $m$ é determinada pela regularização

$$
m\left(z_{0} ; \bar{z}_{0}\right)=\lim _{z \rightarrow z_{0}}\left(G_{D}\left(z ; z_{0}\right)+\frac{1}{2 \pi} \ln \left|z-z_{0}\right|\right)=\frac{1}{2 \pi} \ln \left(1-\left|z_{0}\right|^{2}\right) .
$$

Portanto, temos

$$
H\left(z_{0} ; \bar{z}_{0}\right)=\frac{\Gamma}{4 \pi} \ln \left(1-\left|z_{0}\right|^{2}\right)+\frac{\omega_{c}}{4}\left|z_{0}\right|^{2},
$$

\footnotetext{
${ }^{1}$ Em virtude da simetria do disco, pode-se determinar a função de Green utilizando o método das imagens com respeito à inversão no disco e simetrizando a função obtida.
} 
donde segue-se a equação de movimento

$$
\dot{z}_{0}=-2 i \partial_{\bar{z}_{0}} H\left(z_{0} ; \bar{z}_{0}\right)=\frac{i \Gamma}{2 \pi} \frac{1}{\frac{1}{z_{0}}-\bar{z}_{0}}-\frac{\omega_{c}}{2} i z_{0},
$$

ou ainda

$$
\dot{\bar{z}}_{0}=\frac{i \Gamma}{2 \pi} \frac{1}{z_{0}-\frac{1}{\bar{z}_{0}}}+\frac{\omega_{c}}{2} i \bar{z}_{0}
$$

cuja solução explícita é

$$
z_{0}(t)=\rho_{0} e^{i \Omega t}, \Omega=\frac{\Gamma}{2 \pi\left(1-\rho_{0}^{2}\right)}-\frac{\omega_{c}}{2},
$$

isto é, o vórtice gira ao longo do círculo de raio $\rho_{0}$ com velocidade angular $\Omega$. Fazendo uma re-escala do tempo e definindo o parâmetro $\lambda=\frac{\pi \omega_{c}}{\Gamma}$ as equações de movimento reduzem-se a

$$
\dot{\bar{z}}_{0}=\frac{i}{z_{0}-\frac{1}{\bar{z}_{0}}}+\lambda i \bar{z}_{0}
$$

sendo sua solução representada por

$$
z_{0}(t)=\rho_{0} e^{i \Omega t}, \Omega=\frac{1}{1-\rho_{0}^{2}}-\lambda
$$

As novas hamiltonianas do vórtice e do traçador, considerando a re-escala do tempo, são dadas respectivamente por $\tilde{H}\left(z_{0}\right)=\frac{1}{2} \ln \left(1-\left|z_{0}\right|^{2}\right)+\frac{\lambda}{2}\left|z_{0}\right|^{2}$ e $\tilde{\psi}(z)=-\ln \left(\frac{\left|z-z_{0}\right|}{\left|1-z \bar{z}_{0}\right|}\right)+\lambda|z|^{2}$. Nestas condições, a dinâmica de um vórtice induz um movimento das partículas do fluido regido pela seguinte equação diferencial não-autônoma

$$
\dot{z}=-2 i \partial_{\bar{z}} \tilde{\psi}\left(z ; z_{0}(t)\right)=i\left(\frac{1}{\bar{z}-\bar{z}_{0}}+\frac{z_{0}}{1-\bar{z} z_{0}}\right)-2 \lambda i z
$$

ou também

$$
\dot{\bar{z}}=-i\left(\frac{1}{z-z_{0}}-\frac{1}{z-\frac{1}{\bar{z}_{0}}}\right)+2 \lambda i \bar{z} .
$$

Introduzindo as variáveis $q=\rho^{2}$ e $p=\theta$, onde $\rho=|z|$ e $\theta=\arg (z)$, obtemos que a equação 5.3 é equivalente a $\dot{w}=-J \nabla H^{\prime}$, onde $w=(q, p)$ e

$$
H^{\prime}(q, p)=2 \tilde{\psi}=\ln \left|\frac{\sqrt{q} e^{i p}-1 / \bar{z}_{0}}{\sqrt{q} e^{i p}-z_{0}}\right|+\lambda q
$$

A fim de eliminar a dependência temporal, escrevemos as equações de movimento da partícula no referencial que desloca-se com o vórtice, o que equivale a adotar a mudança de coordenadas resultantes da transformação canônica obtida pela função geratriz $S=(P+\Omega t) q$, o que resulta nas novas coordenadas conjugadas $Q=q$ e $P=p-\Omega t$. Assim fazendo, obtemos a seguinte hamiltoniana autônoma

$$
H^{\prime \prime}(Q, P)=-\frac{1}{2} \ln \left[\frac{\left(\sqrt{Q} \cos P-\rho_{0}\right)^{2}+Q \sin ^{2} P}{\left(\sqrt{Q} \cos P-1 / \rho_{0}\right)^{2}+Q \sin ^{2} P}\right]+\tilde{\Omega} Q
$$

onde $\tilde{\Omega}=\frac{1}{1-\rho_{0}^{2}}$. Como a equação acima não depende do parâmetro $\lambda$, concluímos que o padrão do movimento das partículas do fluido em relação ao vórtice não muda com a variação da vorticidade de fundo. Este resultado já era esperado pois, como vimos, a vorticidade de fundo constante é gerada por uma rotação rígida do fluido em torno do centro do cilindro, de modo que o movimento das partículas do fluido pode ser decomposto como a composição desta rotação rígida com um movimento em torno do vórtice. No entanto, visto de um referencial inercial, as trajetórias das partículas mudam com a variação da vorticidade de fundo, fato que será ilustrado na próxima 
seção, onde também veremos que a distância do vórtice em relação ao centro do cilindro tem um papel relevante no padrão do escoamento.

\subsubsection{Dinâmica do traçador passivo}

Nesta seção apresentamos algumas ilustrações da dinâmica de um traçador passivo e sua dependência com respeito à variação do parâmetro $\lambda$ e da posição $\rho_{0}$ do vórtice. Na figura 5.1 mostramos como a dinâmica depende da variação da posição inicial do vórtice. Neste caso, além das trajetórias do vórtice e de um traçador passivo no sistema de coordenadas inercial, também representamos as curvas de nível da hamiltoniana $H^{\prime \prime}$, que descreve o sistema no referencial do vórtice.

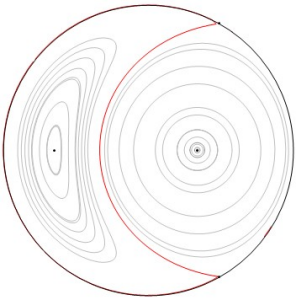

Níveis de $H^{\prime \prime}$ para

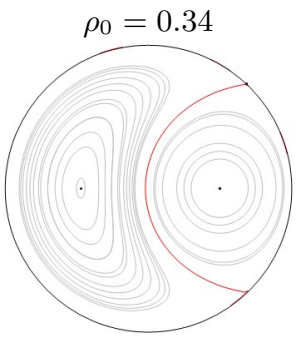

Níveis de $H^{\prime \prime}$ para $\rho_{0}=0.5$

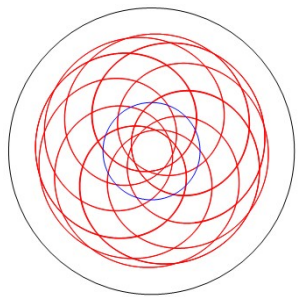

trajetórias do vórtice e do traçador para $\rho_{0}=0.34$

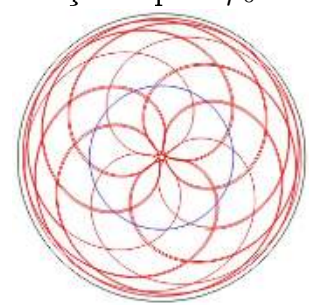

trajetórias do vórtice e do traçador para $\rho_{0}=0.5$

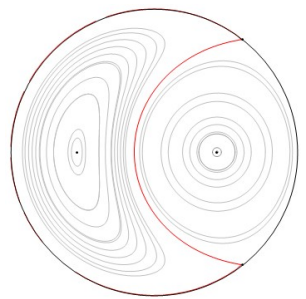

Níveis de $H^{\prime \prime}$ para

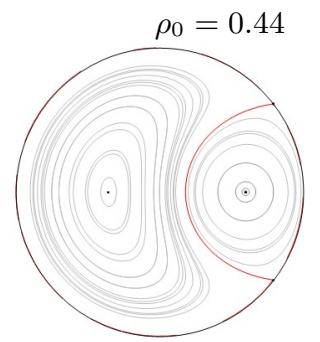

Níveis de $H^{\prime \prime}$ para $\rho_{0}=0.6$

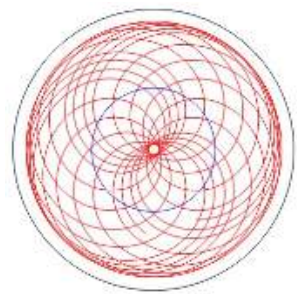

trajetórias do vórtice e do traçador para $\rho_{0}=0.44$

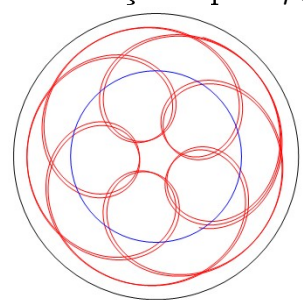

trajetórias do vórtice e do traçador para $\rho_{0}=0.6$

Figura 5.1: As curvas em vermelho (versão eletrônica) nos gráficos dos níveis de $H^{\prime \prime}$ representam as separatrizes heteroclínicas conectando as singularidades hiperbólicas localizados no bordo do disco. Nos gráficos das trajetórias, a do vórtice é representada pelo círculo azul (versão eletrônica) e a do traçador passivo pela curva vermelha (versão eletrônica), sendo a posição inicial do vórtice dada por $\left(\rho_{0}, 0\right)$ enquanto que a do traçador passivo é a mesma em todos os gráficos: $(0.3,-0.5)$.

Para os valores considerados de $\rho_{0}$, não verificamos nenhuma mudança significativa na topologia das curvas de nível. Verificamos sempre a existência de dois pontos elípticos, um na posição do vórtice e outro um ponto elíptico regular localizado no mesmo diâmetro que passa pelo vórtice mas do lado oposto com respeito à origem. Além disso, verifica-se a existência de dois pontos hiperbólicos no bordo que delimita o escoamento, ligados por uma conexão heteroclínica que separa o domínio do fluido em duas regiões: uma é constituída de pontos que rotacionam em torno do vórtice e a outra é formada pelos pontos que rotacionam em torno do segundo ponto elíptico. Em [ZF93] é mostrado que a quebra de simetria resultante de uma perturbação do bordo provoca o surgimento de caos neste sistema, originando o emaranhamento das órbitas heteroclínicas e um processo de mistura das partículas do fluido.

Como já mencionado, a variação da vorticidade de fundo não interfere em nada no movimento de um traçador em relação ao vórtice. Considerando que a dinâmica de um traçador neste domínio é a resultante da combinação de uma rotação rígida com o movimento em relação ao vórtice, embora este último não seja afetado pela vorticidade de fundo o primeiro sofre interferência, de modo que a vorticidade de fundo pode definir, por exemplo, quando a órbita de um traçador passivo será periódica ou quasi-periódica. No caso em que $\omega_{c}$ e $\Gamma$ têm sinais opostos, $\lambda$ será negativo e portanto a velocidade angular do vórtice, dada por $\Omega=\frac{1}{1-\rho_{0}^{2}}-\lambda$, será sempre positiva. Esta situação corresponde ao caso em que a rotação rígida e o movimento induzido pelo vórtice têm sentidos opostos. 


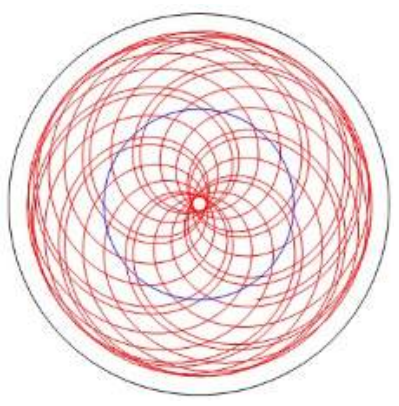

$\lambda=-0.5$

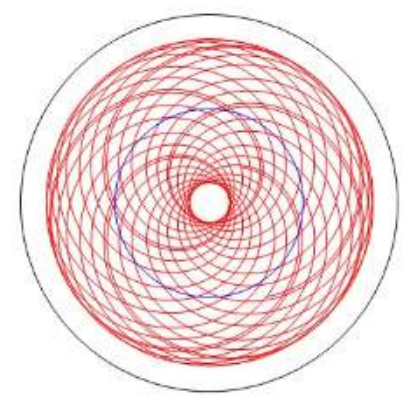

$\lambda=-1$

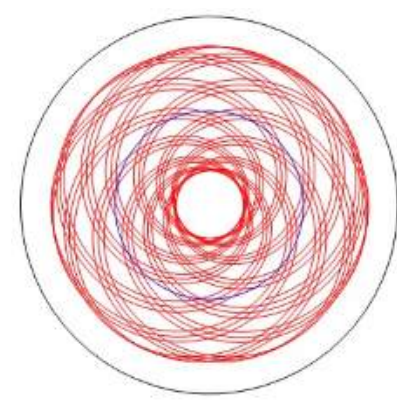

$\lambda=-1.5$

Figura 5.2: Trajetórias do traçador passivo com variação negativa do parâmetro $\lambda$. Posição inicial do vórtice: $(0.5,0)$; posição inicial do traçador passivo: $(0.3,-0.5)$.

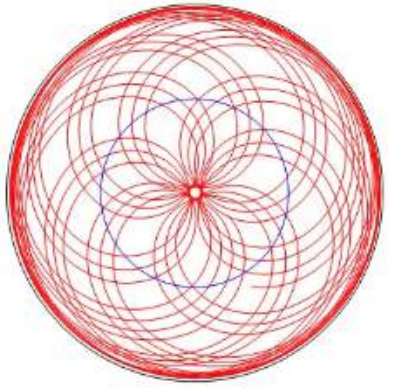

$\lambda=0.1$
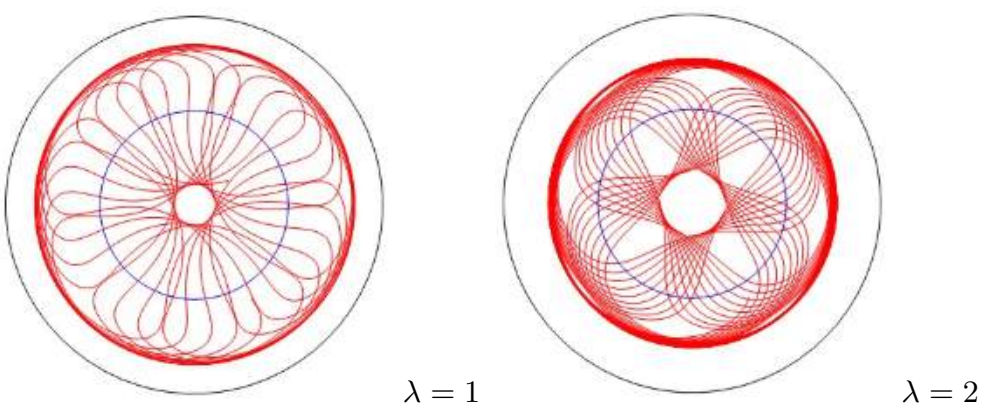

Figura 5.3: Trajetórias do traçador passivo com variação positiva do parâmetro $\lambda$. Posição inicial do vórtice: $(0.5,0)$; posição inicial do traçador passivo: $(0.3,-0.5)$.

No caso em que $\omega_{c}$ e $\Gamma$ têm o mesmo sinal, isto é, $\lambda>0$, as rotações induzidas pelo vórtice e pela vorticidade de fundo terão o mesmo sentido, de modo que para algum valor de $\omega_{c}$ o vórtice fica parado. Esta situação é ilustrada na figura 5.4.

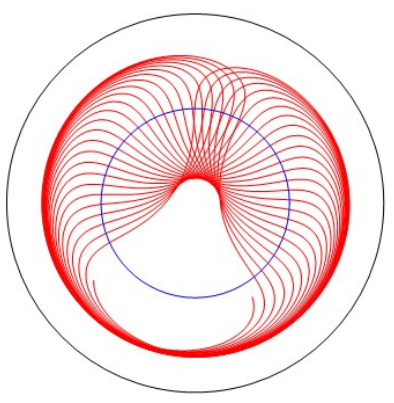

$\lambda=1.3$

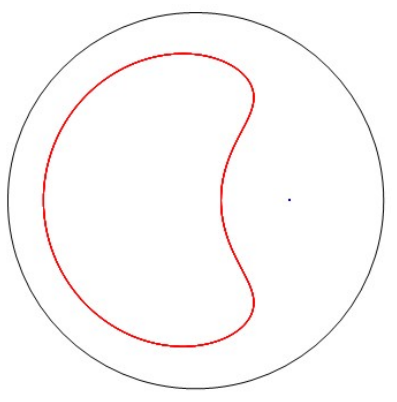

$\lambda=1.33$

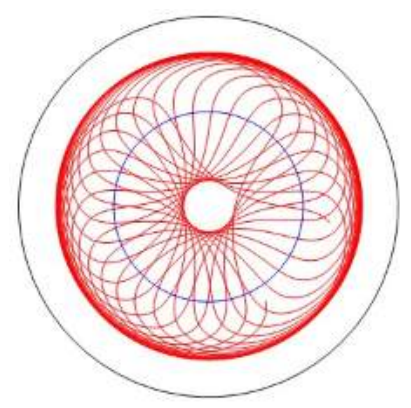

$\lambda=1.4$

Figura 5.4: Trajetórias do traçador passivo em uma vizinhança do valor crítico. Posição inicial do vórtice: $(0.5,0)$; posição inicial do traçador passivo: $(0.3,-0.5)$. 


\subsection{Dois vórtices no toro plano}

O estudo apresentado nesta seção foi fortemente motivado pelo trabalho de Stremler \& Aref[SA00], no qual mostra-se a complexidade e riqueza da dinâmica de três vórtices com vorticidade total nula em um reticulado do plano. Mesmo sendo um sistema integrável, os autores destacam a complexidade das trajetórias dos vórtices e suas bifurcações em diferentes regimes de movimento. Apesar da dinâmica de dois vórtices no toro plano com circulação total nula ser trivial, o caso em que a circulação total é diferente de zero pode ser reduzido ao estudo da evolução da posição relativa entre os vórtices, cuja hamiltoniana $H$ é dada pela função de Green para o laplaciano no toro plano, de modo que o sistema resultante possui dinâmica não-trivial e seu comportamento qualitativo é completamente descrito pela topologia das curvas de nível de $H$. Em [Str10] é descrita a dinâmica de dois vórtices em um reticulado do plano com meio-períodos dados por $w_{1}$ e $w_{2}$, onde $w_{1}$ é um número real e $w_{2}$ assume os seguintes valores: $i w_{1},(1+\sqrt{3} i) w_{1} / 2$ e $(1 / \sqrt{3}+i) w_{1}$. Completamos o estudo deste problema desenvolvendo uma descrição numérica das bifurcações das curvas de nível da função de Green do toro plano considerando variações do parâmetro modular $\tau=w_{2} / w_{1}$ e explicando as estruturas destes diagramas em termos da ação do grupo modular, o qual estabelece correspondência entre toros biholomorficamentes equivalentes.

\subsubsection{Determinação da função de corrente no toro plano}

Dados $g_{1}, g_{2}$ geradores de um reticulado $\Lambda\left(g_{1}, g_{2}\right)=\left\{m g_{1}+n g_{2}\right\}$, onde $m, n \in \mathbb{Z}$ e $g_{2} / g_{1} \in \mathbb{C} \backslash \mathbb{R}$, a ação dos elementos $\lambda=m g_{1}+n g_{2}$ de $\Lambda$ em $\mathbb{C}$ pela translação

$$
\lambda(z)=z+m g_{1}+n g_{2}
$$

define uma estrutura de grupo em $\Lambda$, sendo a projeção $\pi: \mathbb{C} \rightarrow \mathbb{C} / \Lambda$ a aplicação que associa a cada elemento $z$ de $\mathbb{C}$ sua classe de equivalência $[z] \in \mathbb{C} / \Lambda$ definida por

$$
[z]:=\{p \in \mathbb{C} \mid \exists \lambda \in \Lambda ; \lambda(p)=z\} .
$$

Deste modo, o conjunto $\mathbb{C} / \Lambda$ herda as estruturas topológica e complexa de $\mathbb{C}$ definidas naturalmente a partir da projeção, tornando-o, portanto, uma superfície de Riemann de gênero 1, ou seja, um toro. Uma representação geométrica desta superfície no plano é obtida pela identificação dos lados opostos $l_{1}, l_{1}^{\prime}$ e $l_{2}, l_{2}^{\prime}$ do paralelogramo $P$ definido pelos pontos $\mathbf{0}, g_{1}$ e $g_{2}$, conforme ilustrado na figura abaixo.

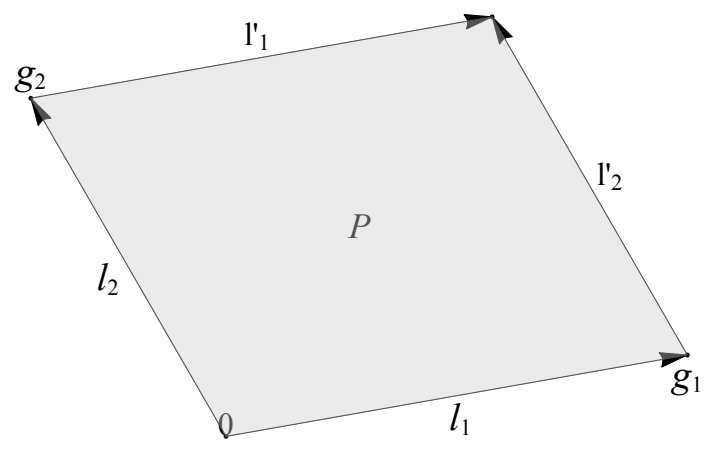

Figura 5.5: O dominio fundamental para um toro plano é um paralelogramo $\mathcal{P}$ cujos lados opostos são identificados preservando a orientação.

O estudo desenvolvido nesta seção considera toros obtidos a partir de reticulados normalizados, isto é, seus geradores são dados por $g_{1}=1$ e $g_{2}=\tau \in \mathbb{C} \backslash \mathbb{R}$. Note que a transformação conforme $f(z)=z / g_{1}$ mapeia um reticulado $\Lambda\left(g_{1}, g_{2}\right)$ no reticulado normalizado $\Lambda(1, \tau)$, onde $\tau=g_{2} / g_{1} \in$ $\mathbb{C} \backslash \mathbb{R}$, ou seja, por meio de uma rotação e uma reescala de comprimento obtemos um reticulado normalizado denotado simplificadamente por $\Lambda(\tau)$, sendo $T(\tau)$ o toro correspondente. 
A função de Green para o Laplaciano no toro plano é a única função simétrica $G(z, w)$, definida em $T \times T$, que é regular fora da diagonal e que satisfaz, no sentido de distribuição, a seguinte equação

$$
\Delta_{z} G(z, w)=-\delta_{w}(z)+\frac{1}{|T|}
$$

e $\int_{T} G(z, w) d \mu_{z}=0$, onde $\delta_{w}$ é a medida de Dirac com singularidade em $z=w$ e $|T|=b$ é a área de $T(\tau)$ com parâmetro modular $\tau=a+b i, b>0$. Conforme mostrado em [LW10], tal função pode ser representada por

$$
G(z, w)=-\frac{1}{2 \pi} \ln \left|\vartheta_{1}(z-w)\right|+\frac{1}{2 b}(\operatorname{Im}(z-w))^{2}+C(\tau)
$$

onde

$$
\vartheta_{1}(z ; \tau)=-i \sum_{-\infty}^{\infty}(-1)^{n} q^{\left(n+\frac{1}{2}\right)^{2}} e^{(2 n+1) \pi i z}
$$

é a função $\vartheta_{1}$ de Jacobi, $q=e^{\pi i \tau}$ e $C(\tau)$ é uma constante que só depende de $\tau$ e portanto não tem relevância para a construção da função de corrente. Assim sendo, dado um sistema de vórtices representado pela medida $\sum_{k=1}^{n} \Gamma_{k} \delta_{z_{k}}(z)$, a menos de uma constante que depende de $\tau$, a função de corrente correspondente é

$$
\psi(z)=\sum_{k=1}^{n} \Gamma_{k} G\left(z, z_{k}\right)=-\frac{1}{2 \pi} \sum_{k=1}^{n} \Gamma_{k}\left[\ln \left|\vartheta_{1}\left(z-z_{k} ; \tau\right)\right|-\frac{\pi}{b}\left(\operatorname{Im}\left(z-z_{k}\right)\right)^{2}\right] .
$$

Para determinarmos a equação de movimento para este sistema, devemos calcular a função de Robin no toro obtida pela regularização da função de Green na diagonal, processo do qual concluímos que o resultado é uma constante ${ }^{2}$, conforme nos mostra os cálculos seguintes

$$
m(z)=\lim _{w \rightarrow z}\left(G(z, w)+\frac{1}{2 \pi} \ln |z-w|\right)=-\frac{1}{2 \pi} \lim _{|\tilde{z}| \rightarrow 0} \ln \left(\frac{\left|\vartheta_{1}(\tilde{z})\right|}{|\tilde{z}|}\right)=\text { cte. }
$$

Considerando o resultado do capítulo 3 , temos que a função hamiltoniana para um sistema de dois vórtices é

$$
H\left(z_{1}, z_{2}\right)=-\frac{\Gamma_{1} \Gamma_{2}}{2 \pi}\left[\ln \left|\vartheta_{1}\left(z_{1}-z_{2} ; \tau\right)\right|-\frac{\pi}{b}\left(\operatorname{Im}\left(z_{1}-z_{2}\right)\right)^{2}\right],
$$

sendo as equações de movimento representadas por

$$
\Gamma_{1} \dot{z}_{1}=-J \nabla_{z_{1}} H \equiv-2 i \partial_{\bar{z}_{1}} H \text { e } \Gamma_{2} \dot{z}_{2}=-J \nabla_{z_{2}} H \equiv-2 i \partial_{\bar{z}_{2}} H .
$$

Definindo a posição relativa como sendo a nova variável $z:=z_{1}-z_{2}$, temos que $\partial_{\bar{z}_{1}}=\partial_{\bar{z}}$ e $\partial_{\bar{z}_{2}}=-\partial_{\bar{z}}$, donde segue-se

$$
\Gamma_{1} \dot{z}_{1}=-2 i \partial_{\bar{z}} H \text { e } \Gamma_{2} \dot{z}_{2}=2 i \partial_{\bar{z}} H
$$

Multiplicando a primeira equação em 5.5 por $\Gamma_{2}$, a segunda equação por $\Gamma_{1}$ e tomando a diferença, encontramos a seguinte equação diferencial para a posição relativa

$$
\dot{z}=-2 i \frac{\Gamma}{\Gamma^{*}} \partial_{\bar{z}} H(z ; \bar{z})=-2 i \Gamma \partial_{\bar{z}} G(z ; \bar{z}),
$$

onde $\Gamma=\Gamma_{1}+\Gamma_{2}, \Gamma^{*}=\Gamma_{1} \Gamma_{2}$ e $G=H / \Gamma^{*}$. A solução $z(t)$ desta última equação, com condição inicial adequada, nos permite resolver o sistema 5.5 por quadratura, conforme mostra as expressões abaixo

\footnotetext{
${ }^{2}$ Também podemos concluir este fato através de um argumento geométrico[Ste05]: a função de Robin numa superfície fechada é um invariante isométrico. Portanto, se o grupo de isometrias da superfície for transitivo a função de Robin deve ser constante.
} 


$$
\begin{aligned}
& z_{1}(t)=\int_{0}^{t}-\frac{2 i}{\Gamma_{1}} \partial_{\bar{z}} H(z(s) ; \bar{z}(s)) d s+z_{10} \\
& z_{2}(t)=\int_{0}^{t} \frac{2 i}{\Gamma_{2}} \partial_{\bar{z}} H(z(s) ; \bar{z}(s)) d s+z_{20}
\end{aligned},
$$

onde $z_{10}=z_{1}(0)$ e $z_{20}=z_{2}(0)$. No caso de vorticidade de fundo nula, ou seja, $\Gamma=0$, verifica-se que os vórtices deslocam-se ao longo de retas. De fato, $\Gamma=0$ implica $\dot{z}=0$, ou seja, $z=z(0)=$ $z_{10}-z_{20}=: z_{0}$ e portanto

$$
\begin{aligned}
& z_{1}(t)=-\frac{2 i}{\Gamma_{1}} \partial_{\bar{z}_{0}} H\left(z_{0} ; \bar{z}_{0}\right) t+z_{10} \\
& z_{2}(t)=\frac{2 i}{\Gamma_{2}} \partial_{\bar{z}_{0}} H\left(z_{0} ; \bar{z}_{0}\right) t+z_{20}
\end{aligned}
$$

A partir deste ponto, assumimos sempre que $\Gamma \neq 0$.

Definindo a re-escala de tempo dada pela aplicação $t \mapsto f \tau$, onde $f$ é uma constante, temos

$$
\dot{z}=-2 i \Gamma f \partial_{\bar{z}} \tilde{H} .
$$

Portanto, dado que $\Gamma \neq 0$, podemos escolher $f=1 / \Gamma$ e escrever a equação de evolução da posição relativa $z$ da seguinte forma

$$
\dot{z}=-2 i \partial_{\bar{z}} G .
$$

A equação 5.8 nos mostra que a posição relativa desloca-se ao longo das curvas de nível da função de Green para o Laplaciano no toro. Definindo o centro de vorticidade do sistema pela expressão

$$
w=\frac{\Gamma_{1}}{\Gamma} z_{1}+\frac{\Gamma_{2}}{\Gamma} z_{2},
$$

o resultado apresentado na equação 5.5 nos diz que $\Gamma_{1} z_{1}=-\Gamma_{2} z_{2}$ e portanto é imediato verificar a conservação do centro de vorticidade, isto é, $\dot{w}=\mathbf{0}$. Como $z(t)=z_{1}(t)+z_{2}(t) \mathrm{e}$

$$
\frac{\Gamma_{1}}{\Gamma} z_{1}(t)+\frac{\Gamma_{2}}{\Gamma} z_{2}(t)=w(t)=w(0)=\frac{\Gamma_{1}}{\Gamma} z_{10}+\frac{\Gamma_{2}}{\Gamma} z_{20},
$$

sendo $z_{10}=z_{1}(0)$ e $z_{20}=z_{2}(0)$, com uma simples manipulação algébrica obtemos as seguintes expressões

$$
z_{1}=w+\frac{\Gamma_{2}}{\Gamma} z \text { e } z_{2}=w-\frac{\Gamma_{1}}{\Gamma} z .
$$

Estas equações nos dizem que toda informação sobre a dinâmica de um sistema de dois vórtices no toro plano pode ser inferida a partir da evolução temporal da posição relativa entre eles. De fato, nota-se que as trajetórias $z_{1}(t)$ e $z_{2}(t)$ são obtidas a partir da trajetória $z(t)$ através da composição de uma homotetia com uma translação. Assim sendo, as órbitas dos vórtices terão o mesmo tipo topológico da órbita $z$ e, se esta for fechada, $z_{1}$ e $z_{2}$ serão fechadas e de mesmo período. Também nota-se que os equilíbrios relativos deste sistema são equilíbrios absolutos e correspondem a pontos de equilíbrios da posição relativa que, por sua vez, são selas ou centros da hamiltoniana $G$. Daí, todo estudo sobre estabilidade, bifurcação e perturbação de um sistema de dois vórtices no toro plano pode ser desenvolvido fazendo a análise do problema correspondente na variável $z$, que, por sua vez, reduz-se ao estudo da hamiltoniana $G$. Na próxima seção faremos um estudo numérico das curvas de nível da função $G$ e suas bifurcações.

\subsubsection{Bifurcações das curvas de nível da função de Green no toro plano}

Para desenvolvermos o trabalho apresentado nesta seção, utilizamos os resultados do artigo [LW10] que trata de um estudo analítico dos equilíbrios da função de Green no toro plano e suas implicações no estudo das soluções da equação de campo médio nesta superfície. Nosso interesse é compreender a topologia das curvas de nível de $G$, o que está relacionado, essencialmente, aos tipos de pontos críticos desta função, conexões homoclínicas e heteroclínicas, bem como à combinatória gerada por estes elementos e sua bifurcação com respeito à variação do parâmetro modular $\tau$. 
Considere um toro $T=\mathbb{C} / \Lambda$ com reticulado $\Lambda=(\mathbb{Z}+\mathbb{Z} \tau)$ onde $\tau=a+b i$ com $b>0$ e definamos $q=e^{\pi i \tau}$ onde $|q|=e^{-\pi b}<1$. Como apresentado na seção anterior, a função de Green para o laplaciano no toro $T$ pode ser escrita em termos da função $\vartheta_{1}$ de Jacobi, sendo sua expressão mais explícita dada por[SA00]:

$$
G(x, y, a, b)=-\frac{1}{2 \pi}\left\{\ln \left[\left|\frac{\vartheta_{1}\left(x+i y ; e^{\pi i(a+b i)}\right]}{\left.\vartheta_{1}\left[0 ; e^{\pi i(a+b i)}\right]\right)}\right|\right]-\frac{\pi y^{2}}{b}\right\} .
$$

Esta função define a hamiltoniana para posição relativa de dois vórtices em $T$ com vorticidades não nulas e cuja soma também é não nula. O comportamento qualitativo deste sistema depende dos valores dos parâmetros $a$ e $b$ que definem o reticulado $\Lambda$, de modo que a variação destes parâmetros controla o surgimento de singularidades e a mudança no padrão das separatrizes. Note que como $b>0$, o parâmetro modular $\tau$ é um elemento do semi-plano superior $\mathbb{H}:=\left\{(a, b) \in \mathbb{R}^{2} \mid b>0\right\}$.

\section{Descrição das bifurcações dos equilíbrios}

Um fato que facilita a análise aqui apresentada é que a função de Green para o laplaciano no toro plano possui 3 ou 5 singularidades[LW10], as quais correspondem aos possíveis pontos de equilíbrios da equação 5.8. Três destes equilíbrios são óbvios, considerando que os meio períodos são sempre singularidades da função de Green. De fato, como a função de Green é par, tem-se que $\nabla G(z)=-\nabla G(-z)$ e portanto, avaliando em um meio período $w$, segue-se $\nabla G(w)=-\nabla G(-w)=$ $-\nabla G(w)$, pois no toro vale que $G(w)=G(-w)$ quando $w$ é um meio período. Daí resulta que $\nabla G(w)=\mathbf{0}$, isto é, um meio-período é equilíbrio da função de Green. Com isto, para qualquer valor do parâmetro modular $\tau$, temos sempre três equilíbrios dados pelos meio-períodos

$$
w_{1}=1 / 2, w_{2}=\tau / 2 \text { e } w_{3}=1 / 2+\tau / 2 .
$$

Considerando a compacidade do toro, o índice das singularidades e o fato destas serem em número de três ou cinco, além do vórtice que é topologicamente um centro, o surgimento de novos equilíbrios ocorre com a degenerescência de um dos meio-períodos, o qual bifurca de centro para sela com o surgimento de mais dois centros. Apresentaremos, nesta seção, os diagramas de bifurcação para o surgimento destes equilíbrios, onde o espaço dos parâmetros é o semiplano superior com coordenadas $(a, b), b>0$. Apresentamos também o retrato de fase da equação 5.8 para alguns pontos representativos no espaço dos parâmetros. Os diagramas de bifurcação para os equilíbrios são feitos a partir da expressão analítica da condição de degenerescência, ou seja, apresentando graficamente as soluções numéricas das equações implícitas $\operatorname{Hess}(G)\left(w_{i} ; a, b\right) \equiv 0, i=1,2,3$, onde $\operatorname{Hess}(G)=\left(\partial_{x x} G\right)\left(\partial_{y y} G\right)-\left(\partial_{x y} G\right)^{2}$ representa a hessiana da função de Green. Denotamos por $C D_{k}$ a curva de degenerescência do meio-período $w_{k}$, definida por

$$
C D_{k}:=\left\{(a, b) \in \mathbb{H} \mid \operatorname{Hess}(G)\left(w_{k} ; a, b\right) \equiv 0\right\}, k=1,2,3 .
$$

Os resultados obtidos são apresentados na figura 5.6, onde, em virtude da periodicidade, os gráficos de $C D_{1}, C D_{2}$ e $C D_{3}$ são elaborados para $a$ pertencente ao intervalo $(-1,1)$. Note que $\operatorname{Hess}(G)(z ; a+$ $2, b)=\operatorname{Hess}(G)(z ; a, b)$, ou seja, o diagrama de bifurcação tem periodicidade 2 com respeito ao parâmetro $a$. Além disso, verifica-se que a curva de degenerescência de $w_{1}$ tem simetria com respeito às reflexões com relação às retas verticais definidas por $a=\frac{k}{2}, \quad k \in \mathbb{Z}$, o que pode ser assinalado pela invariância em relação à ação $(a, b) \mapsto(k-a, b), \quad k \in \mathbb{Z}$. Já as curvas de $C D_{2}$ e $C D_{3}$ são invariantes com respeito às reflexões em relação às retas verticais de abscissa inteira, donde seguese que o diagrama é invariante pelo mapa $(a, b) \mapsto(2 k-a, b), \quad k \in \mathbb{Z}$. Desta forma, para análise de bifurcação basta considerarmos o diagrama no domínio $a \in(0,1)$ e $b \in(0,1)$. Estas simetrias estão relacionadas com a escolha de diferentes bases para um mesmo reticulado correspondente a um toro e em termos da ação de uma rotação de $180^{\circ}$ sobre um reticulado normalizado, questões que serão abordadas no final deste capítulo. Nota-se também a não interseção de quaisquer duas destas curvas de degenerescência, o que revela o fato já esperado de que os equilíbrios adicionais 


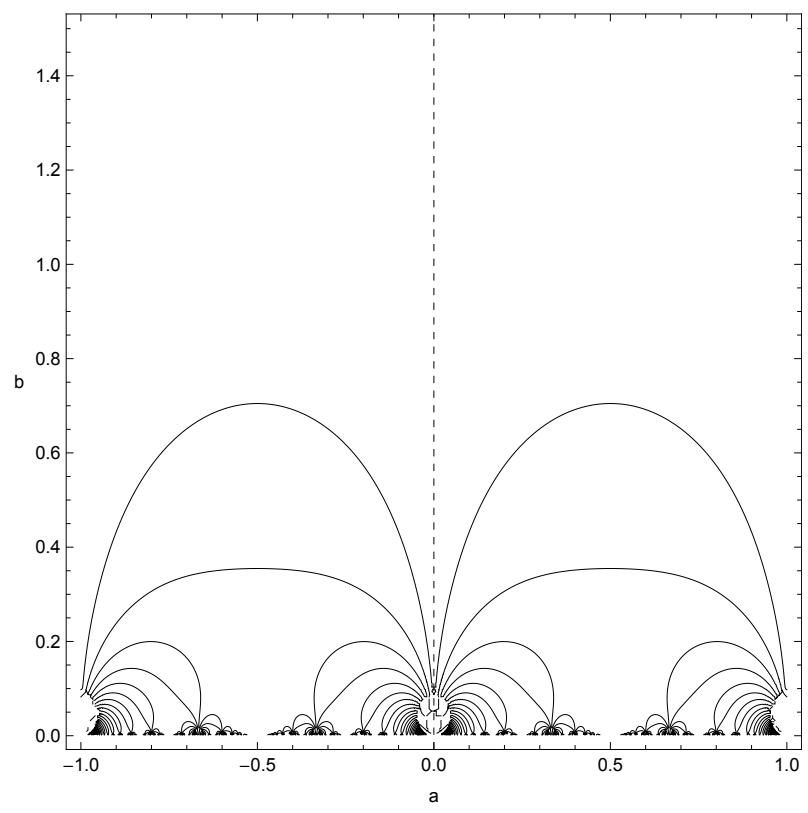

a) Gráfico do conjunto $C D_{1}$

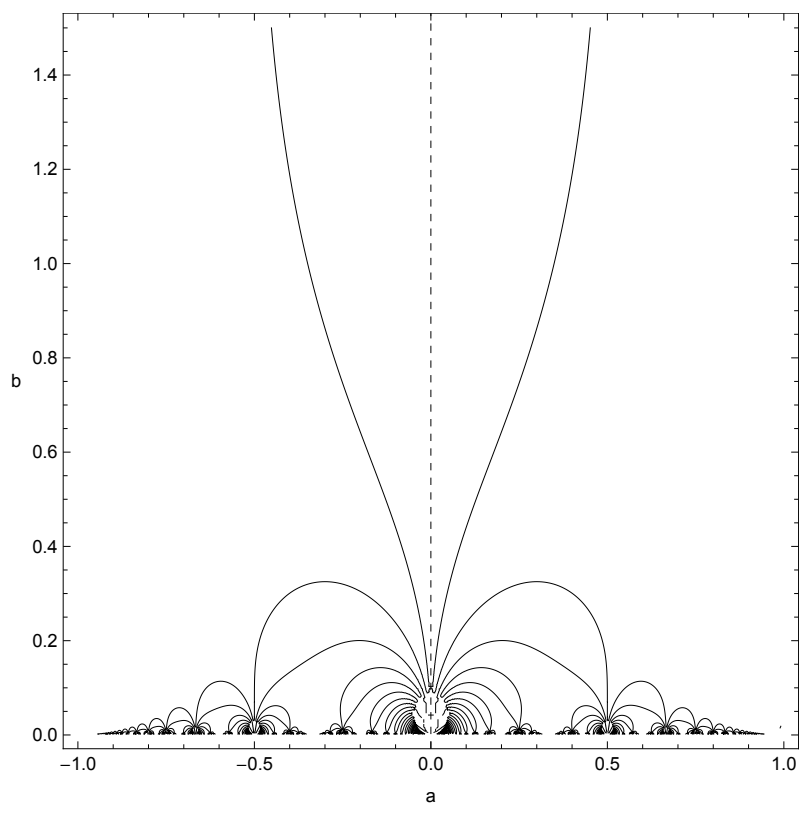

c) Gráfico do conjunto $C D_{3}$

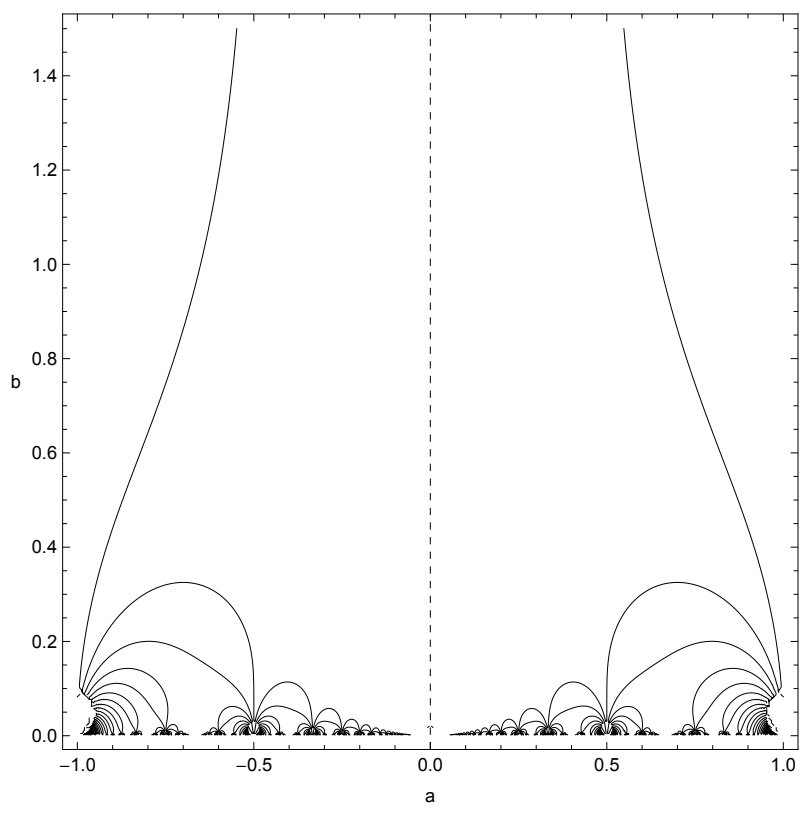

b) Gráfico do conjunto $C D_{2}$

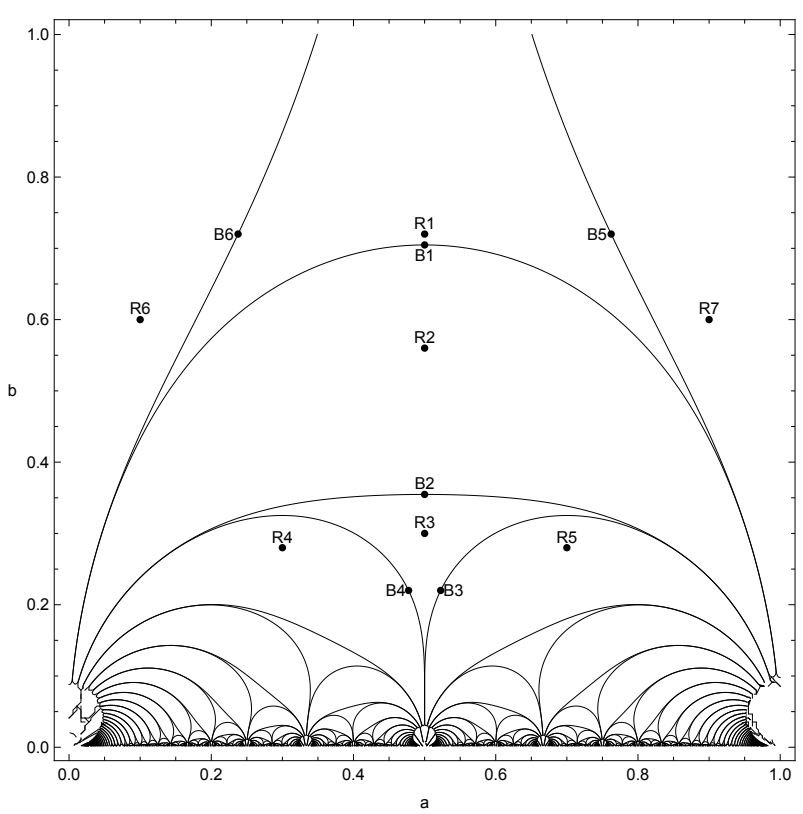

d) Diagrama de bifurcação dos equilíbrios onde são representados os pontos escolhidos para análise

Figura 5.6: a) conjunto de degenerescência de $\left.w_{1} ; b\right)$ conjunto de degenerecência de $\left.w_{2} ; c\right)$ conjunto de degenerescência de $w_{3} ;$ d) representação do diagrama de bifurcação dos equilíbrios. 
bifurcam a partir de um mesmo meio-período. Para representarmos os retratos de fase, escolhemos cinco pontos de bifurcação rotulados por $B_{1}, \cdots, B_{5}$ e sete pontos regulares $R_{1}, \cdots, R_{7}$, conforme ilustrado na figura $5.6 \mathrm{~d}$ ). A seguir, apresentamos os retratos de fase de cada um destes pontos.

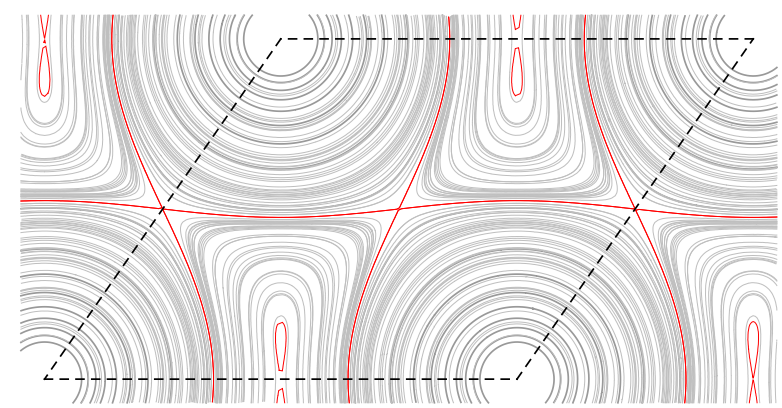

Curvas de nível: ponto $R 1$

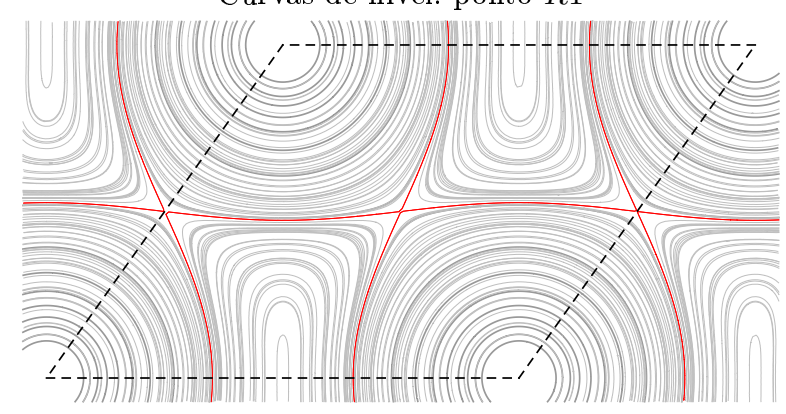

Curvas de nível: ponto $B 1$

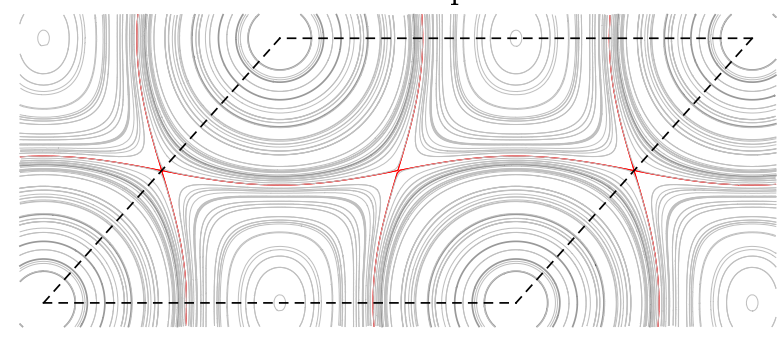

Curvas de nível: ponto $R 2$

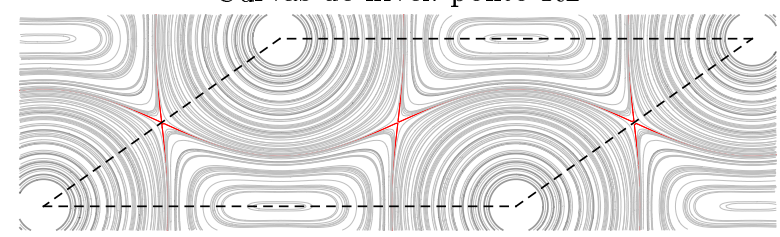

Curvas de nível: ponto $B 2$

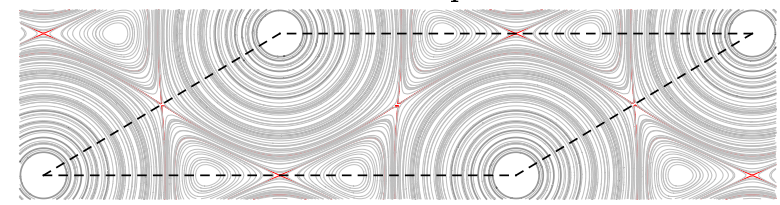

Curvas de nível: ponto $R 3$

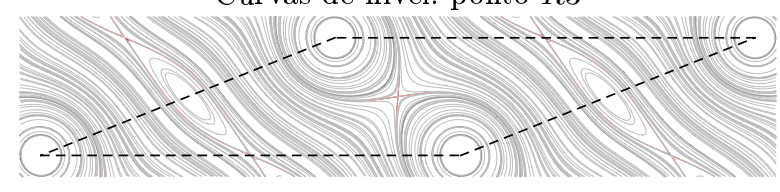

Curvas de nível: ponto $R 5$

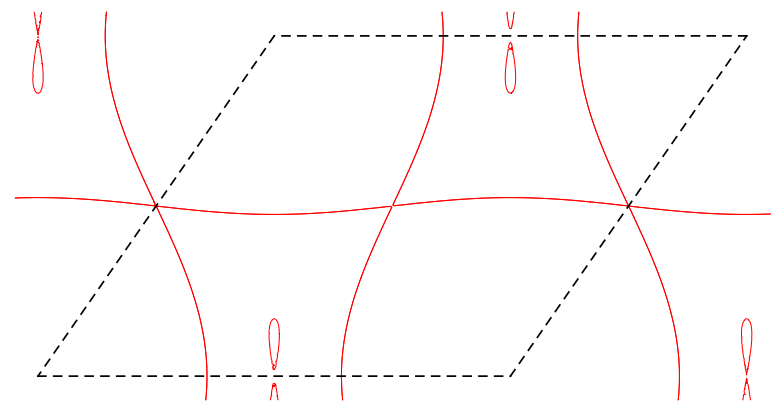

Separatrizes: ponto $R 1$

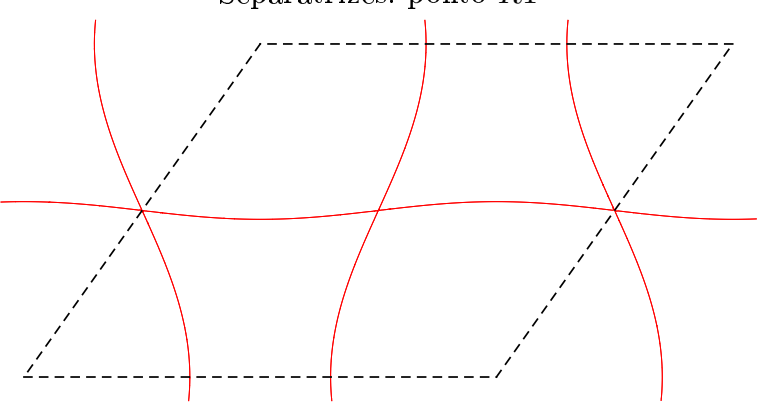

Separatrizes: ponto $B 1$

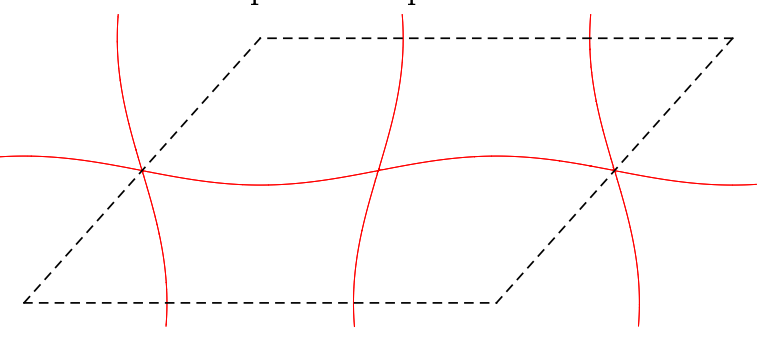

Separatrizes: ponto $R 2$

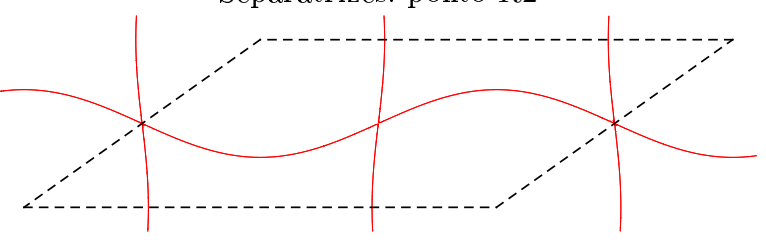

Separatrizes: ponto $B 2$

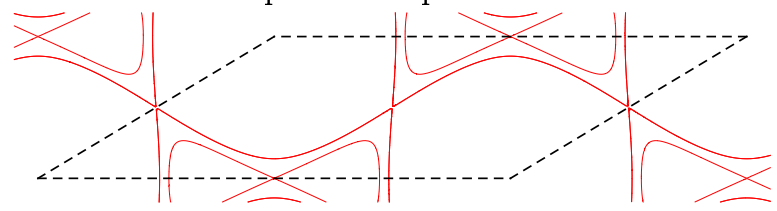

Separatrizes: ponto $R 3$

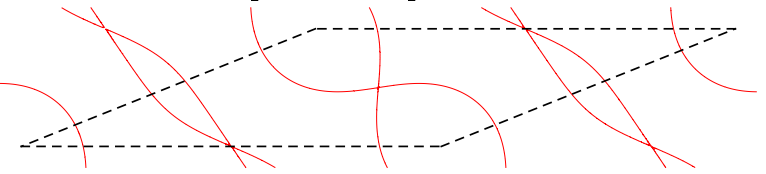

Separatrizes: ponto $R 5$

Figura 5.7: Retratos de fase correspondentes aos pontos $R 1, B 1, R 2, B 2, R 3$ e $R 5$. 


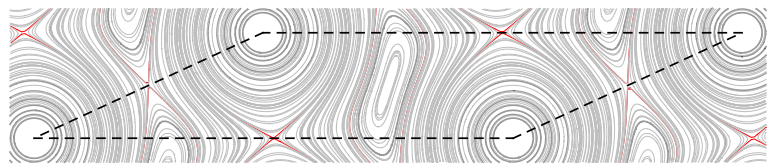

Curvas de nível: ponto $B 4$

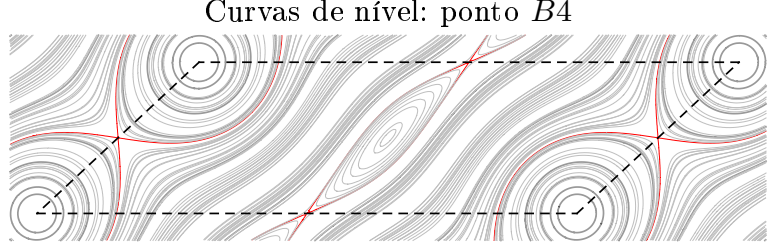

Curvas de nível: ponto $R 4$

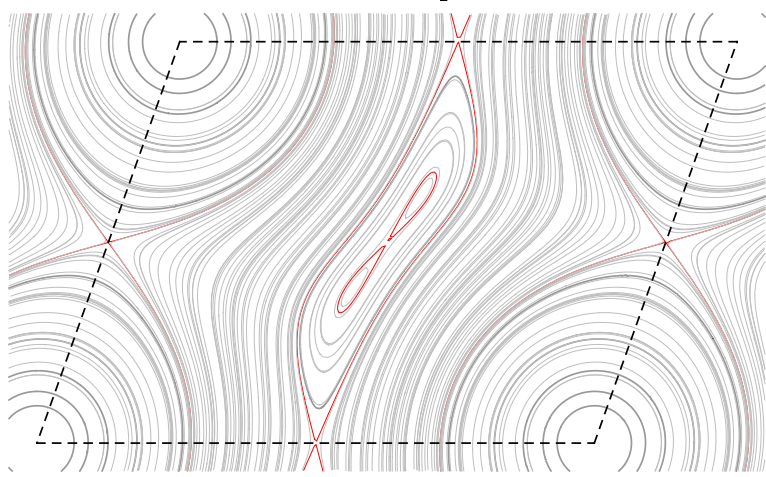

Curvas de nível: ponto $B 6$

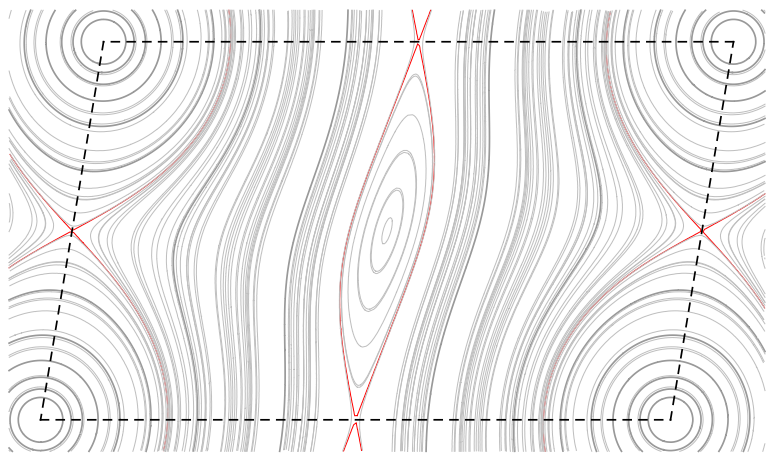

Curvas de nível: ponto $R 6$

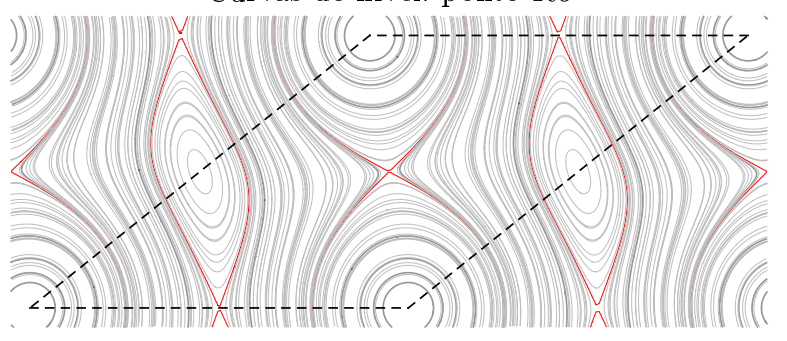

Curvas de nível: ponto $B 5$

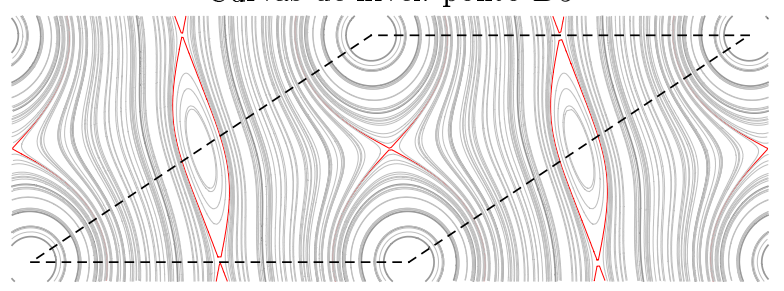

Curvas de nível: ponto $R 7$

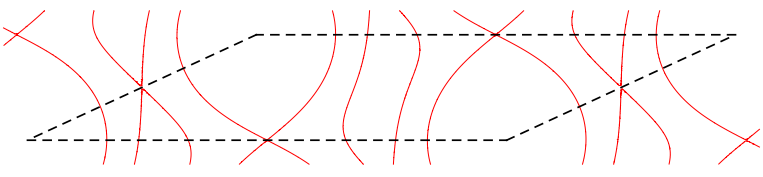

Curvas de nível: ponto $B 4$

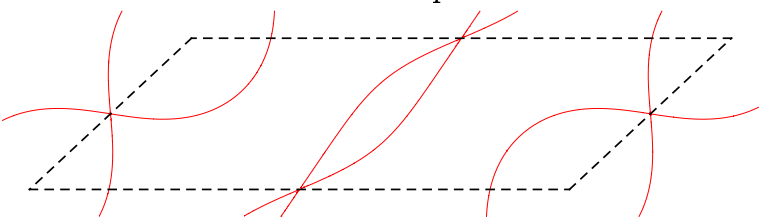

Separatrizes: ponto $R 4$

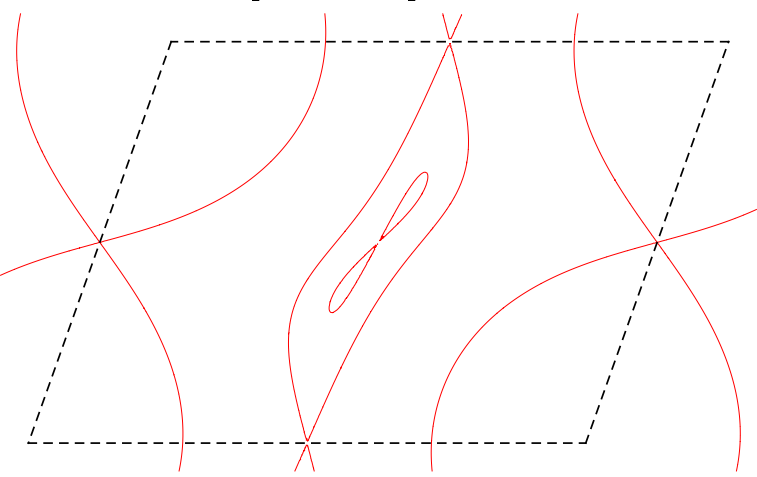

Separatrizes: ponto $B 6$

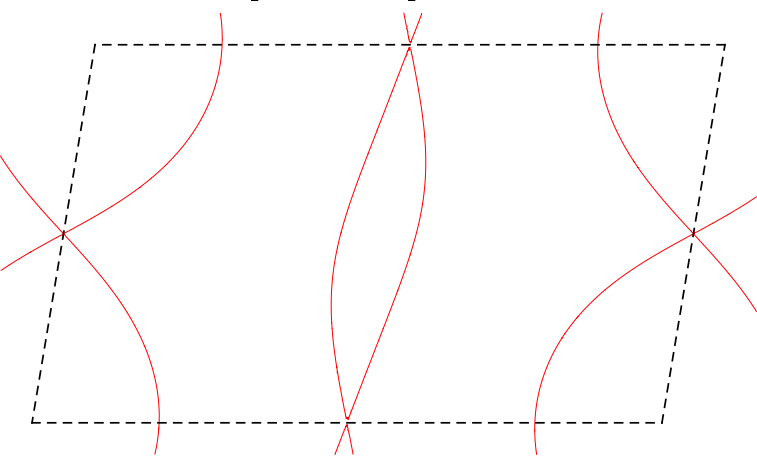

Separatrizes: ponto $R 6$

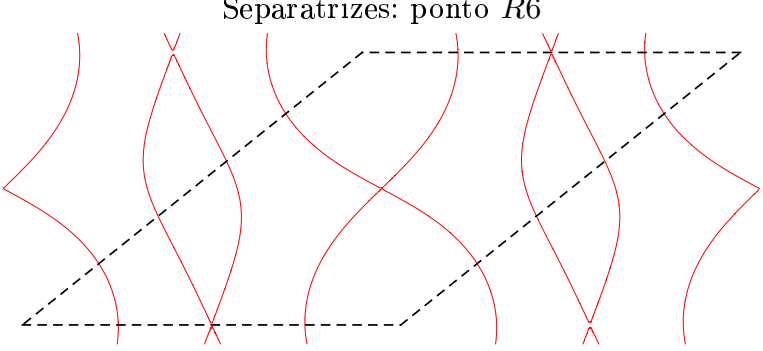

Separatrizes: ponto $B 5$

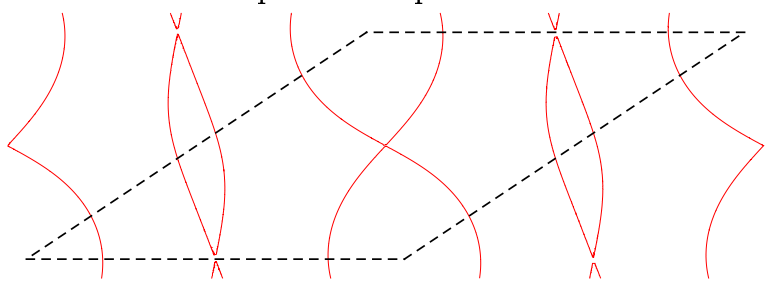

Separatrizes: ponto $R 7$

Figura 5.8: Retratos de fase correspondentes aos pontos $B 4, R 4, B 6, R 6, B 5$ e $R 7$. 


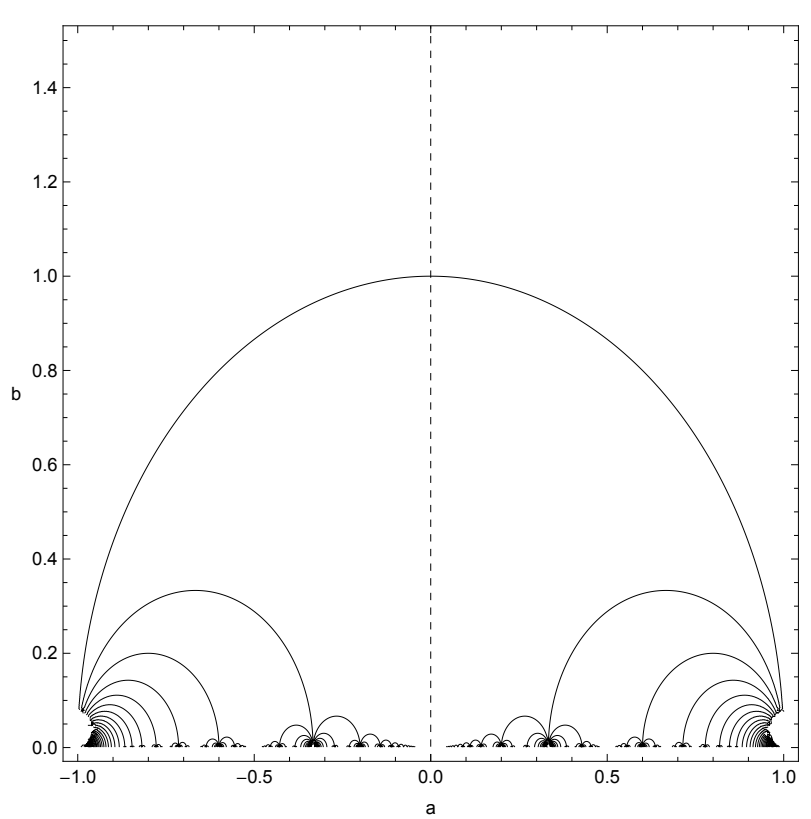

a) Gráfico do conjunto $C_{12}$

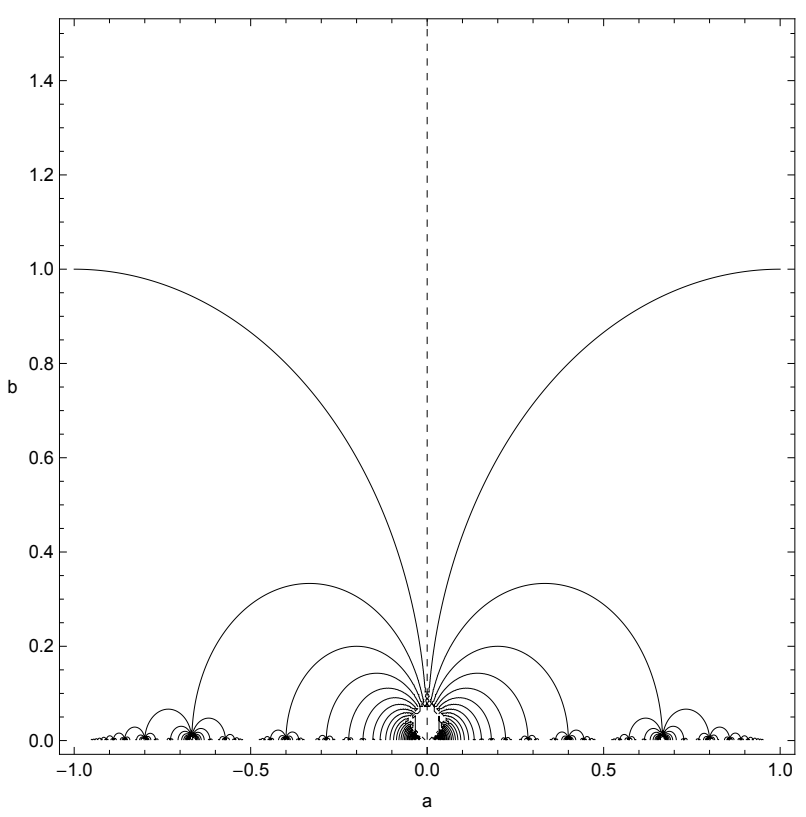

b) Gráfico do conjunto $C_{13}$

Figura 5.9: Representação dos pontos para os quais ocorre conexão entre: a) $w_{1}$ e $w_{2}$; b) $w_{1}$ e $w_{3}$

\section{Descrição das bifurcações das separatrizes}

Sabe-se que os equilíbrios para a equação da posição relativa $z$ são centros ou selas. Portanto, as bifurcações globais devem ocorrer com a mudança no padrão das separatrizes de sela, isto é, criação e destruição de separatrizes homoclínicas e heteroclínicas conforme mudamos os valores do parâmetro modular $\tau$. Estas bifurcações são analiticamente detectadas quando a hamiltoniana assume o mesmo valor em dois meio-períodos distintos. Assim, conforme seja o caso, devemos representar graficamente a solução numérica das equações $G\left(w_{i} ; a, b\right)-G\left(w_{j} ; a, b\right) \equiv 0, i, j=1,2,3$ onde $i \neq j$. A partir da expressão da função de Green, definimos as funções

$$
\begin{aligned}
& F_{12}(\tau):=\left|\vartheta_{1}(1 / 2 ; \tau)\right|-e^{\frac{-\pi b}{4}}\left|\vartheta_{1}(\tau / 2 ; \tau)\right| \\
& F_{13}(\tau):=\left|\vartheta_{1}(1 / 2 ; \tau)\right|-e^{\frac{-\pi b}{4}}\left|\vartheta_{1}\left(\frac{\tau+1}{2} ; \tau\right)\right| \\
& F_{23}(\tau):=\left|\vartheta_{1}(\tau / 2 ; \tau)\right|-\left|\vartheta_{1}(\tau / 2 ; \tau)\right|,
\end{aligned}
$$

de modo que $G\left(w_{i} ; \tau\right)-G\left(w_{j} ; \tau\right) \equiv 0$ se e somente se $F_{i j}(\tau) \equiv 0$ para $i, j=1,2,3$ com $i \neq j$ e $\tau=a+b i$. Denotamos por $C_{i j}$ os conjuntos definidos pelo nível zero das funções $F_{i j}$ :

$$
C_{i j}:=\left\{\tau \in \mathbb{H} \mid F_{i, j}(\tau) \equiv 0\right\},
$$

de modo que desejamos estudar a estrutura destes conjuntos no espaço dos parâmetros, uma vez que $C_{i j}$ descreve os valores dos parâmetros para os quais existe uma separatriz conectando os meioperíodos $w_{i}$ e $w_{j}$. Conforme observado nas figuras 5.9 e 5.10 , vê-se que os resultados obtidos revelam simetrias e periodicidade semelhantes às assinaladas no caso dos diagramas de degenerescência, fato natural e perfeitamente justificado em virtude da causa destas simetrias serem as mesmas e de natureza puramente geométrica, conforme será explicado no final deste capítulo.

Pelo próprio significado das curvas em análise, é claro que se duas delas intersectam-se em um ponto então as três intersectam-se neste ponto. Desta forma, os retratos de fase para parâmetros em uma vizinhança de tais pontos são qualitativamente representativos e significantes para um estudo das bifurcações, de modo que destacamos regiões numeradas de 1 a 4 correspondentes a quatro destes pontos e elaboramos os retratos de fase para pontos representativos escolhidos em cada uma destas regiões. Na figura 5.10 podemos observar o diagrama de bifurcação com as regiões nas quais serão selecionados pontos para descrevermos os retratos de fase correspondentes. Nas figuras 5.11, 


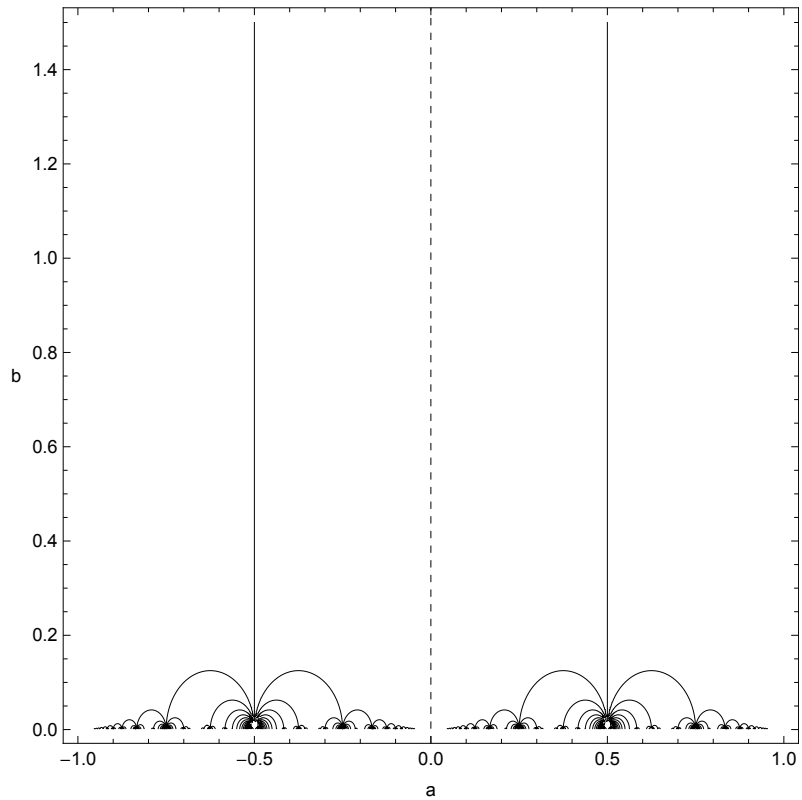

a) Gráfico do conjunto $C_{23}$

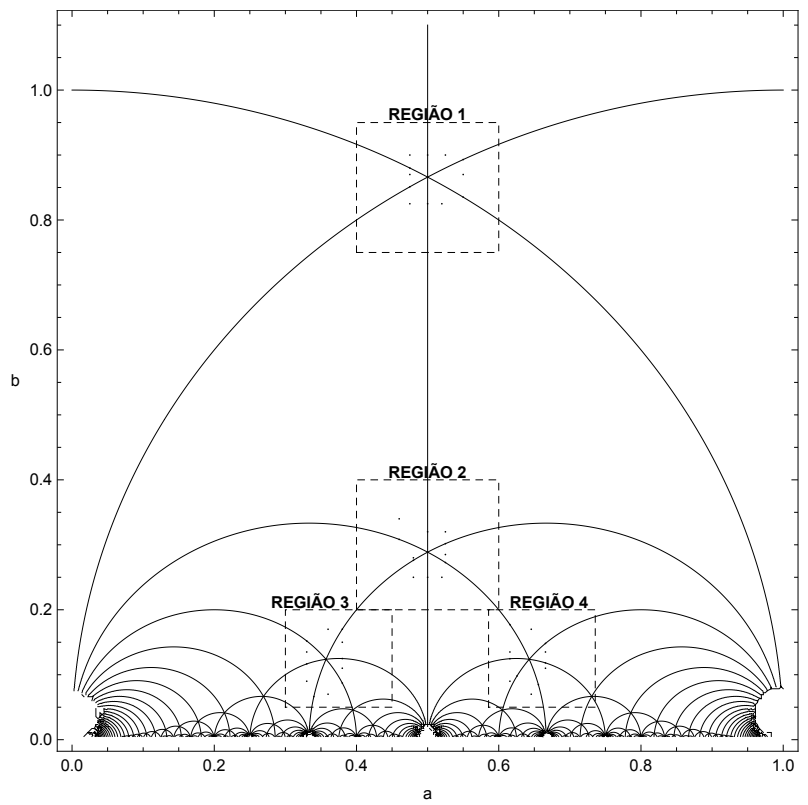

b) Diagrama de bifurcação das conexões

Figura 5.10: a) gráfico do conjunto $C_{23}$, o qual representa os pontos para os quais ocorre conexão entre os meio-periodos $w_{2}$ e $\left.w_{3} ; b\right)$ representação do diagrama de bifurcação das conexões, onde assinala-se as regiões selecionadas para análise do retrato de fase.

$5.13,5.15$ e 5.17 são apresentados os pontos escolhidos em cada uma das regiões. Note que estes pontos são identificados por uma sequência de um, dois ou três algarismos, sendo que o primeiro identifica a região ao qual pertence. Os pontos de interseção são identificados por um único algarismo enquanto que pontos com dois algarismos pertencem a um dos conjuntos $C_{i j}$. Pontos identificados por três algarismos são regulares para este diagrama e os dois últimos algarismos identificam que o mesmo pertence ao setor do espaço dos parâmetros delimitado pelas curvas correpondentes a estes algarismos. Vejamos agora os retratos de fase para cada uma das regiões. 


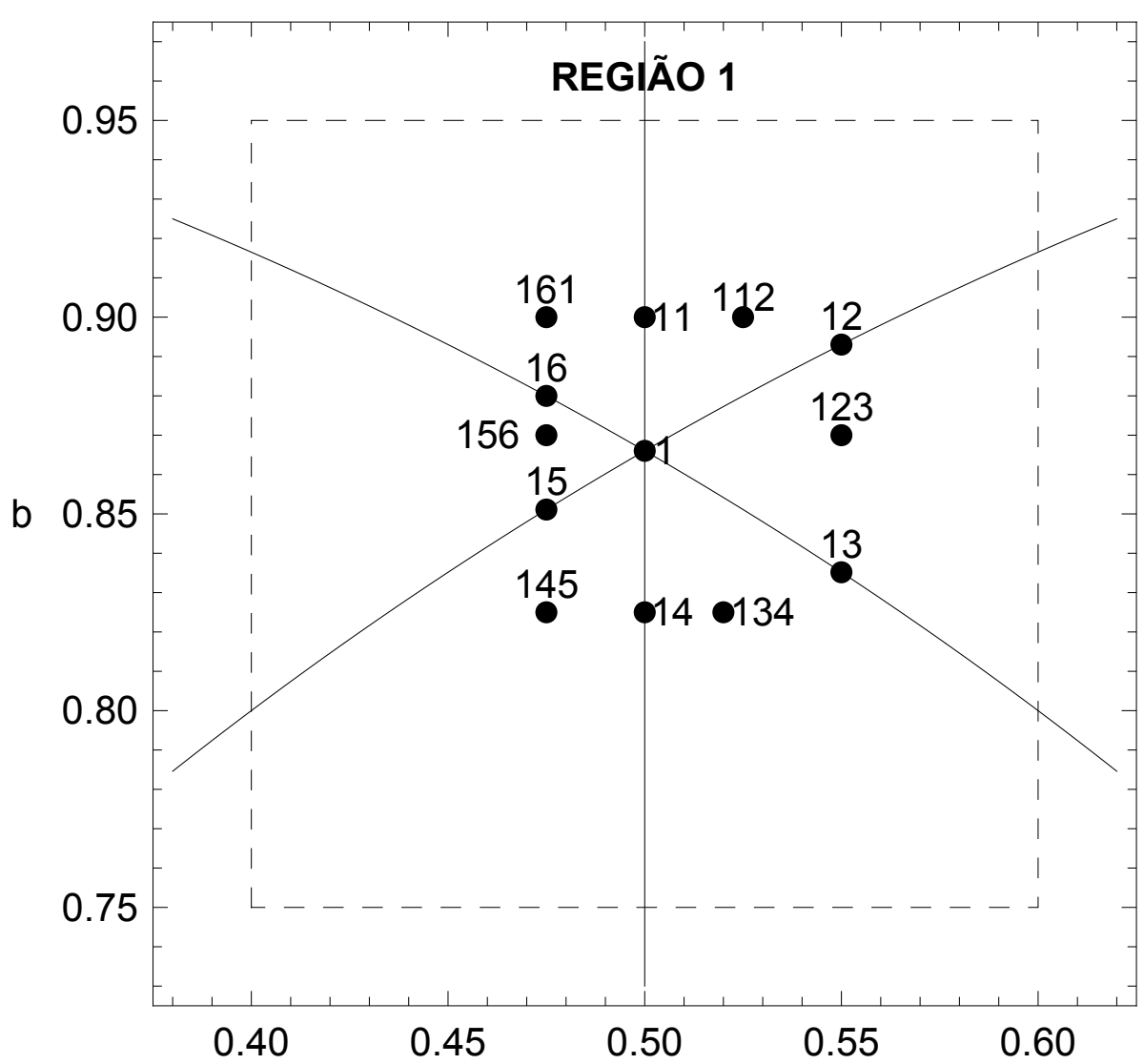

a

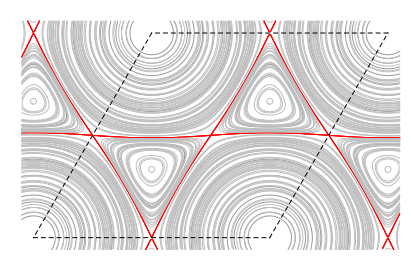

Curvas de nível: ponto 1

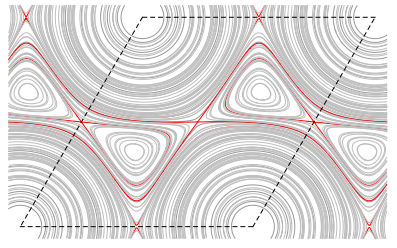

Curvas de nível: ponto 112

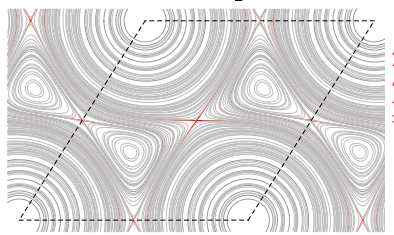

Curvas de nível: ponto 123

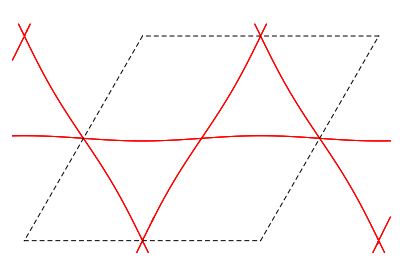

Separatrizes: ponto 1

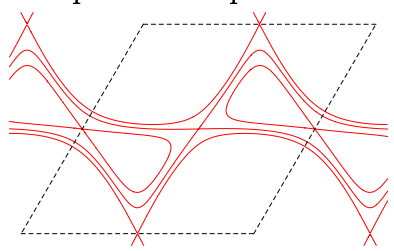

Separatrizes: ponto 112

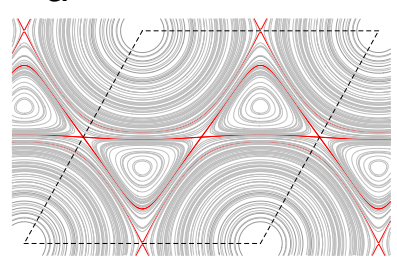

Curvas de nível: ponto 11

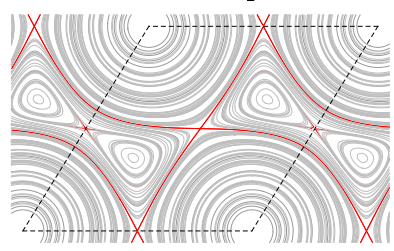

Curvas de nível: ponto 12

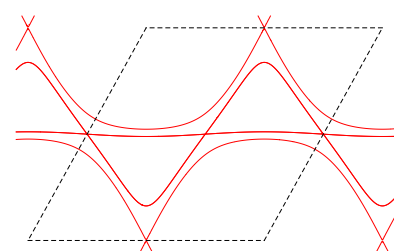

Separatrizes: ponto 11

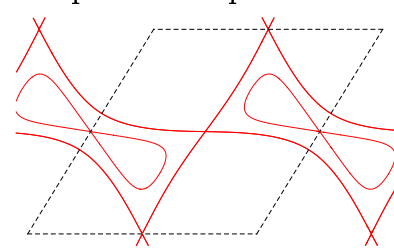

Separatrizes: ponto 12
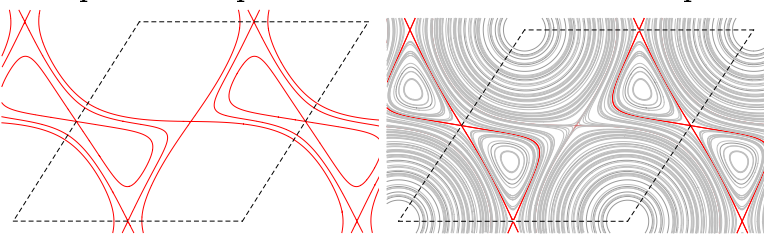

Curvas de nível: ponto 13

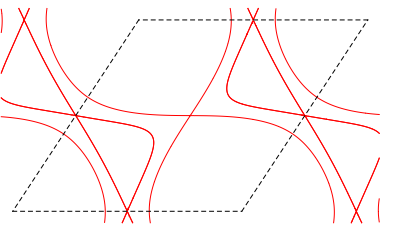

Separatrizes: ponto 13

Figura 5.11: Retratos de fase correspondentes aos pontos 1,11,112,12, 123 e 13 . 


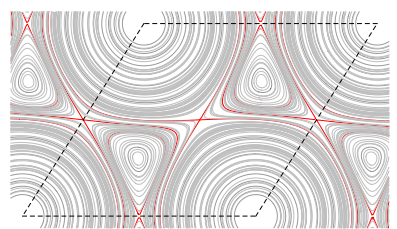

Curvas de nível: ponto 134

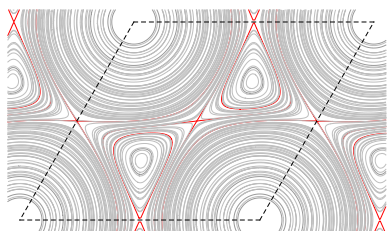

Curvas de nível: ponto 145

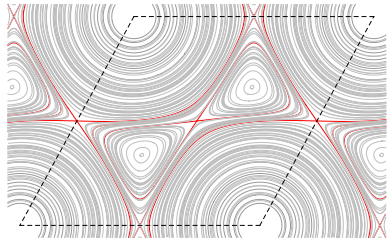

Curvas de nível: ponto 156

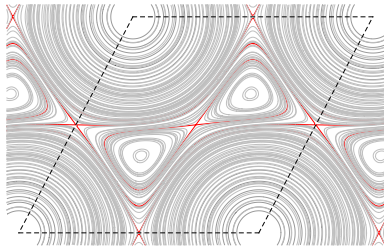

Curvas de nível: ponto 161

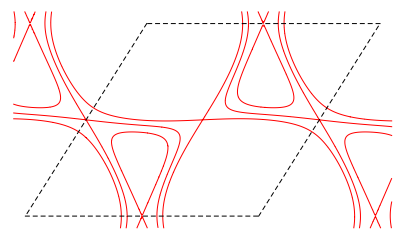

Separatrizes: ponto 134

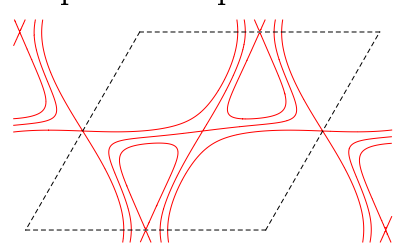

Separatrizes: ponto 145

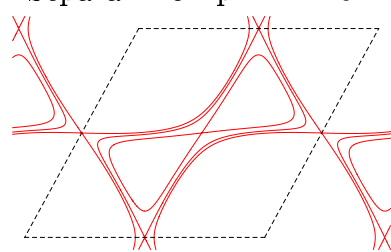

Separatrizes: ponto 156

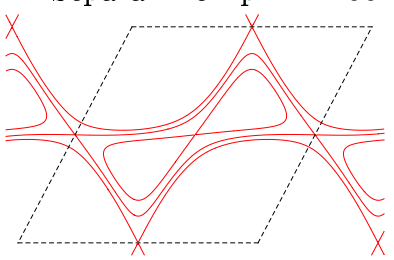

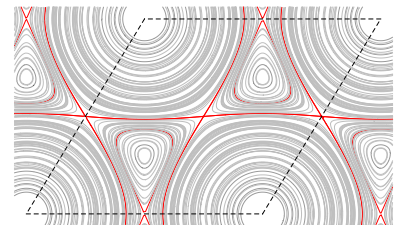

Curvas de nível: ponto 14

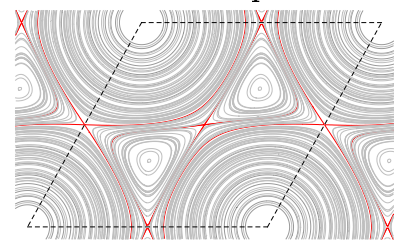

Curvas de nível: ponto 15

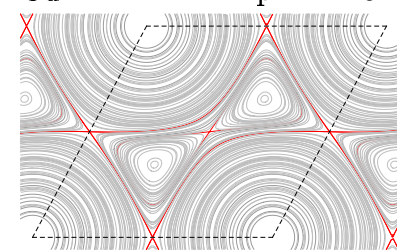

Curvas de nível: ponto 16

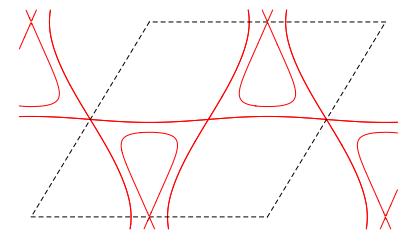

Separatrizes: ponto 14

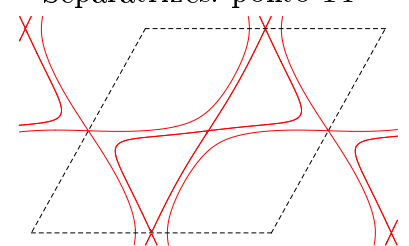

Separatrizes: ponto 15

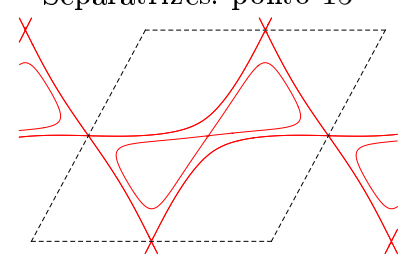

Separatrizes: ponto 16

Figura 5.12: Retratos de fase dos pontos 134, 14, 145, 15, 156, 16 e 161 . 


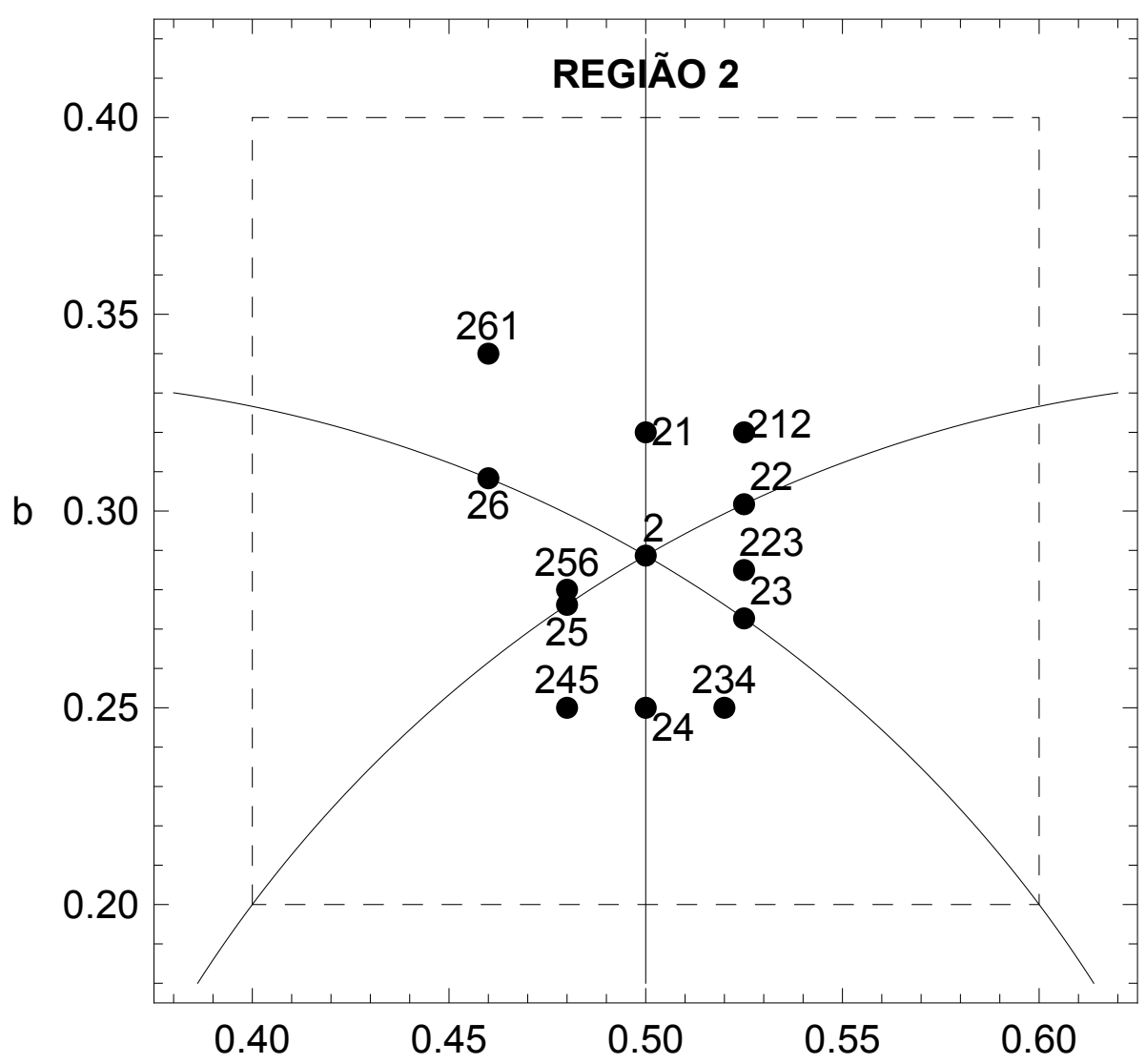

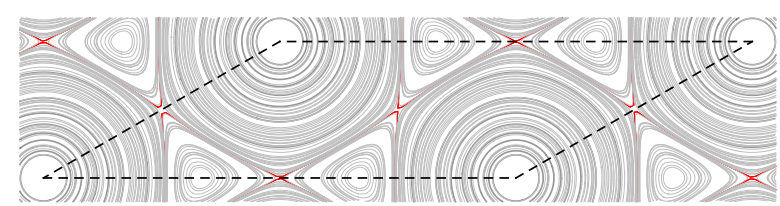

Curvas de nível: ponto 2

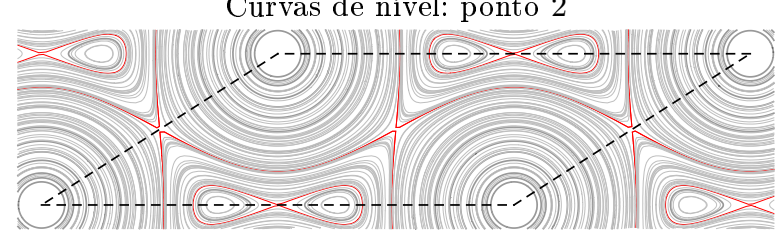

Curvas de nível: ponto 21

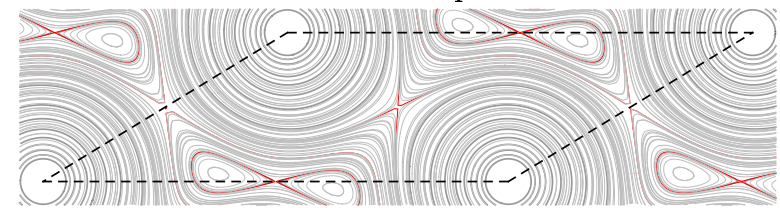

Curvas de nível: ponto 212

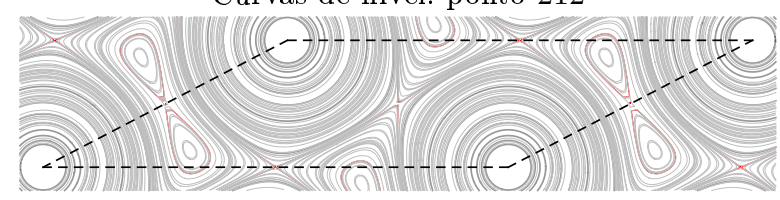

Curvas de nível: ponto 22

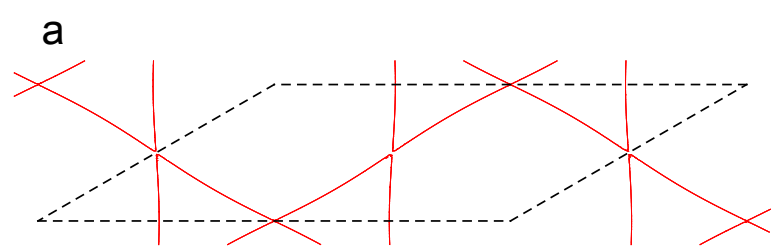

Separatrizes: ponto 2

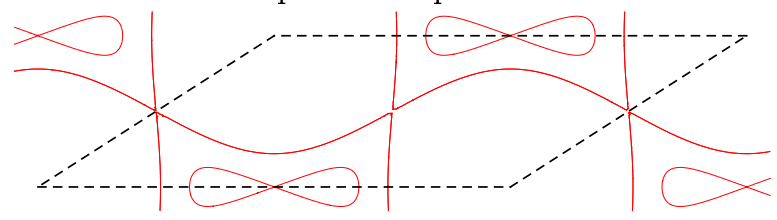

Separatrizes: ponto 21

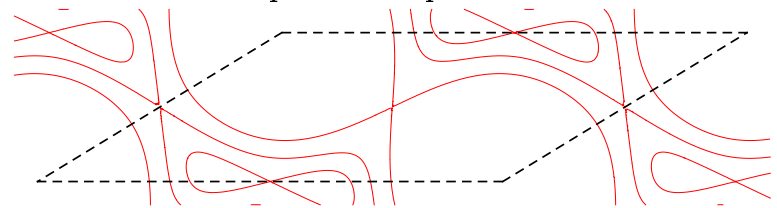

Separatrizes: ponto 212

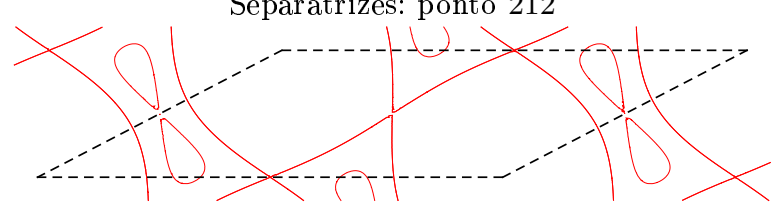

Separatrizes: ponto 22

Figura 5.13: Retratos de fase dos pontos 2,21, 212 e 22 . 


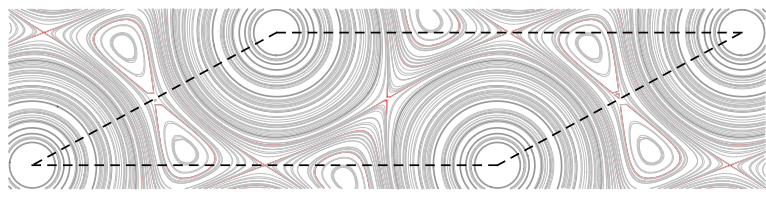

Curvas de nível: ponto 223

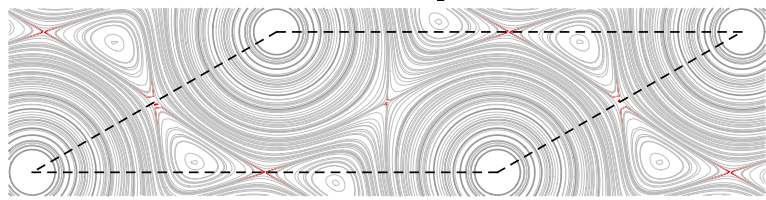

Curvas de nível: ponto 23

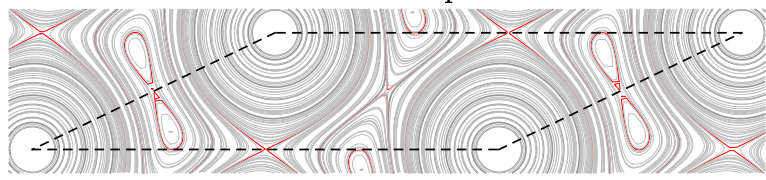

Curvas de nível: ponto 234

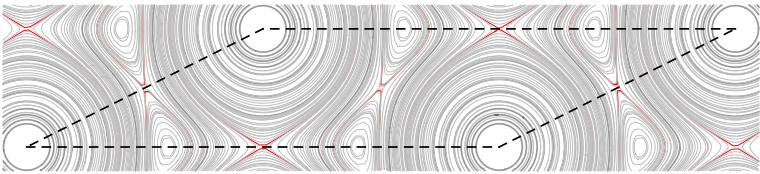

Curvas de nível: ponto 24

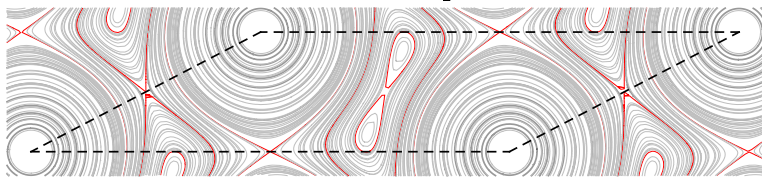

Curvas de nível: ponto 245

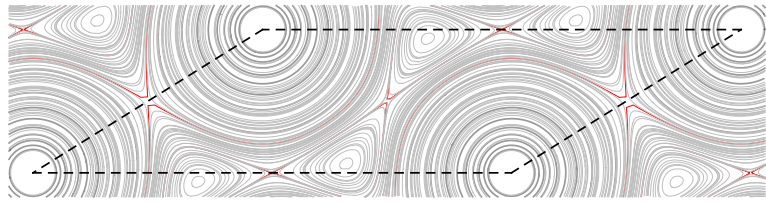

Curvas de nível: ponto 25

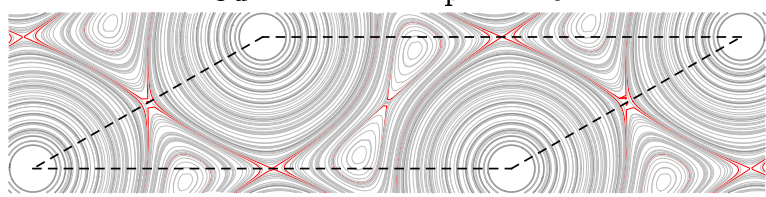

Curvas de nível: ponto 256

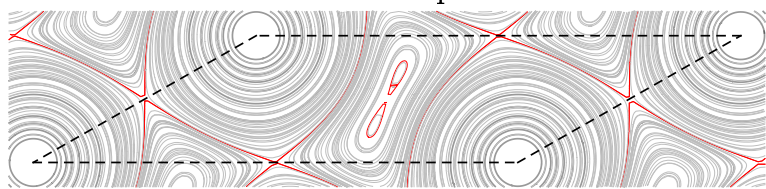

Curvas de nível: ponto 26

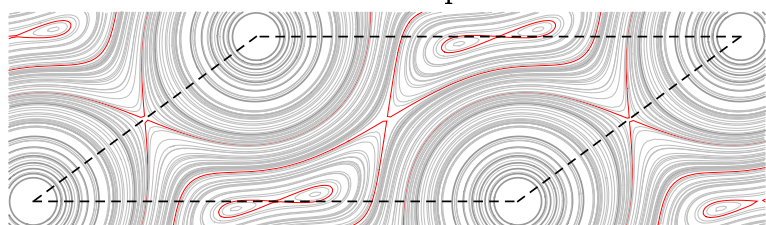

Curvas de nível: ponto 261

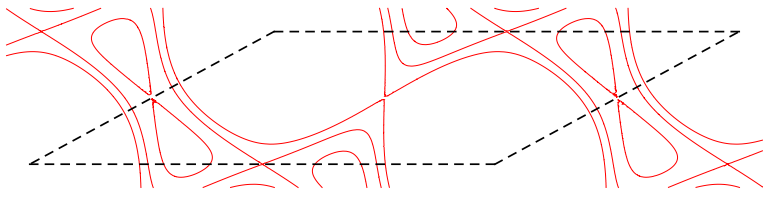

Separatrizes: ponto 223

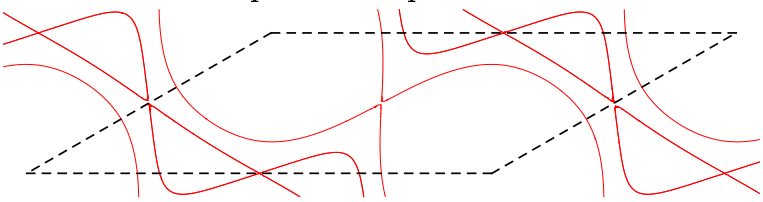

Separatrizes: ponto 23

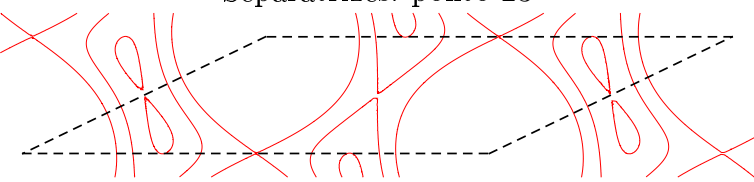

Separatrizes: ponto 234

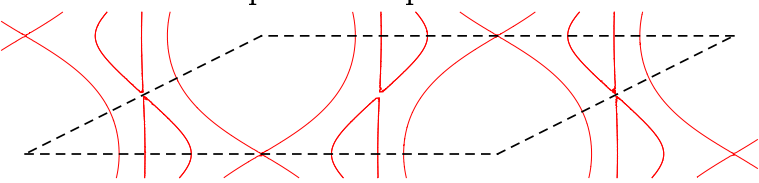

Separatrizes: ponto 24

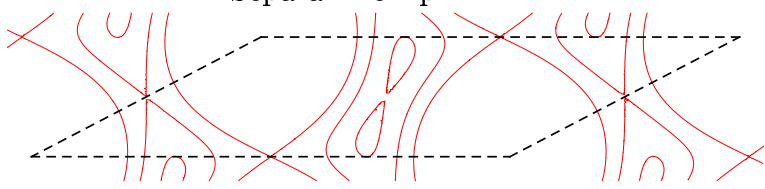

Separatrizes: ponto 245

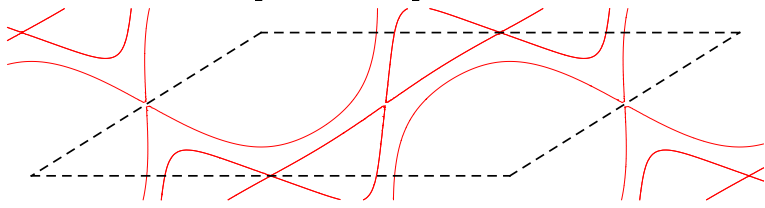

Separatrizes: ponto 25

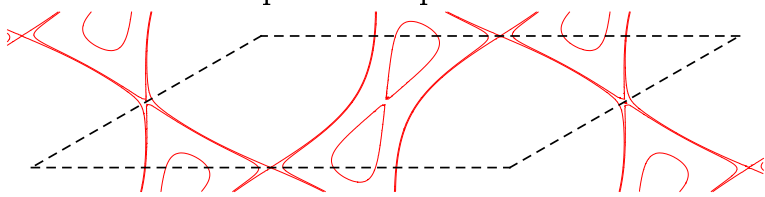

Separatrizes: ponto 256

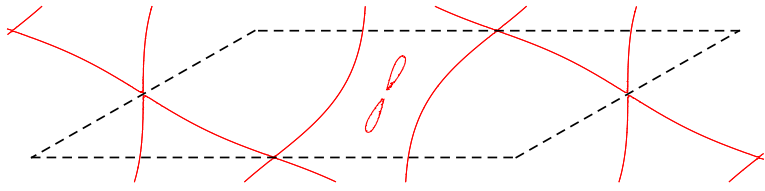

Separatrizes: ponto 26

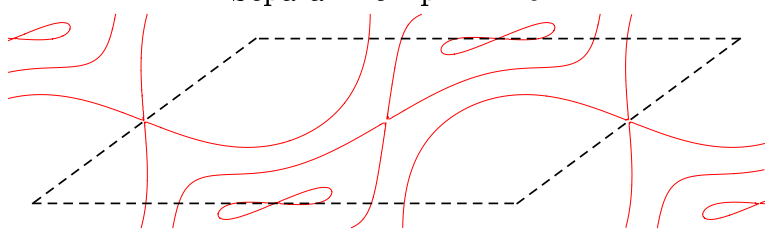

Separatrizes: ponto 261

Figura 5.14: Retratos de fase dos pontos 223, 23, 234, 24, 245, 25, 256, 26 e 261. 


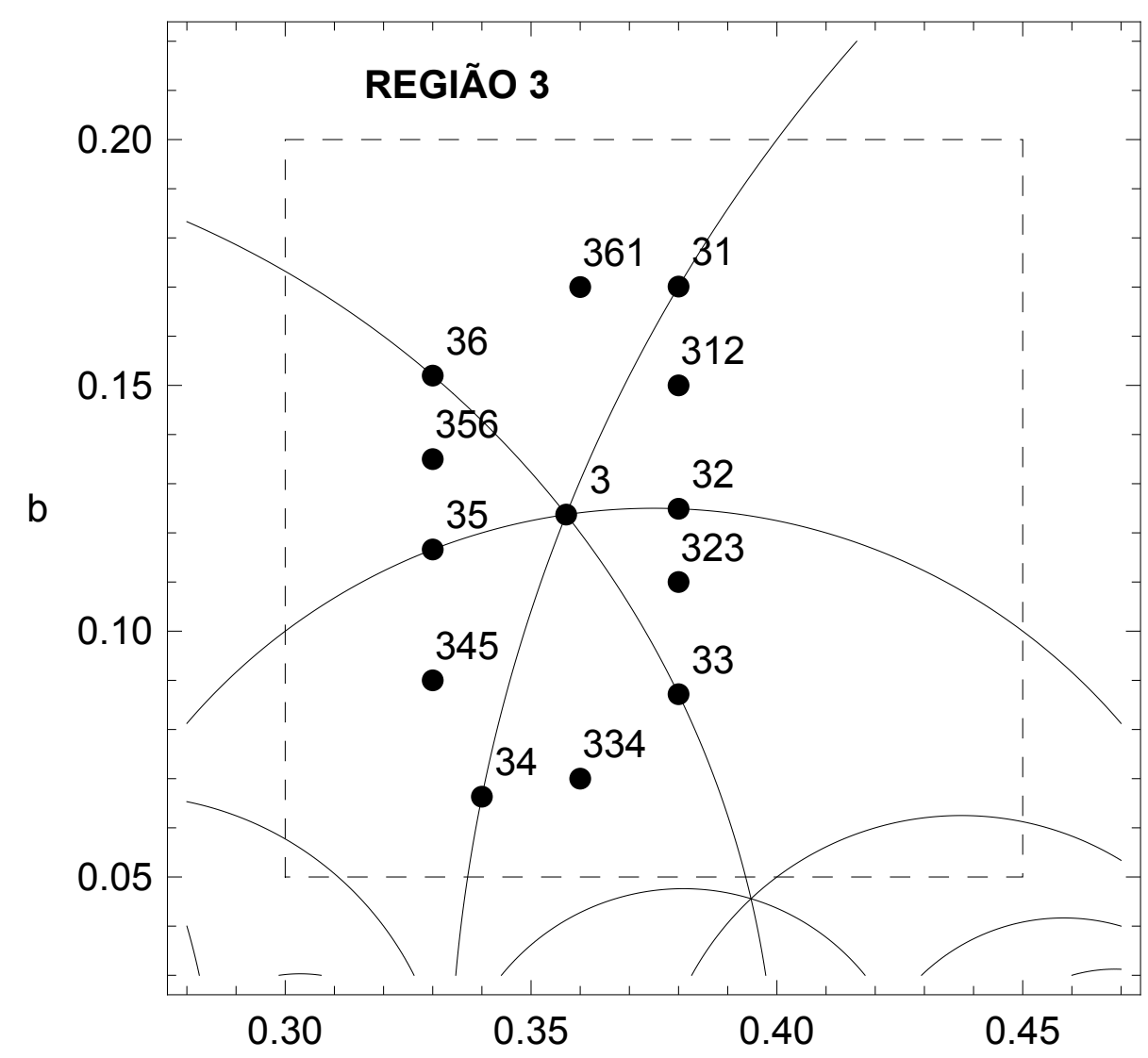

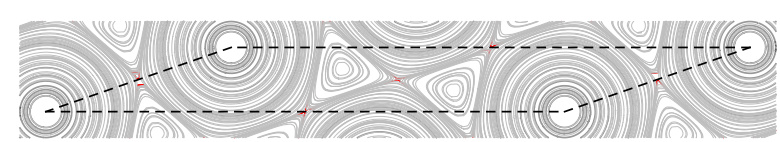

Curvas de nível: ponto 3

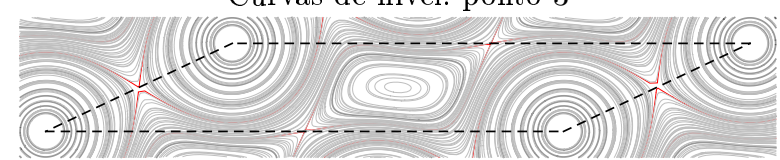

Curvas de nível: ponto 361

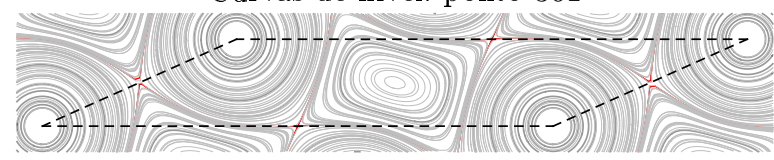

Curvas de nível: ponto 31

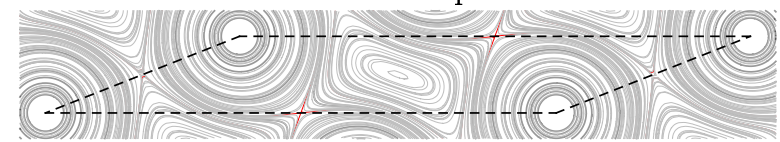

Curvas de nível: ponto 312

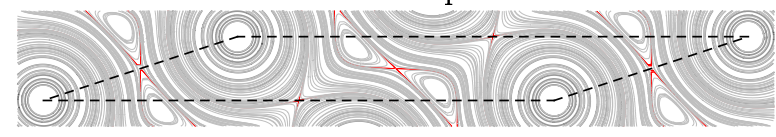

Curvas de nível: ponto 32

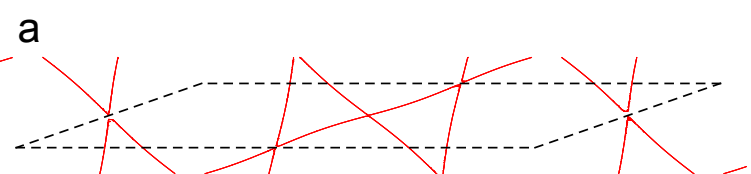

Separatrizes: ponto 3

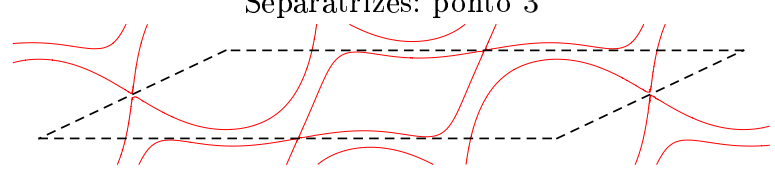

Separatrizes: ponto 361

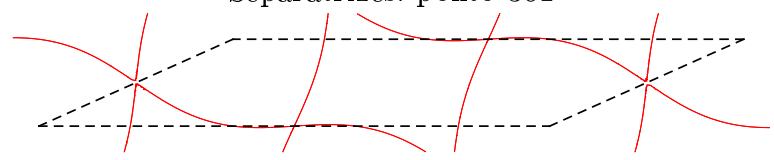

Separatrizes: ponto 31

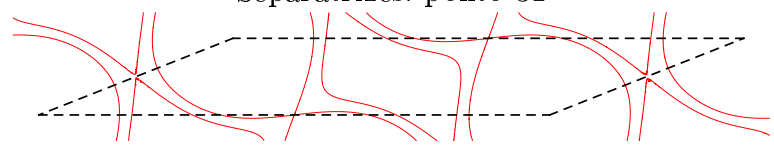

Separatrizes: ponto 312

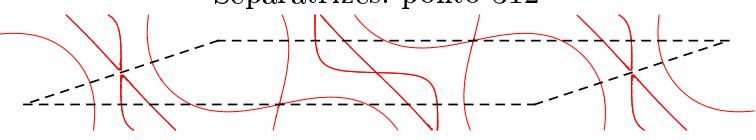

Separatrizes: ponto 32

Figura 5.15: Retratos de fase dos pontos 3,361, 31, 312 e 32 . 


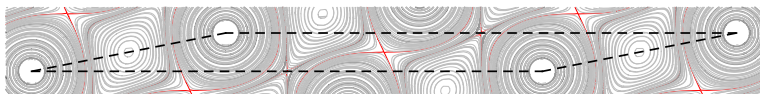

Curvas de nível: ponto 323

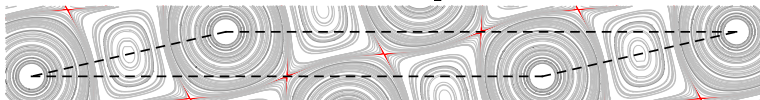

Curvas de nível: ponto 33

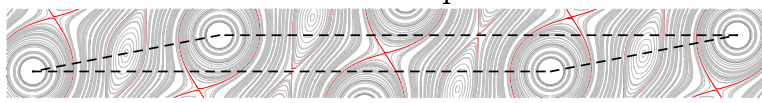

Curvas de nível: ponto 334

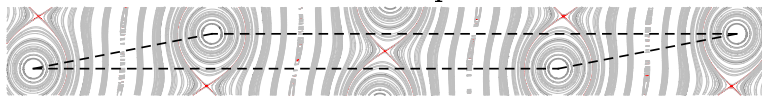

Curvas de nível: ponto 34

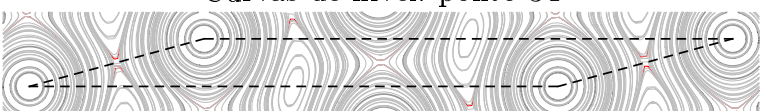

Curvas de nível: ponto 345

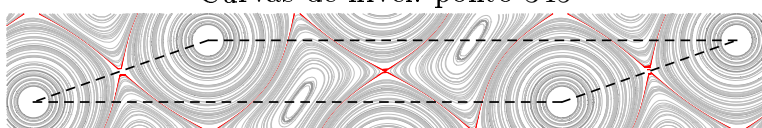

Curvas de nível: ponto 35

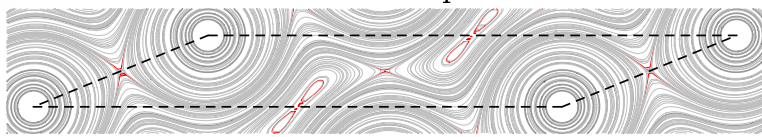

Curvas de nível: ponto 356

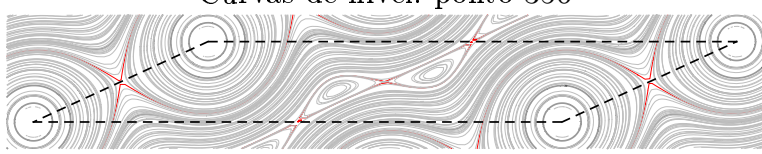

Curvas de nível: ponto 36

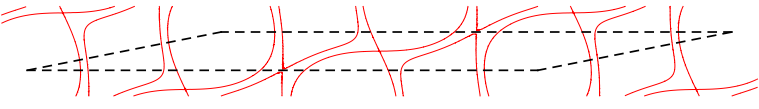

Separatrizes: ponto 323

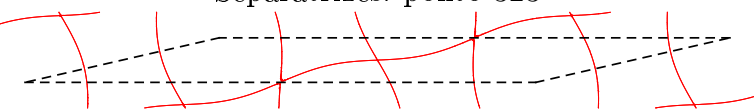

Separatrizes: ponto 33

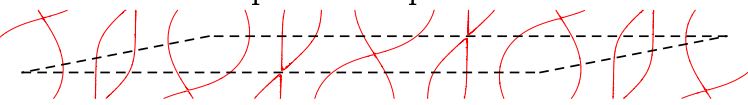

Separatrizes: ponto 334

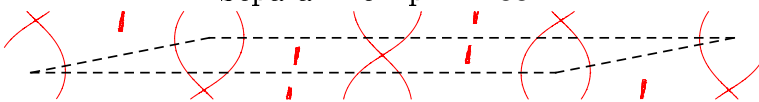

Separatrizes: ponto 34

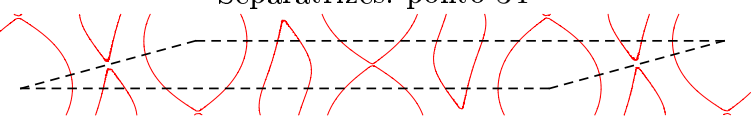

Separatrizes: ponto 345

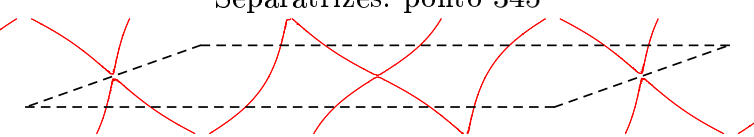

Separatrizes: ponto 35

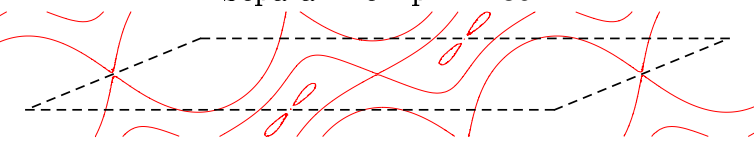

Separatrizes: ponto 356

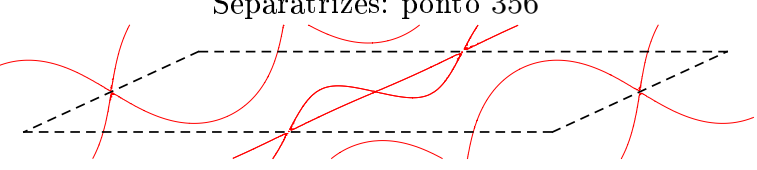

Separatrizes: ponto 36

Figura 5.16: Retratos de fase dos pontos $323,33,334,34,345,35,356$ e 36 . 


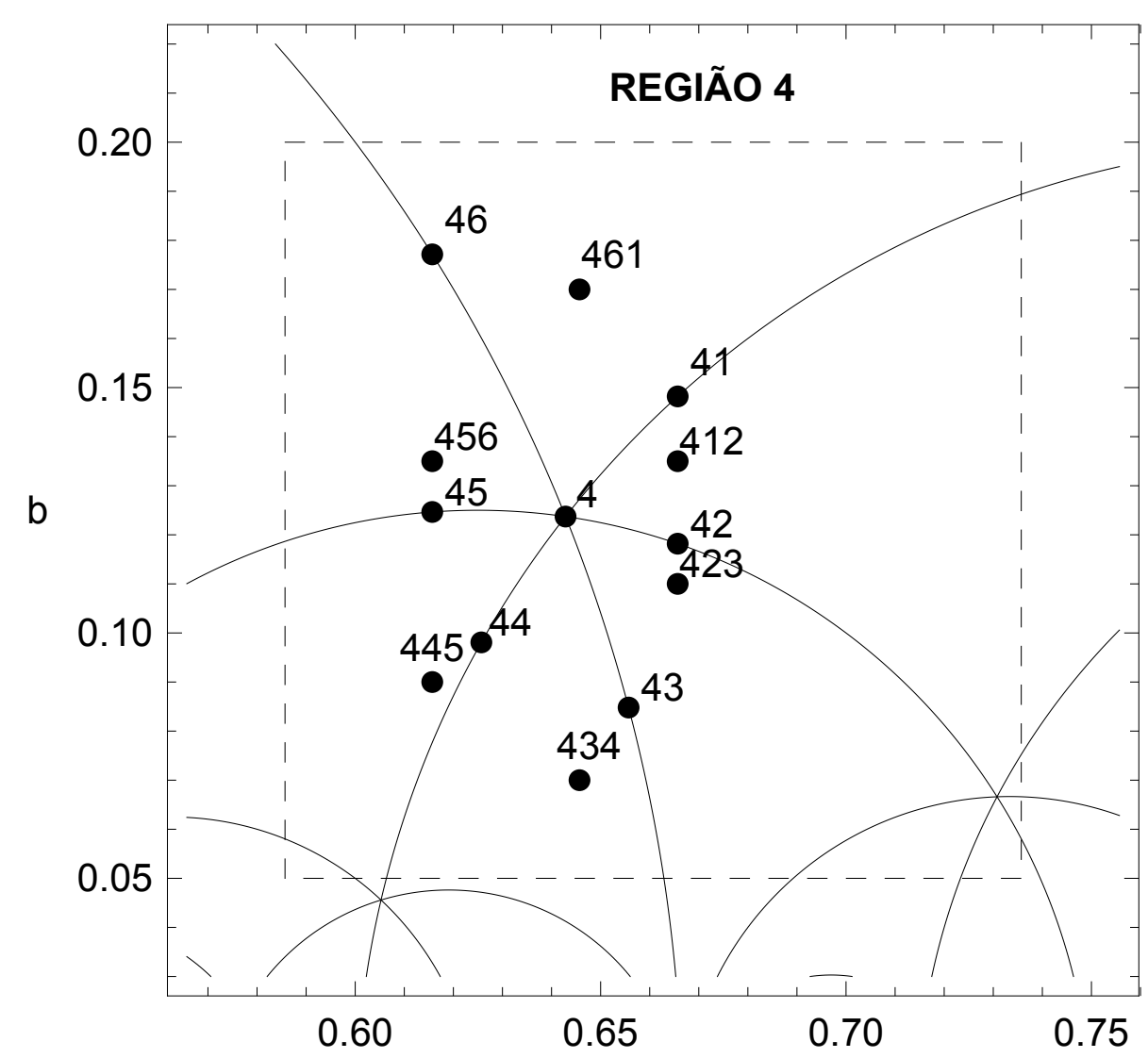

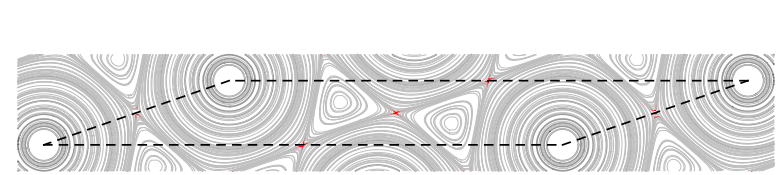

Curvas de nível: ponto 4

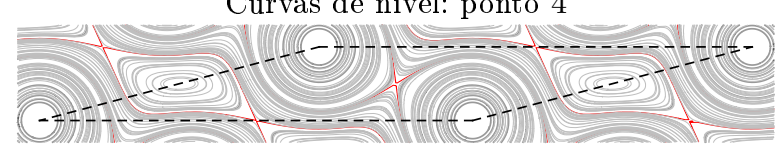

Curvas de nível: ponto 461

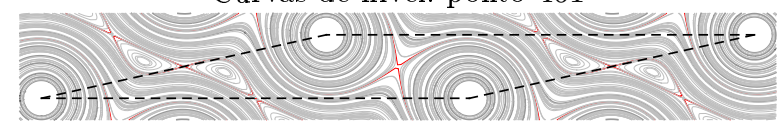

Curvas de nível: ponto 41

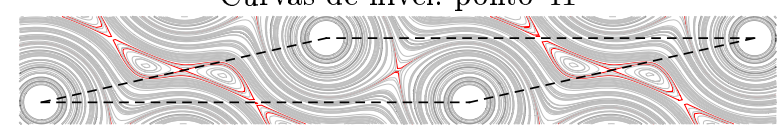

Curvas de nível: ponto 412

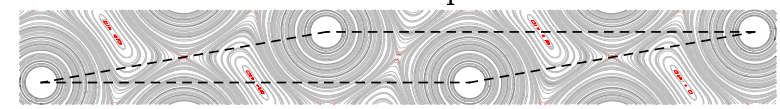

Separatrizes: ponto 42 a

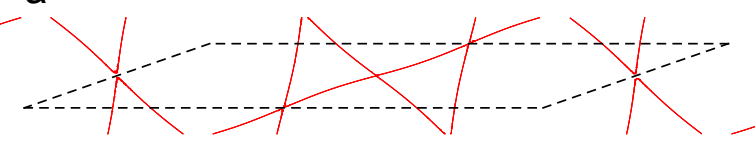

Separatrizes: ponto 4

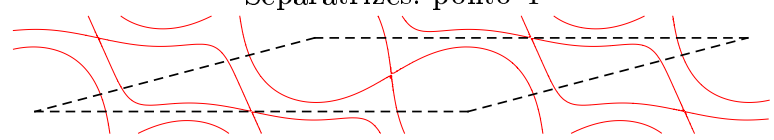

Separatrizes: ponto 461

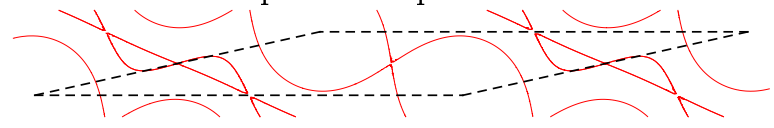

Separatrizes: ponto 41

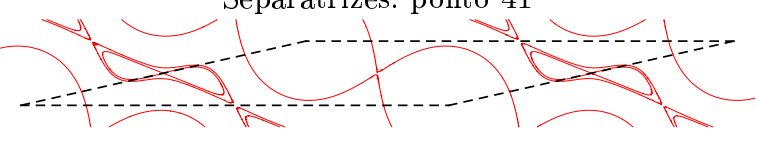

Separatrizes: ponto 412

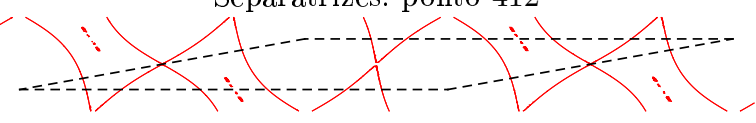

Separatrizes: ponto 42

Figura 5.17: Retratos de fase dos pontos 4, 461, 41, 412 e 42 . 


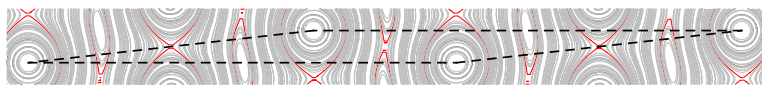

Curvas de nível: ponto 423

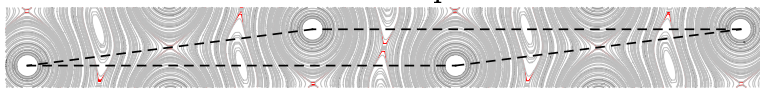

Curvas de nível: ponto 43

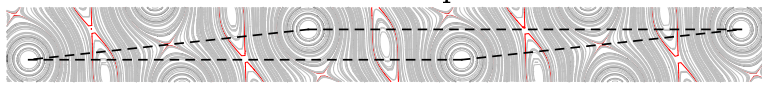

Curvas de nível: ponto 434

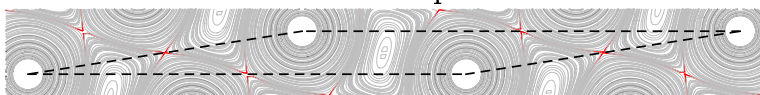

Curvas de nível: ponto 44

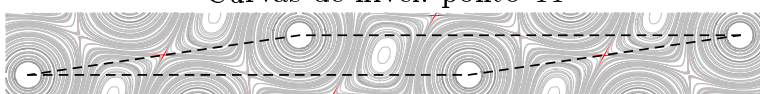

Curvas de nível: ponto 445

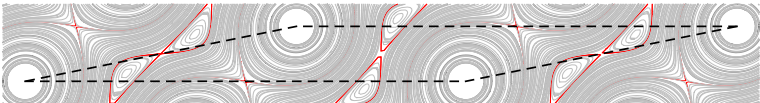

Curvas de nível: ponto 45

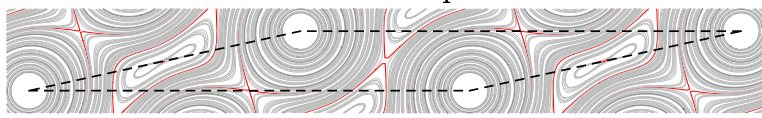

Curvas de nível: ponto 456

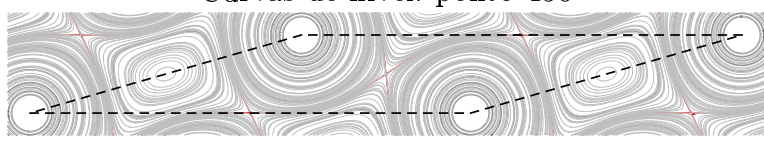

Curvas de nível: ponto 46

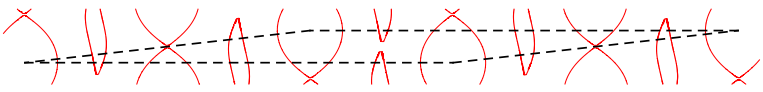

Separatrizes: ponto 423

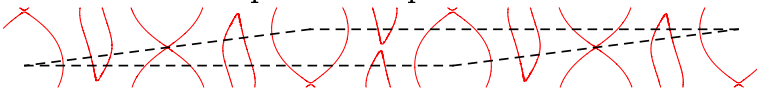

Separatrizes: ponto 43

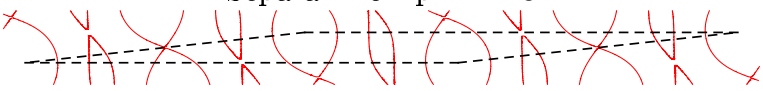

Separatrizes: ponto 434

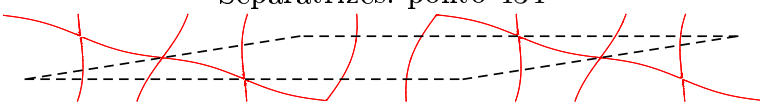

Separatrizes: ponto 44

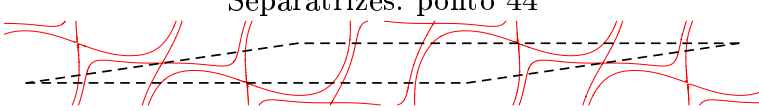

Separatrizes: ponto 445

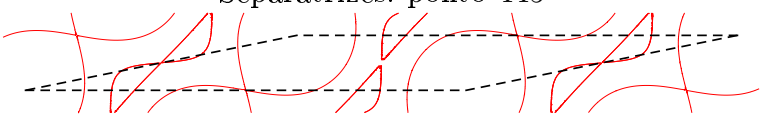

Separatrizes: ponto 45

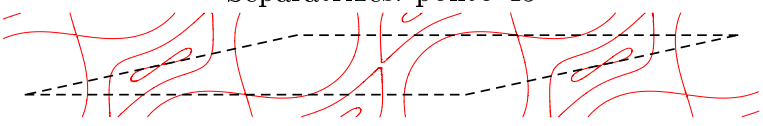

Separatrizes: ponto 456

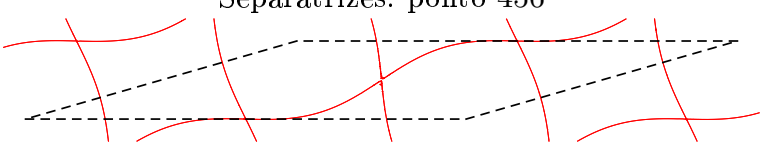

Separatrizes: ponto 46

Figura 5.18: Retratos de fase dos pontos $423,43,434,44,445,45,456$ e 46 . 


\subsubsection{Ação do grupo modular na estrutura das curvas de nível da função de Green do toro plano}

Nesta seção explicamos a estrutura dos diagramas de bifurcaçâo obtidos em termos da ação do grupo modular. Como ponto de partida, consideremos o reticulado $\Lambda=\left\{m g_{1}+n g_{2} \mid m, n \in \mathbb{Z}\right\}$ definido pelos geradores $g_{1}, g_{2} \in \mathbb{C}$ satisfazendo $g_{2} / g_{1} \in \mathbb{C} \backslash \mathbb{R}$, sendo $T=\mathbb{C} / \Lambda$ o toro correspondente. Definindo o domínio fundamental para representação do toro como o paralelogramo $\mathcal{P}$ com vértice na origem e lados dados por $0 g_{1}$ e $0 g_{2}$, o plano fica ladrilhado por cópias justapostas deste paralelogramo, conforme ilustrado na figura 5.19.

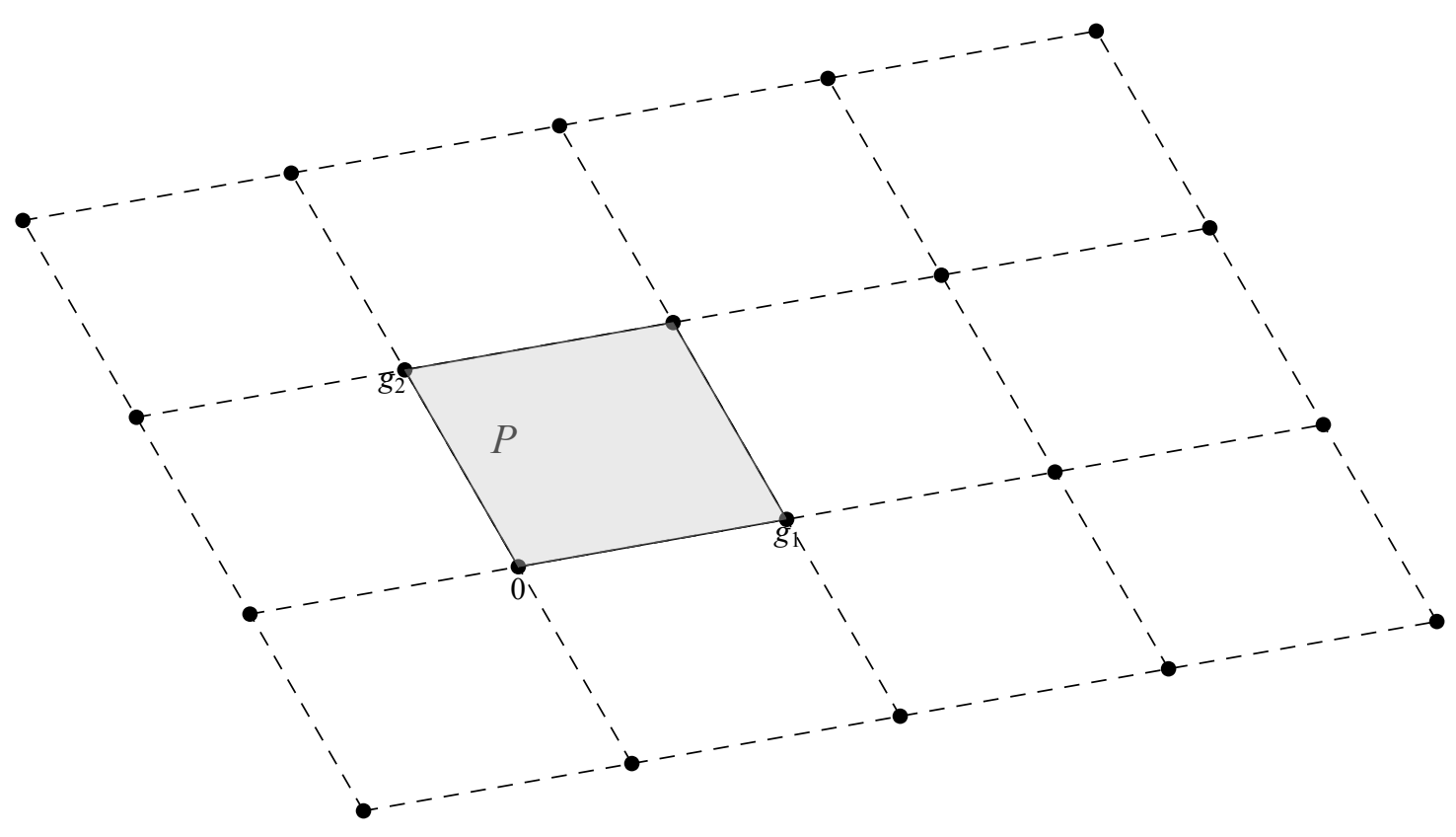

Figura 5.19: Reticulado no plano

Note que qualquer elemento $g \in \Lambda$ escreve-se de modo único na forma $g=n_{1} g_{1}+n_{2} g_{2}$ e, portanto, dizemos que o par $B=\left(g_{1}, g_{2}\right)$ define uma base de $\Lambda$. Dada outra base $\tilde{B}=\left(\tilde{g}_{1}, \tilde{g}_{2}\right)$ de $\Lambda$, fato conhecido é que a relação entre as duas bases é dada pela expressão ${ }^{3}$

$$
\begin{aligned}
& \tilde{g}_{1}=m_{11} g_{1}+m_{12} g_{2} \\
& \tilde{g}_{2}=m_{21} g_{1}+m_{22} g_{2}
\end{aligned},
$$

ou na forma matricial

$$
\left(\begin{array}{c}
\tilde{g}_{1} \\
\tilde{g}_{2}
\end{array}\right)=M\left(\begin{array}{l}
g_{1} \\
g_{2}
\end{array}\right)
$$

onde $M=\left(\begin{array}{cc}m_{11} & m_{12} \\ m_{21} & m_{22}\end{array}\right)$ com $m_{i j} \in \mathbb{Z}$ para $i, j \in\{1,2\}$. Além disso, como esta expressão pode ser invertida e seu determinante é um número inteiro, conclui-se que det $M=m_{11} m_{22}-m_{12} m_{21}= \pm 1$. Transformações do tipo 5.9, cujos coeficientes são inteiros e com determinante igual a \pm 1 , são denominadas transformaçôes unimodulares. Segue-se, portanto, que duas bases do mesmo reticulado estão relacionadas entre si por uma tranformação unimodular, conforme ilustra a figura 5.20. Isto implica na seguinte relação entre os parâmetros modulares:

$$
\tilde{\tau}=\frac{m_{22} \tau+m_{21}}{m_{12} \tau+m_{11}}
$$

Dentre todas as possíveis bases para um reticulado existe uma canônica, fato demonstrado no seguinte teorema

\footnotetext{
${ }^{3}$ Veja [Ahl79] páginas $266-270$.
} 


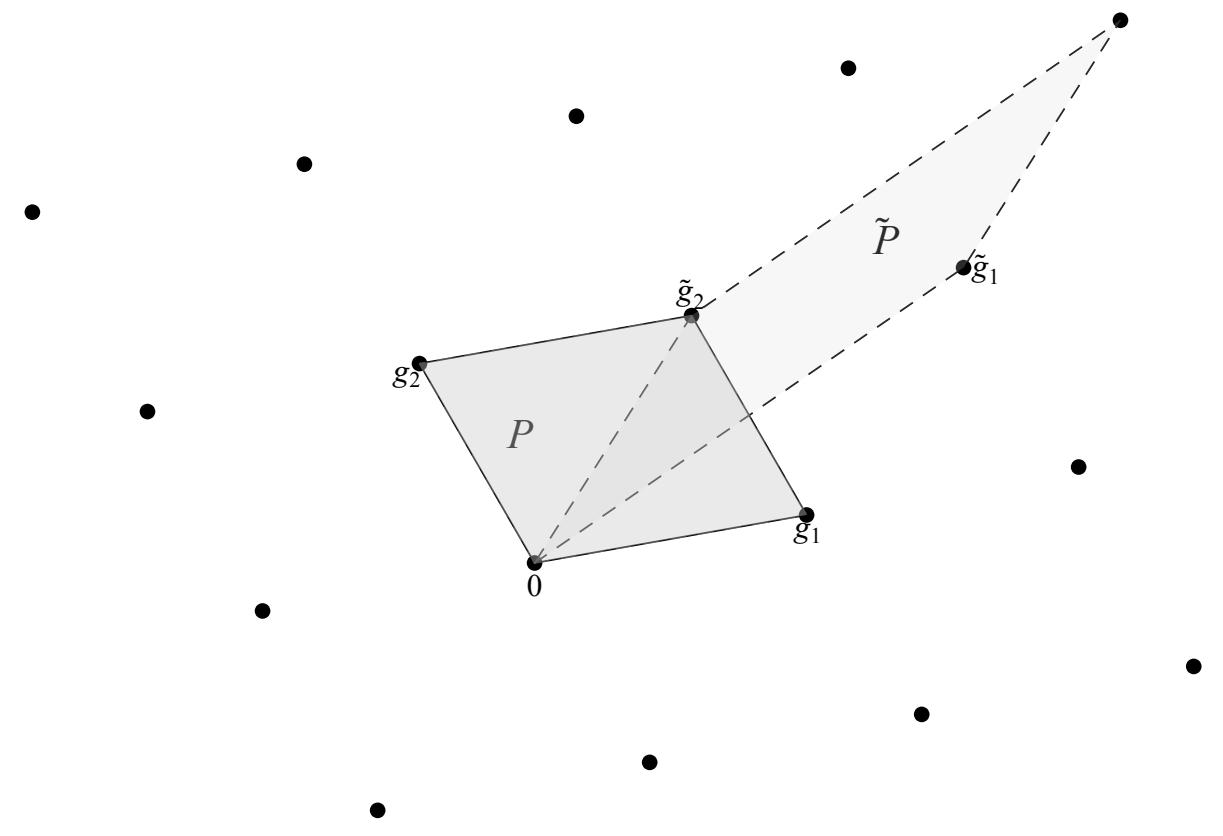

Figura 5.20: Ilustração de duas bases distintas para um mesmo reticulado, apresentando os dominios fundamentais correspondentes.

Teorema 4 (Ahlfors, pg. 268[Ahl'79]) Existe uma base $\left(g_{1}, g_{2}\right)$ tal que a razão $\tau=w_{2} / w_{1}$ satisfaz as seguintes condições: $(i) \operatorname{Im}(\tau)>0, \quad(i i)-\frac{1}{2}<\operatorname{Re}(\tau) \leq \frac{1}{2}, \quad(i i i)|\tau| \geq 1, \quad(i v) \operatorname{Re}(\tau) \geq 0$ se $|\tau|=1$. A razão $\tau$ é univocamente determinada por estas condiçôes, correspondendo a duas, quatro ou seis bases.

Note que se $\left(g_{1}, g_{2}\right)$ é uma base correspondente a $\tau,\left(-g_{1},-g_{2}\right)$ também o é. Para $\tau=i$ temos em correspondência 4 bases distintas, uma vez que $i$ é ponto fixo de $-1 / \tau$; para $\tau=e^{\pi i / 3}$ existem 6 bases distintas, considerando que $e^{\pi i / 3}$ é ponto fixo das transformações unimodulares $-(\tau+1) / \tau$ e $-1 /(1+\tau)$. O teorema 4 define um domínio fundamental $D$ para o parâmetro modular $\tau$ que gera um reticulado, conforme ilustrado na figura 5.21 ; isto é, dado um reticulado com parâmetro modular $\tilde{\tau} \in \mathbb{C} \backslash \mathbb{R}$, existe uma transformação unimodular que realiza uma mudança da base deste reticulado para uma nova base cujo parâmetro modular $\tau$ pertence a $D$.

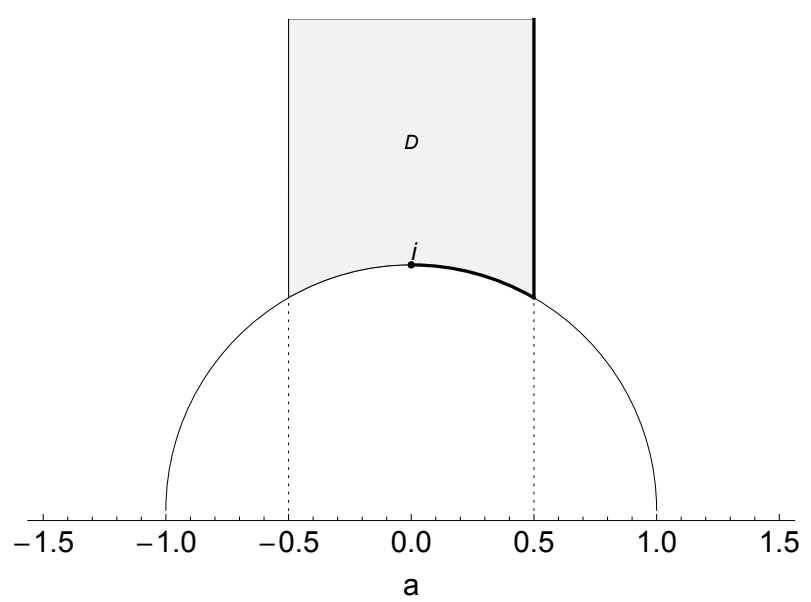

Figura 5.21: Domínio fundamental para o parâmetro modular $\tau$

Vejamos agora como estabelecer uma condição que caracteriza dois toros $T(\tau)$ e $T\left(\tau^{\prime}\right)$ biholomorficamente equivalentes, ou seja, tais que existe um biholomorfismo $f: T(\tau) \rightarrow T\left(\tau^{\prime}\right)$. 


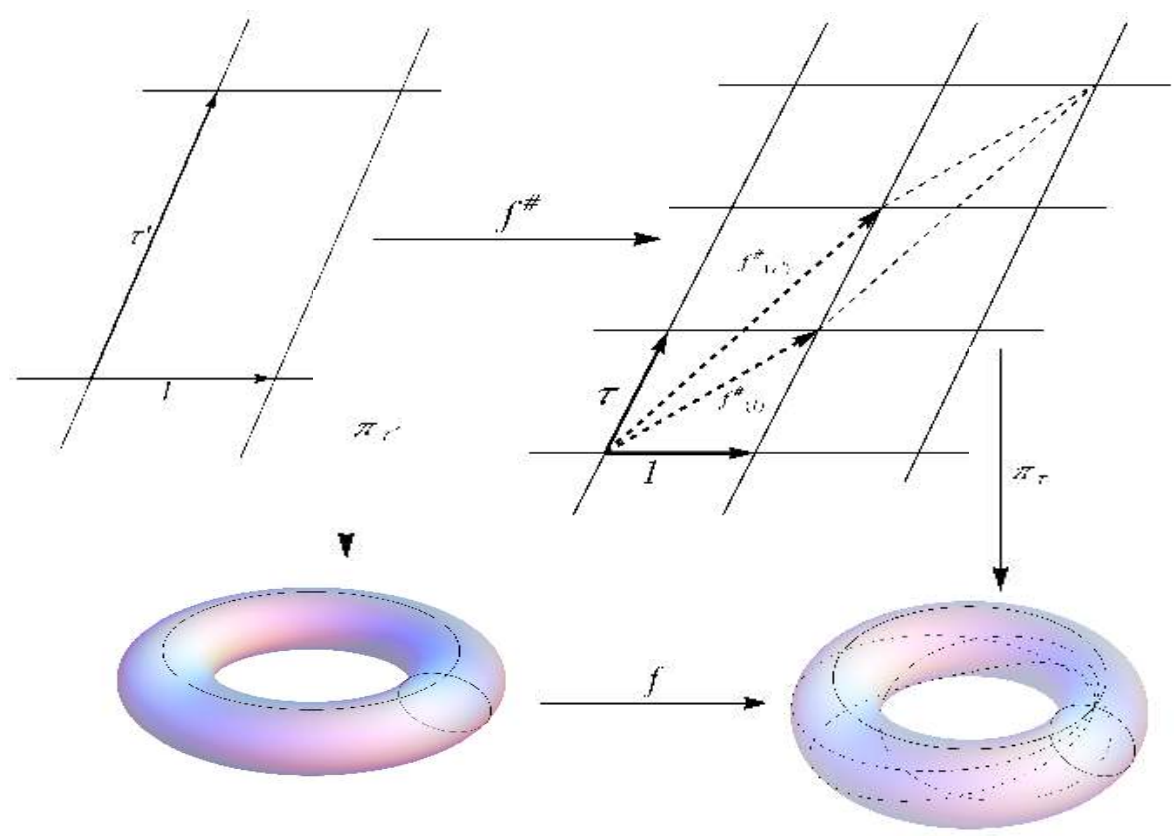

Figura 5.22: Toros biholomorficamente equivalentes

Teorema 5 (Imayoshi/Taniguchi, pg. 9[IT92]) Para quaisquer dois pontos $\tau$ e $\tau^{\prime}$ no semi-plano superior $\mathbb{H}$, os toros $T(\tau)$ e $T\left(\tau^{\prime}\right)$ são biholomorficamente equivalentes se e somente se $\tau$ e $\tau^{\prime}$ satisfazem a relação

$$
\tau^{\prime}=\frac{a \tau+b}{c \tau+d}
$$

onde $a, b, c$ e d são inteiros e $a d-b c=1$.

Conforme argumentado na demonstração deste teorema, um tal biholomorfismo $f$ admite um levantamento $f^{\#}: \mathbb{C} \rightarrow \mathbb{C}$ que é um biholomorfismo do plano complexo satisfazendo $\pi_{\tau} \circ f^{\#}=f \circ \pi_{\tau^{\prime}}$; exigindo que $f^{\#}$ fixe a origem, temos $f^{\#}(z)=\alpha z$ com $\alpha \neq 0$. A demonstração do teorema segue destes fatos. Note que, conforme visto anteriormente, os coeficiente inteiros $a, b, c$ e $d$ no teorema acima definem uma transformação unimodular que realiza uma mudança de base no reticulado $\Lambda(\tau)$, conforme ilustrado na figura 5.22. Desta forma, existe uma equivalência entre classes de equivalências de toros planos e reticulados do plano: toros biholomorficamente equivalentes correspondem a domínios fundamentais definidos por diferentes bases em um mesmo reticulado do plano. O conjunto dos automorfismos biholomorfos do semi-plano superior $\mathbb{H}$ define o grupo modular, conforme expressão abaixo

$$
P S L(2, \mathbb{Z}):=\left\{g_{\tau}=\frac{a \tau+b}{c \tau+d} \mid a, b, c, d \in \mathbb{Z} \text { e } a d-b c=1\right\} .
$$

Pelo teorema 5, concluímos que o espaço de moduli de toros, denotado por $M_{1}$ e definido como sendo o conjunto de todas as classes de equivalências de toros por biholomorfismos, ou seja,

$$
M_{1} \cong \mathbb{H} / \operatorname{PSL}(2, \mathbb{Z})
$$

pode ser representado pelo domínio fundamental $D$ definido no teorema 4 . Note que o grupo modular é o subgrupo do grupo unimodular composto pelos elementos cujo determinante é igual a 1 e portanto, como estamos restringindo a ação ao semi-plano superior, a caracterização do domínio fundamental para o grupo modular acima apresentada é imediata. De fato, considerando que a 
relação entre $\tau^{\prime}$ e $\tau$ pela ação do grupo unimodular é

$$
\tau^{\prime}=\frac{m_{22} \tau+m_{21}}{m_{12} \tau+m_{11}}
$$

verifica-se facilmente que

$$
\Im\left(\tau^{\prime}\right)=\frac{m_{11} m_{22}-m_{21} m_{12}}{\left|m_{11}+m_{12} \tau\right|^{2}} \Im(\tau)=\frac{\operatorname{det}\left(\begin{array}{cc}
m_{11} & m_{12} \\
m_{21} & m_{22}
\end{array}\right)}{\left|m_{11}+m_{12} \tau\right|^{2}} \Im(\tau),
$$

onde $\operatorname{det}\left(\begin{array}{cc}m_{11} & m_{12} \\ m_{21} & m_{22}\end{array}\right)= \pm 1$, de modo que o semi-plano superior é invariante pela ação do grupo modular. Veremos agora que a escolha de um parâmetro modular $\tau \in \mathbb{H}$ corresponde à fixar uma base do grupo fundamental do toro, de modo que a ação do grupo modular em $\mathbb{H}$ corresponde à mudança de base do grupo fundamental. Conforme apresentado em [IT92], escolhendo o ponto base $p_{0}=[0]$ do grupo fundamental $\pi_{1}\left(T(\tau), p_{0}\right)$ de $T(\tau)$, os segmentos definidos pelos pontos 0 e 1 e por 0 e $\tau$ definem curvas fechadas simples $C(1)$ e $C(\tau)$ em $T(\tau)$ cujas classes de homotopia $[C(1)]$ e $[C(\tau)]$ fornecem geradores canônicos de $\pi_{1}\left(T(\tau), p_{0}\right)$, de modo que a correspondência entre $1, \tau$ e $[C(1)],[C(\tau)]$ define um isomorfismo entre $\Lambda(\tau)$ e $\pi_{1}\left(T(\tau), p_{0}\right)$. Portanto, uma transformação biholomorfa $f: T\left(\tau^{\prime}\right) \rightarrow T(\tau)$ induz um isomorfismo canônico $f_{*}: \pi_{1}\left(T\left(\tau^{\prime}\right), p_{0}\right) \rightarrow \pi_{1}\left(T(\tau), p_{0}\right)$ definido da maneira usual. Define-se uma marcação em um toro $T$ como sendo uma escolha de um sistema canônico de geradores do grupo fundamental $\pi_{1}(T, p)$ de $T$, representado por $\Sigma_{p}=$ $\{[C],[\tilde{C}]\}$. Duas marcações $\Sigma_{p_{1}}=\left\{\left[C_{1}\right],\left[\tilde{C}_{1}\right]\right\}$ e $\Sigma_{p_{2}}=\left\{\left[C_{2}\right],\left[\tilde{C}_{2}\right]\right\}$ são ditas equivalentes quando existe uma curva contínua $l_{1,2}$ de $p_{1}$ a $p_{2}$ que induz um isomorfismo $I_{1,2}$ de $\pi_{1}\left(T, p_{1}\right)$ em $\pi_{1}\left(T, p_{2}\right)$ tal que $\left[C_{2}\right]=I_{1,2}\left(\left[C_{1}\right]\right)$ e $\left[\tilde{C}_{2}\right]=I_{1,2}\left(\left[\tilde{C}_{1}\right]\right)$. Desta forma, dizemos que dois pares $\left(T_{1}, \Sigma_{p}\right)$ e $\left(T_{2}, \Sigma_{q}\right)$ são equivalentes se e somente se existe uma aplicação biholomorfa $f: T_{1} \rightarrow T_{2}$ tal que $f_{*}\left(\Sigma_{p}\right)$ é equivalente a $\Sigma_{q}$. Denominamos por toro marcado à classe de equivalência $\left[T, \Sigma_{p}\right]$ do par $\left(T, \Sigma_{p}\right)$. O espaço de Teichmüller $T_{1}$ de gênero 1 consiste no conjunto de todos os toros marcados. O seguinte teorema estabelece uma correspondência entre $T_{1}$ e o semi-plano superior $\mathbb{H}$.

Teorema 6 (Imayoshi/Taniguchi,pg. 12[IT92]) Para todo ponto $\tau \in \mathbb{H}, \operatorname{seja} \Sigma(\tau)=\{[C(1), C(\tau)]\}$ uma marcação em $T(\tau)=\mathbb{C} \backslash \Lambda(\tau)$ para a qual $[C(1)]$ e $[C(\tau)]$ correpondem a 1 e $\tau$ em $\Lambda(\tau)$, respectivamente. Então $[T(\tau), \Sigma(\tau)]=\left[T\left(\tau^{\prime}\right), \Sigma\left(\tau^{\prime}\right)\right]$ em $T_{1}$ se e somente se $\tau=\tau^{\prime}$.

Agora veremos como os fatos acima mencionados nos auxiliam na compreensão dos diagramas de bifurcação apresentados no capítulo anterior. Vimos que um toro plano $T$ é representado como o quociente de $\mathbb{C}$ por um reticulado normalizado $\Lambda(\tau)$, onde escolhemos como domínio fundamental do toro o paralelogramo $\mathcal{P}$ definido pela origem e pelos pontos 1 e $\tau$. A função de Green $G$ para o laplaciano em $T$ admite um levantamento para o plano complexo que, por abuso de notação, também denotamos por $G(z ; \tau)$. Note que esta função possui singularidades logarítmicas nos pontos do reticulado (vórtices) e satisfaz

$$
\Delta G(z)=-\delta_{[0]}(z)+\frac{1}{|T|},
$$

onde [0] representa os pontos de $\mathbb{C}$ que estão na mesma classe da origem com respeito à ação do grupo $\Lambda(\tau)$, isto é, os vértices do reticulado, e $|T|$ é a área do toro. Além disso, vale que

$$
\iint_{\mathcal{P}} G(z) d \mu=0
$$

Desta forma, dada uma base $\left(g_{1}^{\prime}, g_{2}^{\prime}\right)$ do mesmo reticulado $\Lambda(\tau)$ mas diferente da canônica $(1, \tau)$, o paralelogramo fundamental $\mathcal{P}^{\prime}$ definido pela origem e por $g_{1}^{\prime}$ e $g_{2}^{\prime}$ define um toro $T^{\prime}$ que está na mesma classe conforme de $T$. Além disso, como o determinante da transformação de mudança de 
base é igual a 1 temos que $\left|T^{\prime}\right|=|T|$ e portanto verifica-se que

$$
\Delta G(z)=-\delta_{[0]}(z)+\frac{1}{\left|T^{\prime}\right|}
$$

e

$$
\iint_{\mathcal{P}^{\prime}} G(z) d \mu=0
$$

de modo que os levantamentos das funções de Green em $T$ e $T^{\prime}$ para $\mathbb{C}$ são representados pela mesma função $G$. Como o toro $T^{\prime}$ definido por $\left(g_{1}^{\prime}, g_{2}^{\prime}\right)$ não está em sua forma normalizada, determinemos o "pull-back" da função de Green em $T^{\prime}$ pela transformação conforme $z=f(p)=g_{1}^{\prime} p$ que leva os geradores canônicos $\left(1, \tau^{\prime}\right)$ em $\left(g_{1}^{\prime}, g_{2}^{\prime}\right)$, onde $\tau^{\prime}=g_{2}^{\prime} / g_{1}^{\prime}, g_{1}^{\prime}=2 w_{1}$ e $g_{2}^{\prime}=2 w_{2}$. Para tanto, faz-se necessário apresentar a expressão geral da função de Green para o toro plano quando este é representado pelo quociente de $\mathbb{C}$ pelo reticulado gerado por $\Lambda=2 m w_{1}^{\prime}+2 n w_{2}^{\prime}$ onde $m, n \in \mathbb{Z}$ e $w_{1}^{\prime}$ e $w_{2}^{\prime}$ são os meio-períodos representados por dois números complexos quaisquer satisfazendo $\tau=w_{2}^{\prime} / w_{1}^{\prime} \notin \mathbb{R}$. Nesta situação, temos

$$
G\left(z ; w_{1}^{\prime}, w_{2}^{\prime}\right)=-\frac{1}{2 \pi}\left[\ln \left|2 w_{1}^{\prime} \frac{\vartheta_{1}\left(z / 2 w_{1}^{\prime} ; \tau^{\prime}\right)}{\vartheta_{1}^{\prime}\left(\mathbf{0} ; \tau^{\prime}\right)}\right|+\frac{\pi}{\Delta} \frac{\left(w_{1}^{\prime} \bar{z}-z \bar{w}_{1}^{\prime}\right)^{2}}{4\left|w_{1}^{\prime}\right|^{2}}\right],
$$

onde $\Delta=2 i\left(w_{1}^{\prime} \bar{w}_{2}^{\prime}-\bar{w}_{1}^{\prime} w_{2}^{\prime}\right)=2 i\left|w_{1}^{\prime}\right|^{2}\left(\bar{\tau}^{\prime}-\tau^{\prime}\right)$. Portanto, segue-se o desenvolvimento

$$
\begin{aligned}
f^{*} G\left(p ; \tau^{\prime}\right) & =-\frac{1}{2 \pi}\left[\ln \left|2 w_{1}^{\prime} \frac{\vartheta_{1}\left(p ; \tau^{\prime}\right)}{\vartheta_{1}^{\prime}\left(\mathbf{0} ; \tau^{\prime}\right)}\right|+\frac{\pi}{2 i\left|w_{1}^{\prime}\right|^{2}\left(\bar{\tau}^{\prime}-\tau^{\prime}\right)} \frac{\left(2\left|w_{1}^{\prime}\right|^{2} \bar{p}-2 p\left|w_{1}^{\prime}\right|^{2}\right)^{2}}{4\left|w_{1}^{\prime}\right|^{2}}\right] \\
& =-\frac{1}{2 \pi} \ln \left|2 w_{1}^{\prime}\right|+G\left(p ; 1 / 2, \tau^{\prime} / 2\right),
\end{aligned}
$$

isto é, a função de Green definida no reticulado normalizado difere do "pull-back" da função de Green no reticulado geral por uma constante, donde conclui-se que os campos hamiltonianos correspondentes são iguais e a transformação conforme $f$ estabelece uma conjugação entre o campo no reticulado geral e o campo no reticulado normalizado. Dos resultados acima apresentados conclui-se que, a menos da classe de homotopia das separatrizes, o retrato de fase para a dinâmica do centro de vorticidade de um sistema de dois vórtices no toro plano $T\left(\tau^{\prime}\right)$ fica completamente descrito pelo retrato de fase correspondente ao representante $\tau$ da classe de equivalência $\left[\tau^{\prime}\right]$ pela ação do grupo modular e que pertence ao domínio fundamental $\mathcal{D}$. Para ilustrar este fato, consideremos o retrato de fase do sistema correspondente ao ponto identificado pelo rótulo 234 na região 2 do diagrama de análise da bifurcação das separatrizes, que corresponde a $\tau_{0}=0.52+0.25 i$ e é representado na figura abaixo:

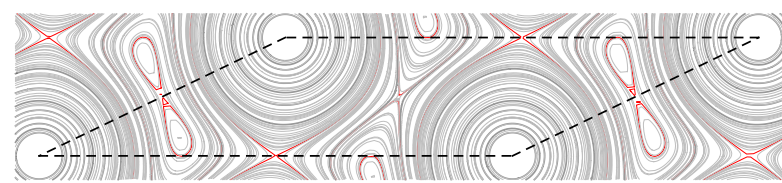

a) Curvas de nível: ponto 234

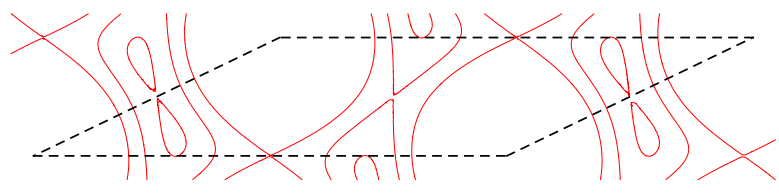

b) Separatrizes: ponto 234

Figura 5.23: a) retrato de fase do sistema correspondente ao ponto 234 e b) representação de suas conexões homoclinicas e heteroclinicas

$\mathrm{Na}$ primeira figura em 5.24 são representadas as separatrizes do sistema correspondente no reticulado $\Lambda\left(\tau_{0}\right)$. Obtemos uma sequência de três transformações modulares $M_{1}, M_{2}$ e $M_{3}$ tal que a resultante $M=M_{3} \circ M_{2} \circ M_{1}$ da composição das três satisfaz $M\left(\tau_{0}\right) \in D$. Neste exemplo encontramos que $M_{1}(\tau)=\tau-1$ e $M_{2}=M_{3}$ agem no parâmetro modular da seguinte forma:

$$
M_{2}(\tau)=\frac{\tau}{1+\tau}=M_{3}(\tau),
$$

sendo o resultado final ilustrado na figura 5.25 b). Na figura 5.28 vemos a representação destes parâmetros modulares no espaço de parâmetros, onde definimos $\tau_{1}:=M_{1}\left(\tau_{0}\right), \tau_{2}:=M_{2}\left(\tau_{1}\right)$ e 
$\tau_{3}:=M_{3}\left(\tau_{2}\right)$

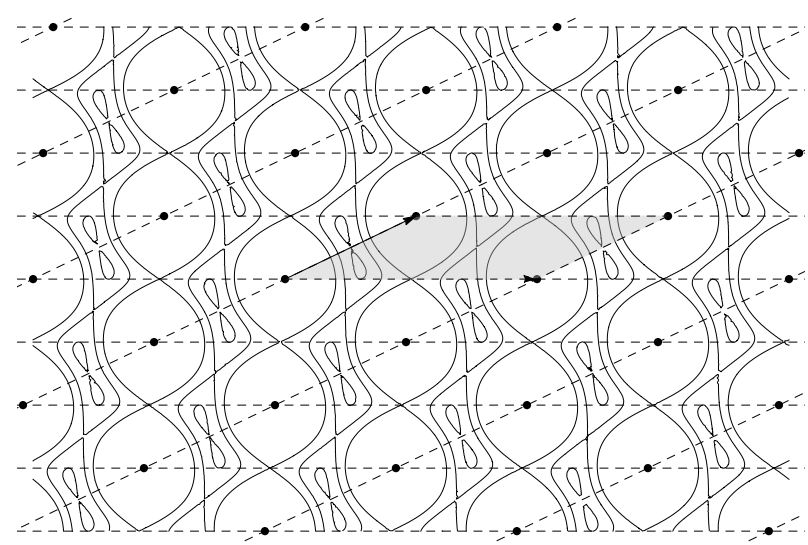

a) separatrizes de $G\left(z ; \tau_{0}\right)$ para $\tau_{0}=0.52+0.25 i$

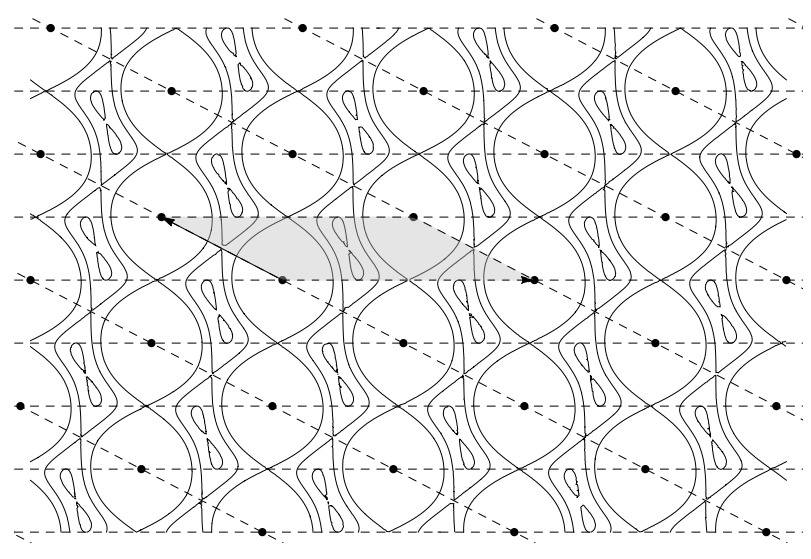

b) separatrizes de $G(z ; \tau)$, onde $\tau=M_{1}\left(\tau_{0}\right)$

Figura 5.24: Representação das separatrizes de $G(z ; \tau)$ e do reticulado $\Lambda(\tau)$ com geradores $g_{1}=1$ e $g_{2}=\tau$ onde: $a) \tau=0.52+0.25 i$ e b) $\tau=\tau_{0}-1=-0.48+0.25 i$

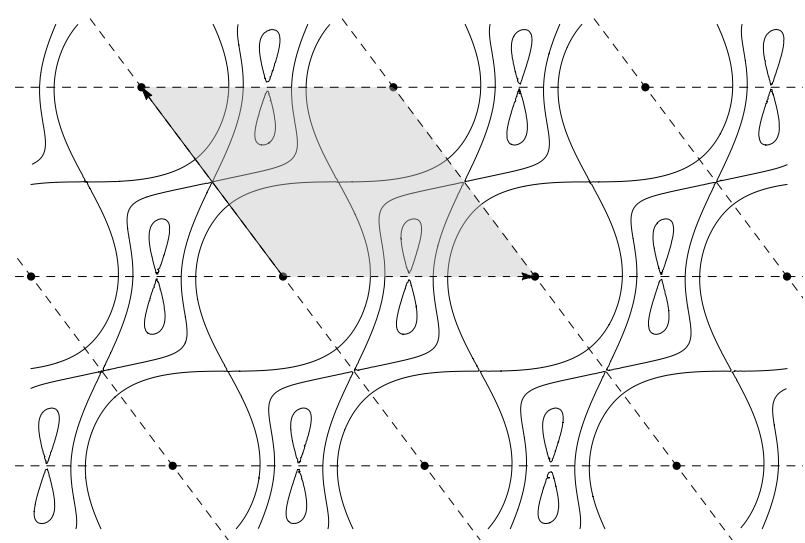

a) separatrizes de $G(z ; \tau)$ para $\tau=\tau_{2}$

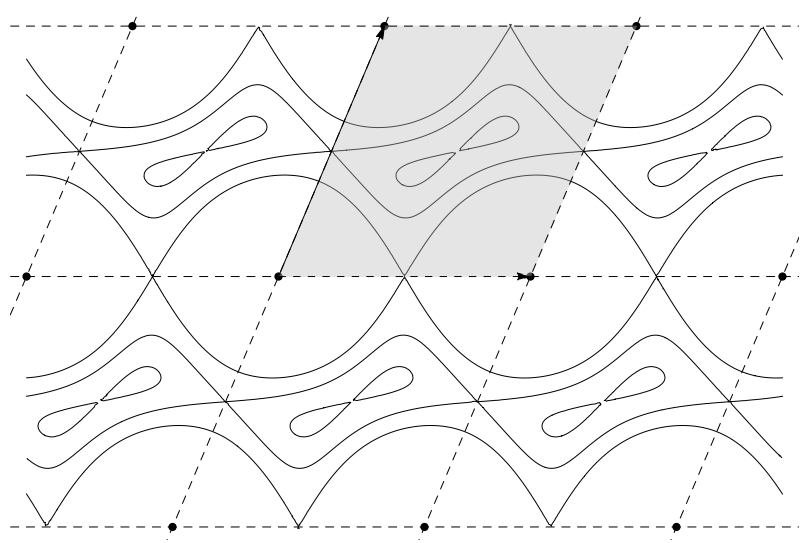

b) separatrizes de $G(z ; \tau)$, onde $\tau=\tau_{3}$

Figura 5.25: Representação das separatrizes de $G(z ; \tau)$ e do reticulado $\Lambda(\tau)$ com geradores $g_{1}=1$ e $g_{2}=\tau$ onde: $a) \tau=\tau_{2}$ e b) $\tau=\tau_{3}$

Vejamos agora o resultado que obtemos ao agir com estas mesmas transformações modulares mas efetuando mudanças de base no reticulado inicial $\Lambda\left(\tau_{0}\right)$. O resultado é ilustrado nas figuras 5.26 e 5.27 .

Observe que a relação entre os reticulados representados em 5.27 e 5.25 é estabelecida pela aplicação conforme $f(z)=z / g_{1}$ que normaliza a base do reticulado. Como mostrado anteriormente, os sistemas correspondentes por tal renormalização são conjugados. Na figura 5.28 os valores do parâmetro modular $\tau$ para cada situação acima é representado.

Na figura 5.29 observamos os diagramas de bifurcação no espaço de Moduli de toros, representado pelo domínio $\mathcal{D}$. A fim de descrever a mudança dos retratos de fase correspondentes a pontos próximos das curvas que delimitam este domínio, selecionamos os pontos apresentados na figura 5.30 e listamos os retratos de fase dos sistemas correspondentes nas figuras 5.30, 5.31 e 5.32 . 


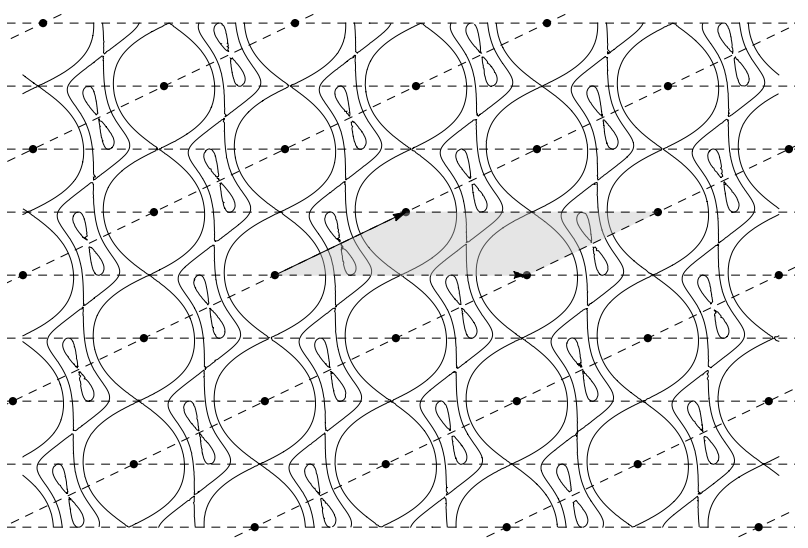

a)

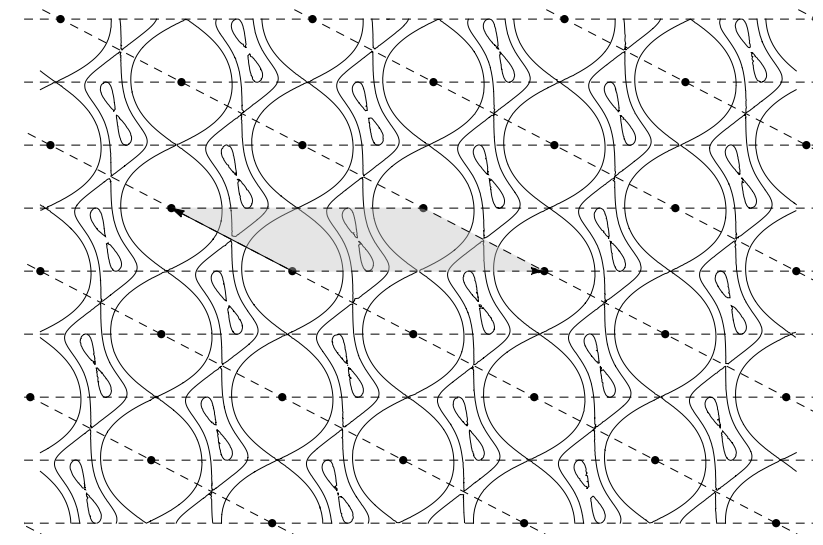

b)

Figura 5.26: a) representação das separatrizes de $G\left(z ; w_{1}, w_{2}\right)$ e do reticulado $\Lambda\left(g_{1}, g_{2}\right)$ gerado pela base inicial $g_{1}=1=2 w_{1}$ e $\left.g_{2}=\tau_{0}=2 w_{2} ; b\right)$ representação das separatrizes de $G\left(z ; w_{1}, w_{2}\right)$ e do reticulado $\Lambda\left(g_{1}, g_{2}\right)$ correspondentes à base $B_{1}$ obtida a partir da base inicial pela ação de $M_{1}$.

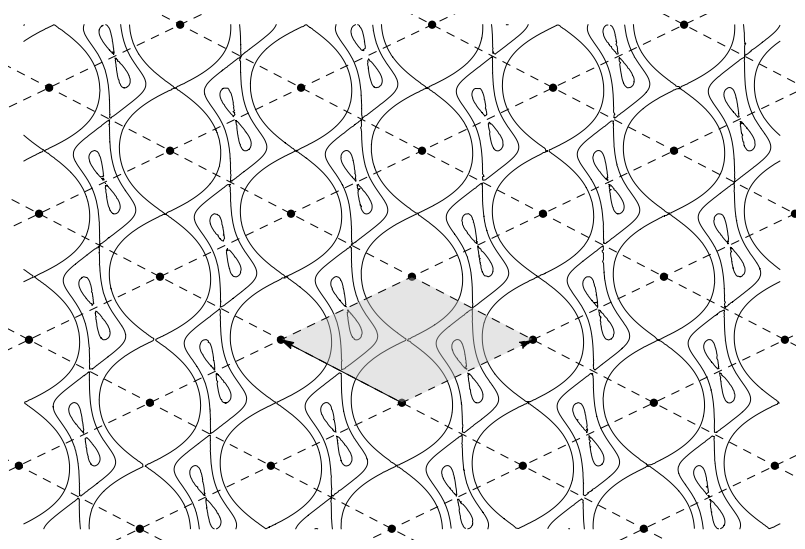

a)

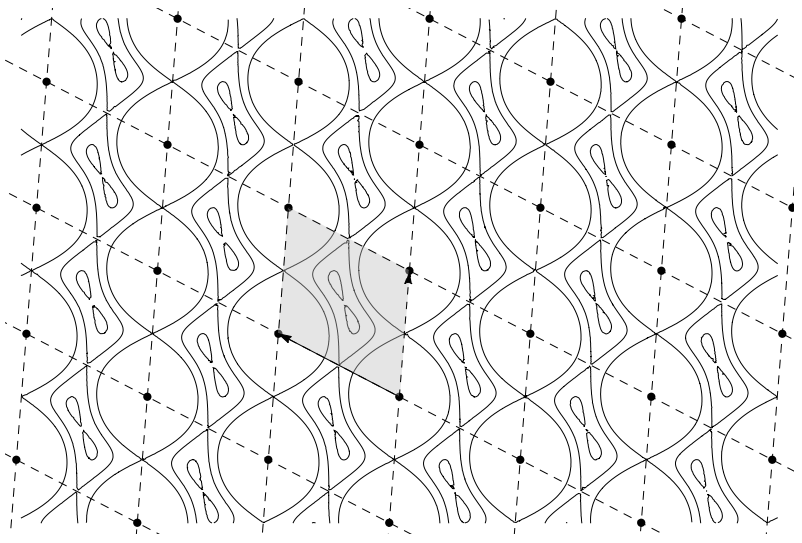

b)

Figura 5.27: a) representação das separatrizes de $G\left(z ; w_{1}, w_{2}\right)$ e do reticulado $\Lambda\left(g_{1}, g_{2}\right)$ correspondentes $\grave{a}$ base $B_{2}$ obtida a partir da base $B_{1}$ pela ação de $\left.M_{2} ; b\right)$ representação das separatrizes de $G\left(z ; w_{1}, w_{2}\right)$ e do reticulado $\Lambda\left(g_{1}, g_{2}\right)$ correspondentes à base $B_{3}$ obtida a partir da base $B_{2}$ pela ação de $M_{3}$. 


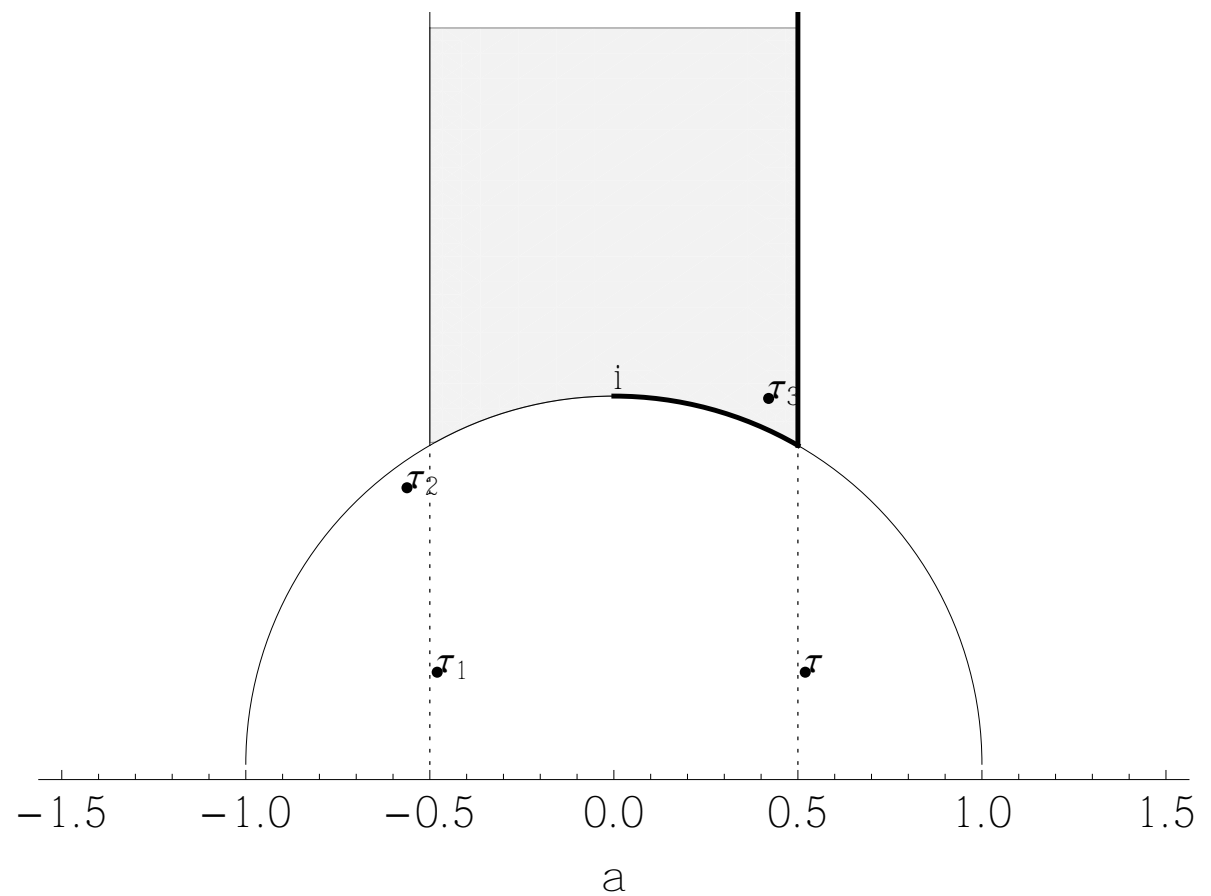

Figura 5.28: Imagens de $\tau=\tau_{0}$ pela composição das ações de $M_{1}, M_{2}$ e $M_{3}$, nesta ordem.
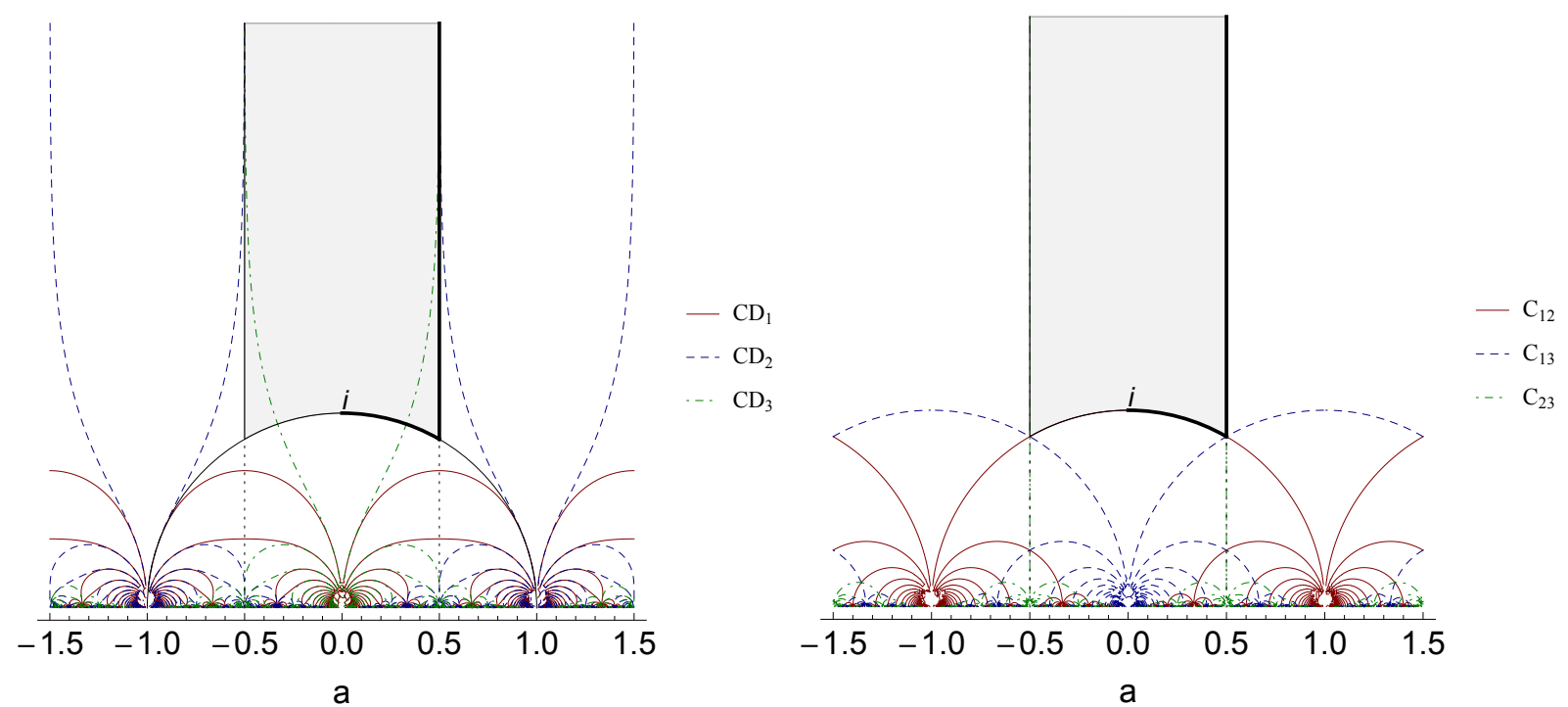

Figura 5.29: Diagramas de bifurcação dos equilíbrios (à esquerda) e das conexões (à direita), representados no dominio fundamental $\mathcal{D}$. 


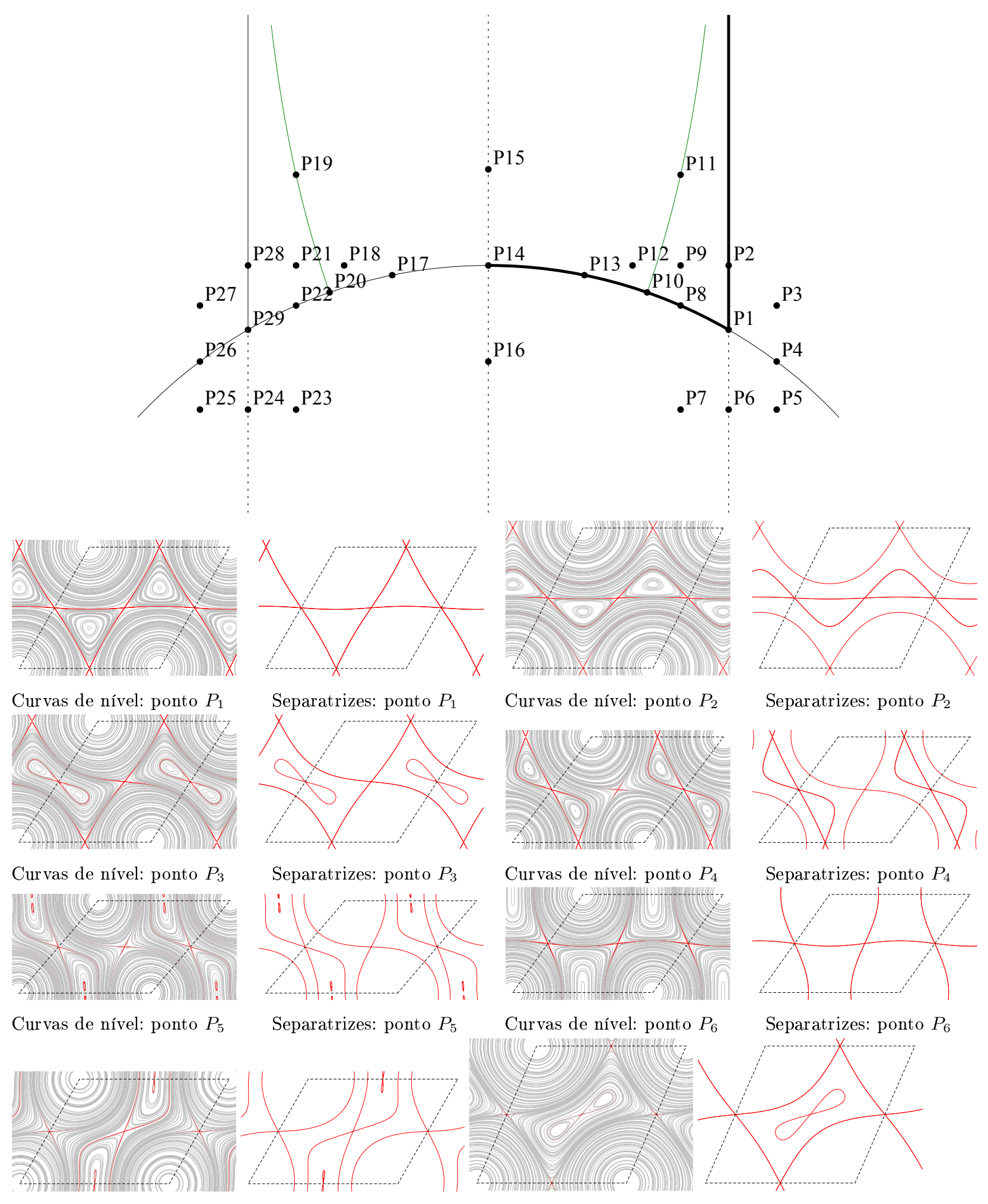

Curvas de nível: ponto $P_{7} \quad$ Separatrizes: ponto $P_{7} \quad$ Curvas de nível: ponto $P_{8} \quad$ Separatrizes: ponto $P_{8}$

Figura 5.30: Retratos de fase correspondentes aos pontos de $P_{1}$ a $P_{8}$. 


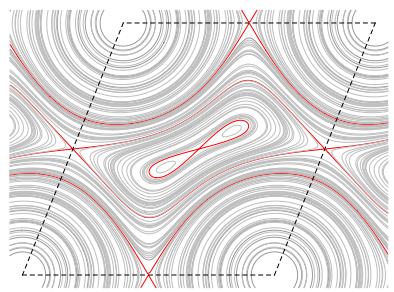

Curvas de nível: ponto $P_{9}$

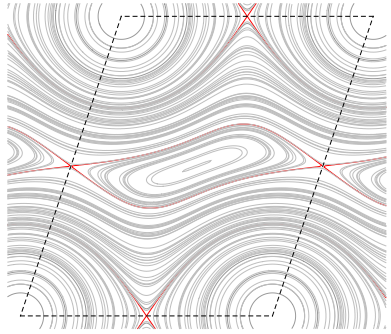

Curvas de nível: ponto $P_{11}$

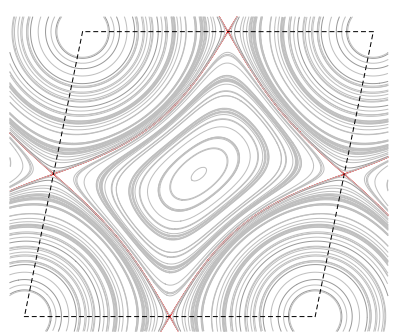

Curvas de nível: ponto $P_{13}$

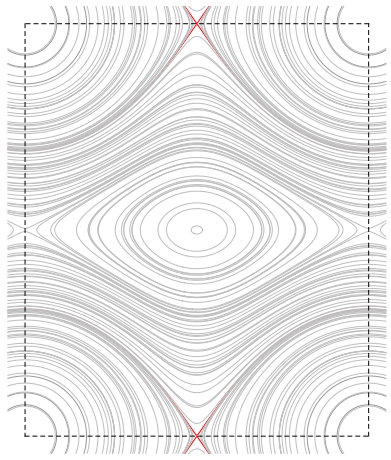

Curvas de nível: ponto $P_{15}$

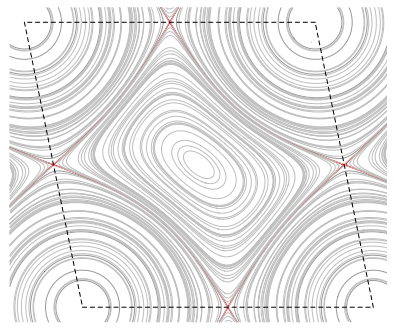

Curvas de nível: ponto $P_{17}$

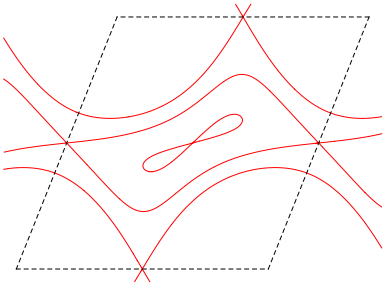

Separatrizes: ponto $P_{9}$

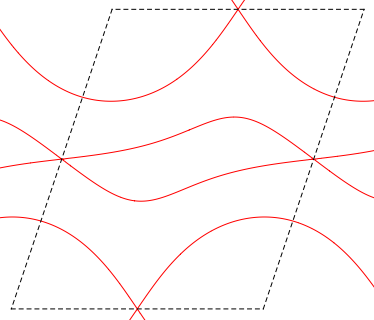

Separatrizes: ponto $P_{11}$

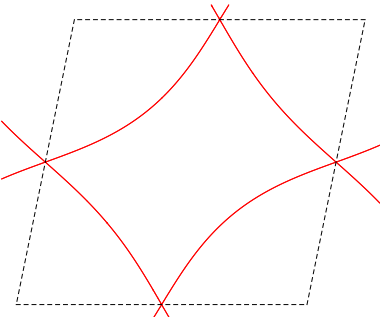

Separatrizes: ponto $P_{13}$

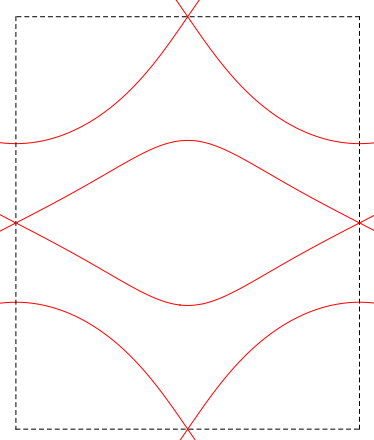

Separatrizes: ponto $P_{15}$

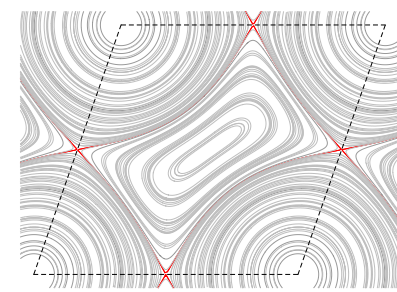

Curvas de nível: ponto $P_{10}$

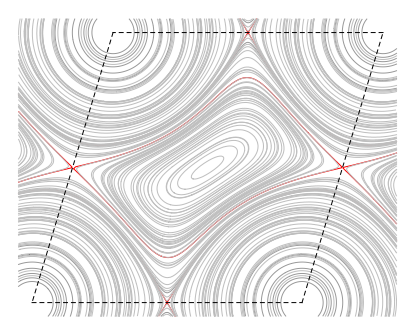

Curvas de nível: ponto $P_{12}$

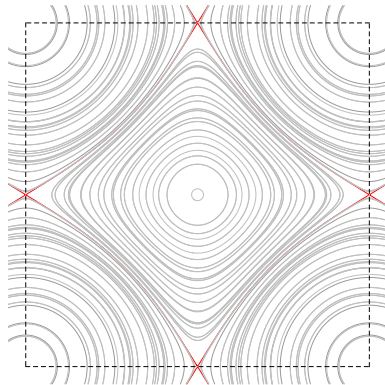

Curvas de nível: ponto $P_{14}$

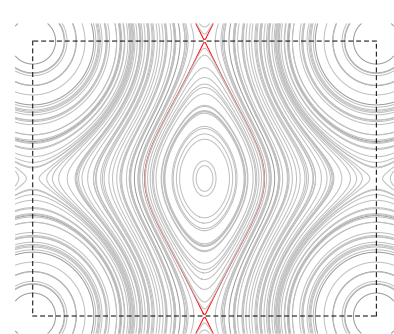

Curvas de nível: ponto $P_{16}$

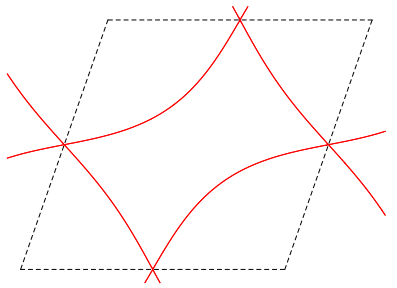

Separatrizes: ponto $P_{10}$

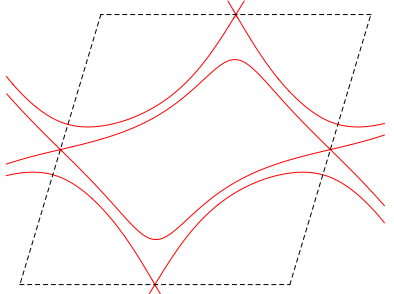

Separatrizes: ponto $P_{12}$

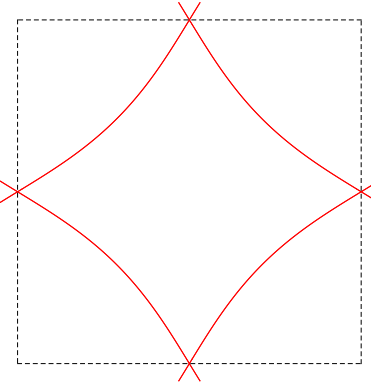

Separatrizes: ponto $P_{14}$

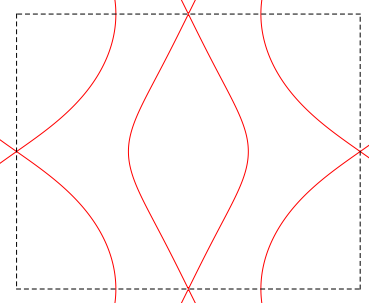

Separatrizes: ponto $P_{16}$
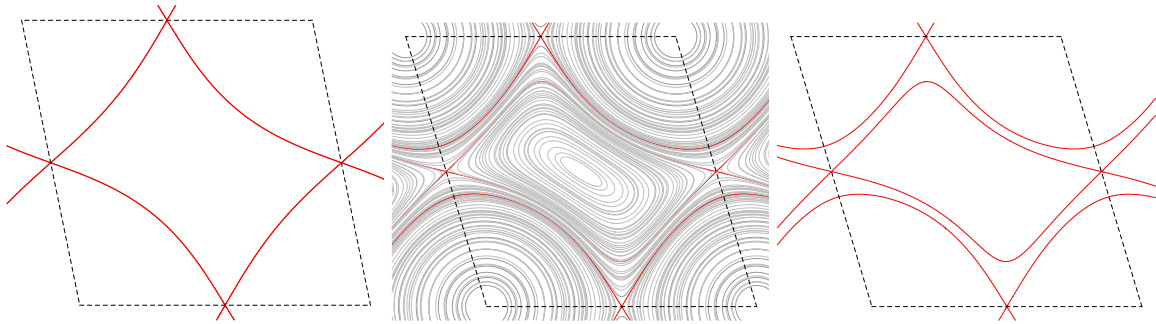

Separatrizes: ponto $P_{17} \quad$ Curvas de nível: ponto $P_{18} \quad$ Separatrizes: ponto $P_{18}$

Figura 5.31: Retratos de fase correspondentes aos pontos de $P_{9}$ a $P_{18}$. 


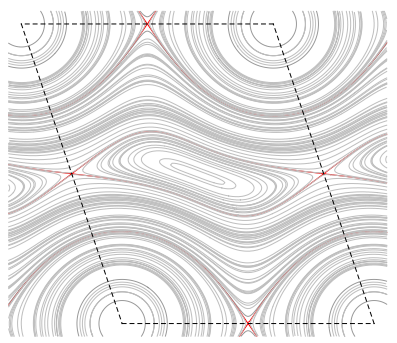

Curvas de nível: ponto $P_{19}$

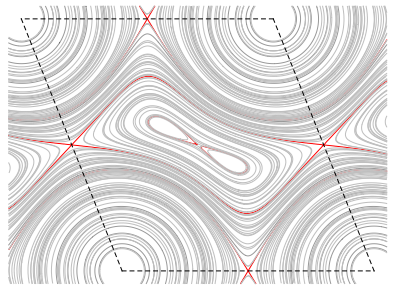

Curvas de nível: ponto $P_{21}$

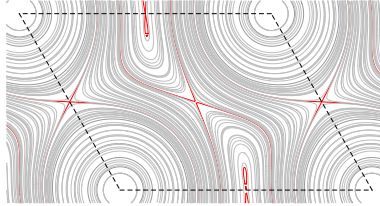

Curvas de nível: ponto $P_{23}$

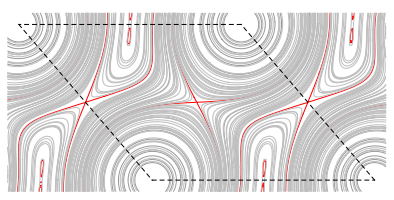

Curvas de nível: ponto $P_{25}$

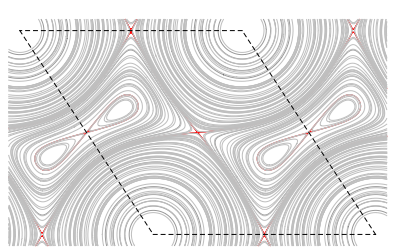

Curvas de nível: ponto $P_{27}$

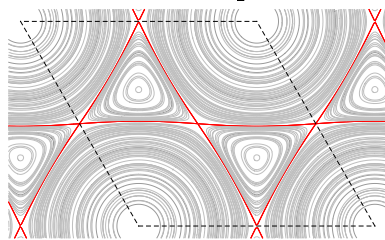

Curvas de nível: ponto $P_{29}$

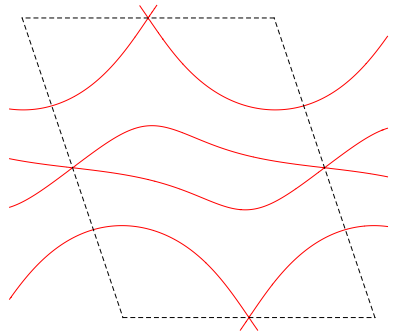

Separatrizes: ponto $P_{19}$

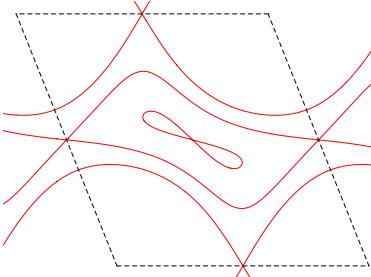

Separatrizes: ponto $P_{21}$

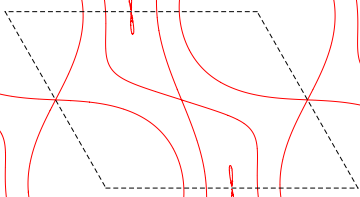

Separatrizes: ponto $P_{23}$

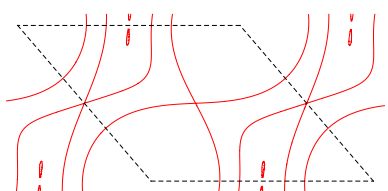

Separatrizes: ponto $P_{25}$

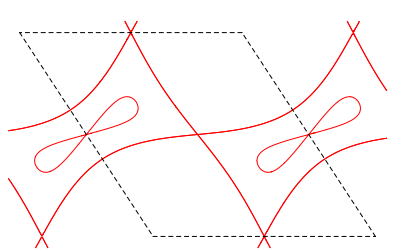

Separatrizes: ponto $P_{27}$

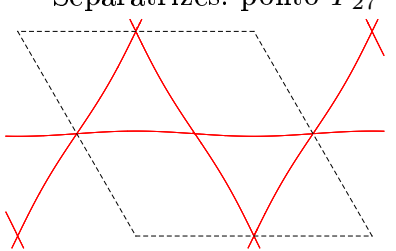

Separatrizes: ponto $P_{29}$

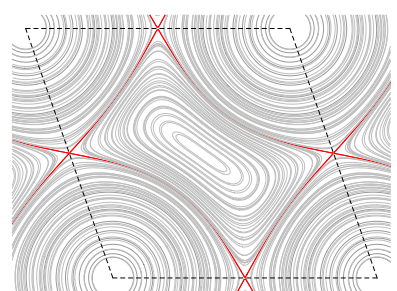

Curvas de nível: ponto $P_{20}$

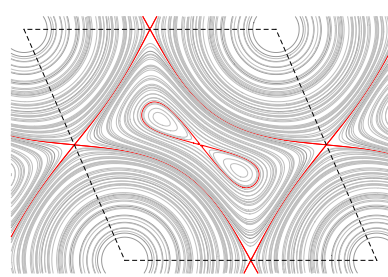

Curvas de nível: ponto $P_{22}$

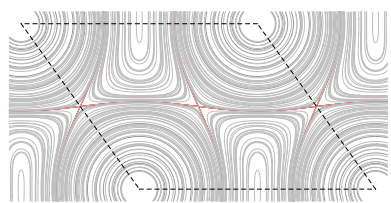

Curvas de nível: ponto $P_{24}$

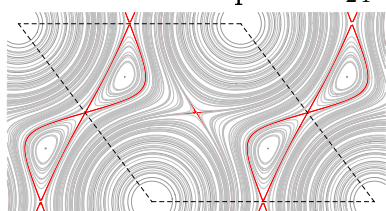

Curvas de nível: ponto $P_{26}$

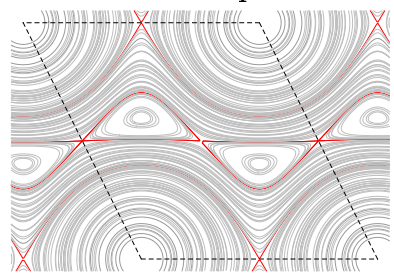

Curvas de nível: ponto $P_{28}$

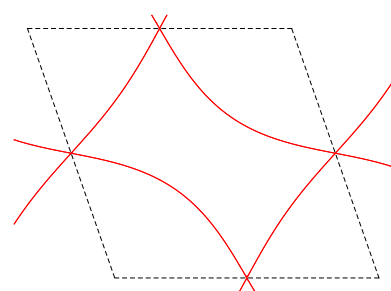

Separatrizes: ponto $P_{20}$

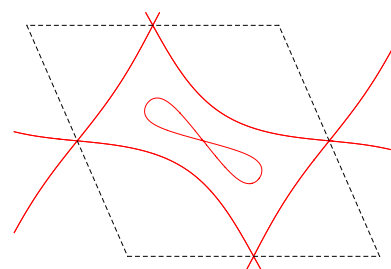

Separatrizes: ponto $P_{22}$

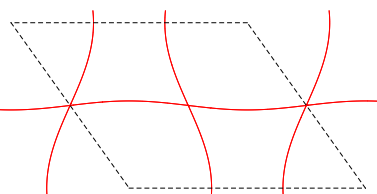

Separatrizes: ponto $P_{24}$

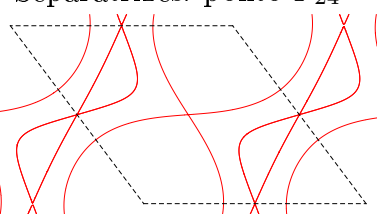

Separatrizes: ponto $P_{26}$

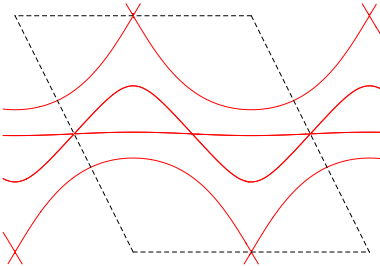

Separatrizes: ponto $P_{28}$

Figura 5.32: Retratos de fase correspondentes aos pontos de $P_{19}$ a $P_{29}$. 


\section{Observações adicionais}

Em todos os diagramas de bifurcação apresentados verificamos algumas particularidades em comum, como, por exemplo, na figura 5.9, onde nota-se que o conjunto que define o nível zero da função $F_{12}$ possui pontos de acumulação na reta $b=0$ e estes pontos não formam um conjunto discreto. Este fato pode ser verificado na figura 5.33, onde são apresentados os gráficos da função $F_{12}(\cdot, b)$ com valores de $b$ constantes próximos de zero. Os zeros destas funções são os pontos da reta $b=$ cte que intersectam o conjunto $C_{12}$. O mesmo comportamento verifica-se para os conjuntos $C_{13}, C_{23}$ e para os conjuntos $C D_{1}, C D_{2}$ e $C D_{3}$ estudados no capítulo anterior. Tal fato deve-se à perda de analiticidade das funções que definem estes conjuntos nos pontos da reta $b=0$.
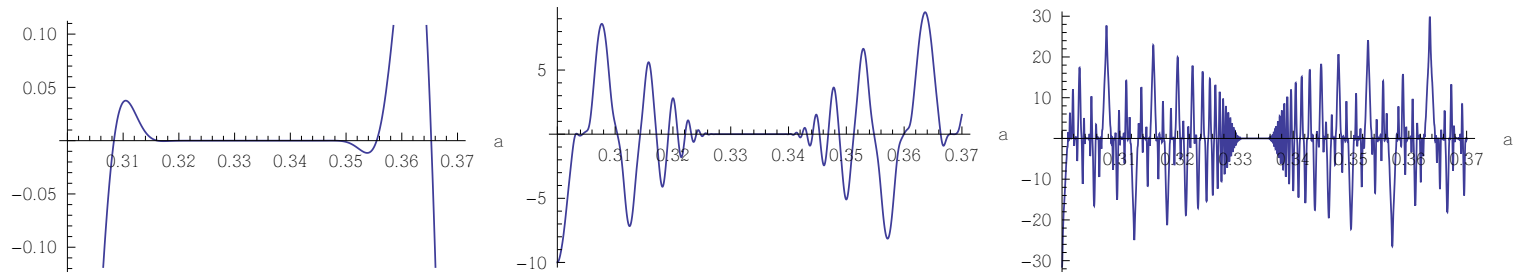

Figura 5.33: Gráfico da função $F_{12}$ em uma vizinhaça de $a=0.335$ para valores pequenos de $b$ : da esquerda para a direita, temos $b=0.01, b=0.001$ e $b=0.0001$.

Em 5.34 apresentamos uma ilustração de algumas curvas de nível da função $F_{12}$ e uma imagem ampliada de uma pequena vizinhaça do ponto $(0.36,0)$, revelando uma estrutura fractal destas curvas na proximidade da reta $b=0$.
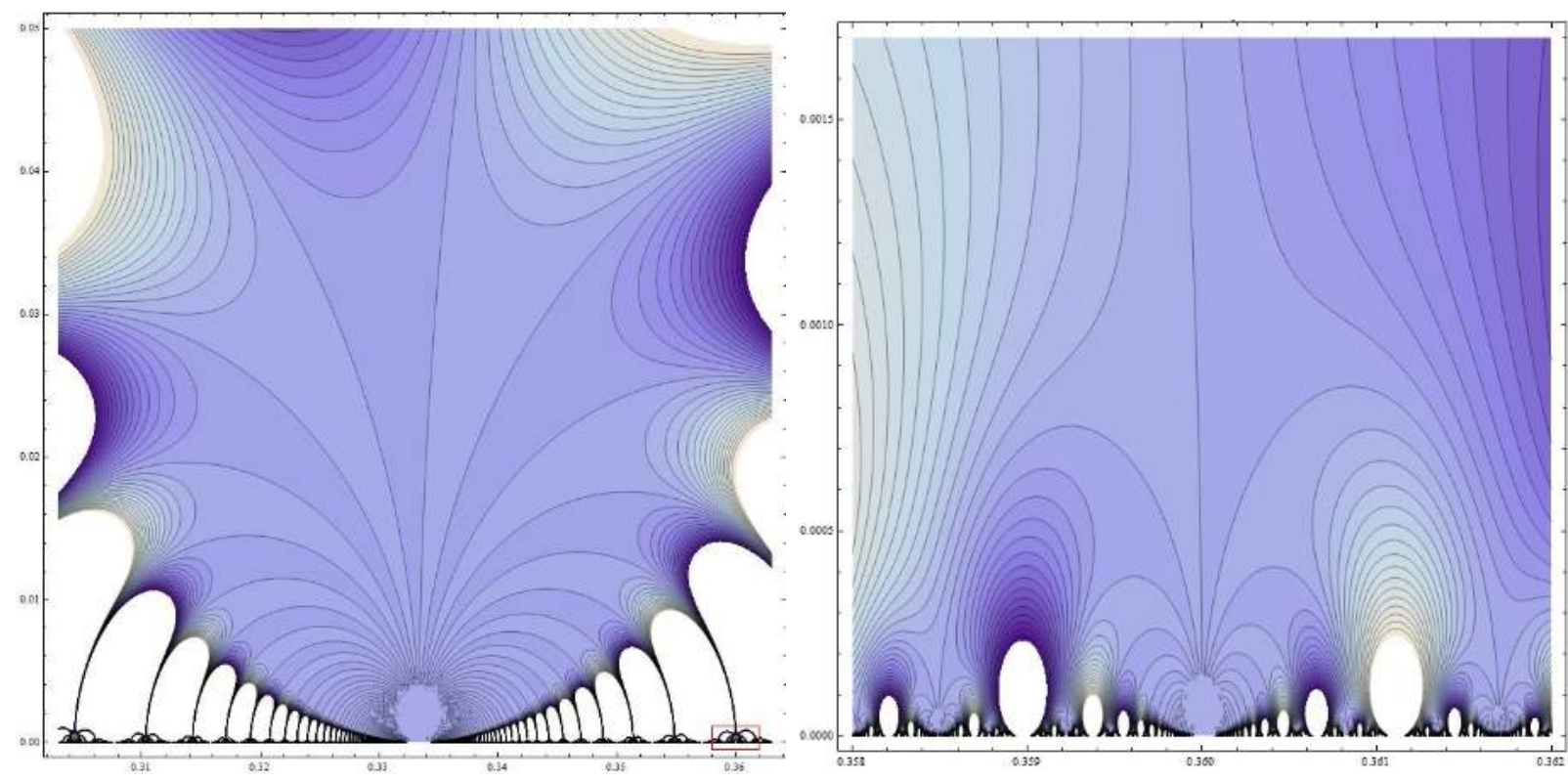

Figura 5.34: Curvas de nível da função $F_{12}$ na vizinhança de $(0.36,0)$. A figura à direita é uma ampliação da região em destaque na primeira figura. 


\section{Capítulo 6}

\section{Conclusões}

O estudo da dinâmica de vórtices em superfícies abre uma grande perspectiva de trabalho em problemas que envolvem diferentes ramos da matemática. Análise, teoria de potencial, geometria Riemanniana, superfícies de Riemann, equações diferenciais parciais, topologia e sistemas dinâmicos são algumas das áreas mais diretamente envolvidas, de modo que a busca por problemas interessantes representa um grande desafio em virtude desta exigência multidisciplinar. Considerado apenas do ponto de vista matemático, a dinâmica de vórtices consiste num sistema dinâmico bem definido cuja riqueza chama atenção não apenas por seu caracter lúdico e estético, como também pela possibilidade de estabelecer e revelar novas conexões entre problemas já conhecidos da matemática. Outro aspecto interessante da dinâmica de vórtices é sua utilidade no desenvolvimento de algorítmos e métodos numéricos que permitam obter soluções aproximadas da equação de Euler em domínios do plano, o que suscita o questionamento quanto a possibilidade de generalizar tais resultados para o caso de introduzirmos curvatura não nula no domínio do escoamento. Neste sentido, é importante analizar a relevância da curvatura na modelagem da interação entre vórtices atmosféricos e o relevo do ambiente. Experimentos recentes que exibem a evolução de vórtices em películas de sabão despertam a curiosidade natural em descobrir se a dinâmica apresentada é regida pelas equações obtidas em [Hal] e [BK08] ou se é necessário incluir novos elementos ao modelo. Em virtude da simplicidade de se realizar tais experimentos, tem-se a oportunidade de desenvolver uma boa interação entre a física e a matemática, possibilitando tanto a melhor compreensão do modelo físico como a formulação de interessantes problemas de sistemas dinâmicos e equações diferenciais parciais.

\subsection{Considerações Finais}

As hipóteses e considerações físicas adotadas na primeira parte deste trabalho nos permitiram obter uma dedução relativamente simples e direta para as equações da dinâmica de vórtices em superfícies. Dois aspectos foram cruciais para o sucesso da abordagem desenvolvida. O primeiro é a definição da força que o fluido exerce sobre um vórtice, a qual não pode ser calculada diretamente e portanto definimo-la a partir de uma sequência fracamente convergente à medida que representa o sistema de vórtices com vorticidade de fundo constante. O segundo é a validade da propriedade de localização; na verdade, basta que tenhamos uma sequência delta com vorticidade de fundo constante representando a vorticidade inicial e que seja preservada pela equação de Euler para que a dinâmica de vórtices esteja bem definida. Daí infere-se o significado da dinâmica de vórtices: suas trajetórias determinam uma aproximação para a evolução pela equação de Euler de pequenas regiões com alta concentração de vorticidade. 


\subsection{Sugestões para Pesquisas Futuras}

Com base no conteúdo desenvolvido neste trabalho, apresentamos a seguinte lista de possíveis temas para investigações futuras:

i) Considerando o exemplo do traçador passivo no disco unitário, apresentado no Capítulo 5, propomos o estudo da dinâmica do traçador com vorticidade de fundo constante e efetuando uma perturbação do bordo do disco. Comparar o resultado com aquele obtido ao perturbarmos a órbita do traçador passivo, conforme feito em [ZF93] para o caso de vorticidade de fundo nula.

ii) Estudar a dinâmica de dois vórtices no disco unitário considerando curvatura não nula e vorticidade de fundo constante. Analizar a dinâmica do traçador passivo sob as condições descritas no ítem $i$ ).

iii) Descrever a dinâmica de três vórtices no toro plano com vorticidade de fundo constante.

iv) Descrever e analizar a dinâmica de dois vórtices no toro mergulhado em $\mathbb{R}^{3}$. Considerar, inicialmente, o toro de rotação. Mais geralmente, estudar a dinâmica de dois vórtices em $\mathcal{T}(\tau)$, onde $\mathcal{T}(\tau)$ é o toro mergulhado em $\mathbb{R}^{3}$ que é conforme a $T(\tau)=\mathbb{C} / \Lambda(\tau)$.

v) Estudar a dinâmica de vórtices em superfícies mínimas.

vi) Reproduzir os experimentos que exibem a evolução de vórtices em películas de sabão e comparar com o modelo matemático na superficie mínima correspondente.

vii) Determinar condições necessárias e suficientes para que valha a propriedade de localização em domínios do plano com curvatura e vorticidade de fundo não nulas. 


\section{Referências Bibliográficas}

[Ahl79] L. V. Ahlfors. Complex Analysis. McGraw-Hill Book Company, New York, 1979. 59, 60

[AK98] V. I. Arnold e B. Khesin. Topological Methods in Hydrodynamics. Springer, New York, first edição, 1998. 2, 15

[Arn89] V. I. Arnold. Mathematical Methods of Classical Mechanics. Springer-Verlag, New York, second edição, 1989. 2

[Arn92] V. I. Arnold. Ordinary Diferential Equations. Springer-Verlag, Berlin, first edição, 1992. 2

[Aub98] T. Aubin. Some Nonlinear Problems in Riemannian Geometry. Springer-Verlag, Berlin, third edição, 1998. 2, 25, 31

[BK08] S. Boatto e J. Koiller. Vortices on closed surfaces. http://arxiv.org/pdf/0802.4313.pdf, 2008. Preprint, pp.1-16. iii, v, vii, 1, 2, 24, 25, 71

[Cro10] D. Crowdy. A new calculus for two-dimensional vortex dynamics. Theor. Comput. Fluid Dyn., 24:9-24, 2010. 2

[CS00] H. E. Cabral e D. Schmidt. Stability or relative equilibria in the problem of $\mathrm{n}+1$ vortices. SIAM Journal on Mathematical Analysis, 31:231-250, 2000. 2

[dC88] M. do Carmo. Riemannian Geometry. BirkhÃuser, New York, second edição, 1988. 2, 16

[FG97] M. Flucher e B. Gustafsson. Vortex motion in two-dimensional hydrodynamics. Relatório Técnico TRITA-MAT-1997-MA-02, Deparment of Mathematics, KTH - Stockholm, Sweden, Stockholm, Sweden, 1997. v, 1, 2, 15, 16, 24, 30

[FK92] H. M. Farkas e I. Kra. Riemann Surfaces. Springer-Verlag, New York, second edição, 1992. 2

[Fri66] K. O. Friedrichs. Special Topics in Fluid Dynamics. Notes on Mathematics \& Its Applications, New York, 1966. 1, 2, 8

[Gus79] B. Gustafsson. On the motion of a vortex in two-dimensional flow of an ideal fluid in simply and multiply connected domains. Relatório Técnico TRITA-MAT-1979-7, Deparment of Mathematics, KTH - Stockholm, Sweden, Stockholm, Sweden, 1979. v, 2

[Hal] D. Hally. Stability of streets of vortices on surfaces of revolution with a reflection symmetry. J. Math. Phys., 21(1). v, vii, 1, 2, 22, 71

[IT92] Y. Imayoshi e M. Taniguchi. An Introduction to Teichmuller Spaces. Springer-Verlag, Tokyo, first edição, 1992. 61, 62

[Kle63] F. Klein. On Riemann's Theory of Algebraic Functions and their Integrals. Dover Publications, Inc., New York, first edição, 1963. 2 
[Lin41a] C. C. Lin. On the motion of vortices in two dimensions - i. existence of the kirchhoff-routh function. Proc Natl Acad Sci U S A., 27(12):570-575, 1941. 1, 31

[Lin41b] C. C. Lin. On the motion of vortices in two dimensions-ii some further investigations on the kirchhoff-routh function. Proc Natl Acad Sci U S A., 27(12):575-577, 1941. 1, 31

[LM09] C. Lacave e E. Miot. Uniqueness for the vortex-wave system when the vorticity is initially constant near the point vortex. SIAM J. Math. Anal., 41:1138-1163, 2009. 34

[LW10] C-S Lin e C-L Wang. Elliptic functions, green functions and the mean field equations on tori. Annals of Mathematics, 172:911-954, 2010. 2, 43, 44, 45

[MB02] A. J. Majda e A. L. Bertozzi. Vorticity and Incompressible Flow. Cambridge University Press, Cambridge - UK, first edição, 2002. 2, 5, 32

[MP82] C. Marchioro e M. Pulvirenti. Hydrodynamics in two dimensions and vortex theory. Communications in Mathematical Physics, 84:483-503, 1982. 2

[MP93] C. Marchioro e M. Pulvirenti. Vortices and localization in euler flows. Communications in Mathematical Physics, 154:40-61, 1993. v, vii, 2, 12, 29, 32, 33

[MP94] C. Marchioro e M. Pulvirenti. Mathematical Theory of Incompressible Nonviscous Fluids. Springer-Verlag, New York, 1994. 1, 2, 5

[New01] P. Newton. The N-vortex problem: analytical techniques. Springer-Verlag, New York, first edição, 2001. 2, 22, 24

[PL87] LF Tam P. Li. Symmetric green's functions on complete manifolds. American Journal of Mathematics, 109(6):1129-1154, 1987. 2, 31

[SA00] M. A. Stremler e H. Aref. Motion of three point vortices in a periodic parallelogram. Journal of Fluid Mechanics, 392:101-128, 2000. 27, 42, 45

[Sot79] J. Sotomayor. Licoes de Equacoes Diferenciais Ordinarias. IMPA Projeto Euclides, Rio de Janeiro, first edição, 1979. 2

[Ste05] J. Steiner. A geometrical mass and its extremal properties for metrics on $s^{2}$. Duke Math. J., 129(1):63-86, 2005. 31, 43

[Str88] D. J. Struik. Lectures on Classical Differential Geometry. Dover Publications, Inc., New York, first edição, 1988. 2, 20

[Str10] M. A. Stremler. On relative equilibria and integrable dynamics of point vortices in periodic domains. Theor. Comput. Fluid Dyn., 24:25-37, 2010. 42

[ZF93] L. Zannetti e P. Franzese. Advection by a point vortex in closed domains. Eur. J. Mech., B/Fluids, 12:43-67, 1993. 37, 40, 72 


\section{Índice Remissivo}

Bernoulli

segunda lei de, 7

bifurcação

curvas de nível da função de Green no toro, 44

dinâmica de vórtices

abordagem alternativa, 11

abordagem de Friedrichs, 5

condições iniciais e de contorno, 30

formulação hamiltoniana, 27

disco unitário, 37

função de corrente, 38

traçador passivo, 40

equação de Euler, 5

PVIEE, 31

fluido

irrotacional, 6

força, 5

sobre objeto estático, 6

sobre um vórtice, $7,8,13$

sobre uma partícula material, 11

função

de corrente, 6

função de Green

toro, 43

Hally

equações de, 22

Helmholtz-Kirchhoff

equações de, 9

J.J. Thompson, 1

Kirchhoff, 1

localização

propriedade, 31, 33

Lord Kelvin, 1

marcação, 62

moduli

espaço, 61 onda-vórtice

sistema, 33

potencial

complexo, 7,14

de velocidade, 7

projeção, 42

propriedade de localização, 12

reticulado, 42

base, 59

geradores, 42

Routh

regra, 24

Teichmüler

espaço, 62

toro, 42

função de Green, 43

marcado, 62

toro plano, 42

função de corrente, 42

unimodular

transformação, 59

vórtice

com massa, 9

definição, 1

em coordenadas isotérmicas, 15

em superfícies fechadas, 25

em um domínio do plano, 12

força sobre um, 13

friedrichs, 6

livre, 9

vorticidade, 6

em superfícies, 15

W. Thomson, 1 